\title{
Sex is a defining feature of neuroimaging phenotypes in major brain disorders
}

\author{
*Lauren E. Salminen ${ }^{1},{ }^{*}$ Meral A. Tubi ${ }^{1}$, Joanna Bright ${ }^{1}$, Sophia I. Thomopoulos ${ }^{1}$, \\ Alyssa Wieand ${ }^{1}$, Paul M. Thompson ${ }^{1}$ \\ ${ }^{1}$ Imaging Genetics Center, Mark and Mary Stevens Neuroimaging \& Informatics Institute, \\ Keck School of Medicine of USC, Marina del Rey, CA, USA \\ *Denotes equal contribution
}

\section{Acknowledgments:}

This work was supported by National Institutes of Health (NIH) grant numbers P01 AG055367, P41 EB015922, R01 AG060610, and R01 MH116147 (to P.M.T.), T32 MH111360 (Levitt), and F31 AG059356 (to M.A.T.). The ENIGMA Consortium is supported in part by NIH grant U54 EB020403 from the Big Data to Knowledge (BD2K) Program, and NIA grant R01 AG058854

(to P.M.T.).

\section{Disclosures and Conflicts of Interest}

PMT received a research grant from Biogen, Inc. (Boston, USA) and served as a consultant for Kairos Venture Capital, Inc., on topics unrelated to this manuscript. The other authors report no conflicts of interest.

\section{Data Availability Statement}

Data sharing is not applicable to this article as no new data were created or analyzed in this study. 


\begin{abstract}
Sex is a biological variable that contributes to various elements of brain and behavior in clinical and non-clinical samples. In clinical samples, several studies show sex differences in disease risk, prevalence, and expression of clinical symptoms, yet sex differences in MRI outcomes are still understudied. Here we review the existing literature on sex differences in brain structure in normative samples and in 14 distinct psychiatric and neurological disorders. We discuss commonalities and sources of variance in study designs, analysis procedures, disease subtype effects, and the impact of these factors on MRI interpretation. Lastly, we identify key problems in the neuroimaging literature on sex differences and offer potential recommendations to address current barriers and optimize rigor and reproducibility. In particular, we emphasize the importance of largescale neuroimaging initiatives such as the Enhancing Neurolmaging Genetics through MetaAnalyses (ENIGMA) consortium, the UK Biobank, Human Connectome Project, and others to provide unprecedented power to evaluate sex-specific phenotypes in major brain diseases.
\end{abstract}

Abbreviated Abstract (2-3 sentences): Here we review the human neuroimaging literature on sex differences in normative samples and in 14 major brain disease. We identify several key barriers to advancing scientific knowledge in sex effects on neuroimaging and offer recommendations to mitigate these challenges, particularly through large-scale neuroimaging.

Keywords: sex, gender, male, female, psychiatry, neurology, neuroimaging, structural MRI, diffusion MRI, ENIGMA 


\section{Introduction}

For many decades, the majority of biomedical knowledge was based on studies of males, leading to major disparities in our understanding of disease etiology, symptom presentation, treatment strategy, and clinical response in females. In 1993, the National Institutes of Health (NIH) in the U.S. implemented the Revitalization Act (https://orwh.od.nih.gov/resources/pdf/NIHRevitalization-Act-1993.pdf), which mandated that females must be included in $\mathrm{NIH}$-funded clinical trials. Unfortunately, sex biases in findings from basic and pre-clinical research persisted in both human and animal studies. A 2009 study of sex biases in animal research revealed that $80 \%$ of all animal studies examined male rodents only, across 8 different scientific fields [Beery and Zucker, 2011], with the strongest male biases in neuroscience and pharmacology. Male animals still dominate the biomedical animal literature, particularly in cardiovascular research - a field with known sex differences in health risks, symptom presentation, and treatment response [Ramirez et al., 2017].

Sex disparities in human biomedical research have begun to be addressed in recent years, but they are still understudied. A recent bibliometric analysis of over 11 million papers [Sugimoto et al., 2019] outlined the severity of sex biases across various fields of medicine, with the vast majority of studies neglecting to report sex characteristics of the sample. Of the disciplines studied, psychiatric and neurological studies reported sex in approximately $80 \%$ and $65 \%$ of research papers, respectively. The poorest sex reporting came from pharmacology studies, where only $24 \%$ of papers disclosed sex characteristics. More disturbingly, greater sex reporting was found in publications in lower impact journals over time, meaning lower visibility for papers that may adequately address sex effects [Sugimoto et al., 2019]. To address these persisting biases, the NIH mandated that all grant proposals must address sex as a biological variable (NOT-OD-15-102, http://grants.nih.gov/grants/guide/notice-files/NOT-OD-15-102.html), even for basic and pre-clinical research. Specifically, investigators need to provide a detailed plan to analyze equal numbers of males and females, or provide sufficient justification if sex 
distributions would be unequal [Clayton, 2018]. Early reports suggest this mandate has so far improved sex reporting and awareness of its importance in clinical and preclinical studies [Lee, 2018; Zucker and Beery, 2019]. Even so, decades of biased work still dominate the literature and continue to be published [Mamlouk et al., 2020].

Recently, Mamlouk et al. [Mamlouk et al., 2020] conducted a systematic review of 1,827 neuroscience papers (25\% of them human studies) published in six high impact journals in 2017 - one year after the NIH mandate to address sex as a biological variable. Of the papers reviewed, approximately $44 \%$ reported that there were both males and females in the study, but sex was only considered an experimental variable in $8 \%$ of studies. There was a stark difference in the proportions of single sex studies ( $26 \%$ male-only vs. $5 \%$ female-only), with $16 \%$ of studies not reporting sex. Further, human neuroscience studies were the least likely to include sex as an experimental variable compared to other research models ( $75 \%$ of published human studies compared to $49 \%$ and $22 \%$ of studies in mice and rats, respectively). This number is alarming as the term "experimental variable" was operationally defined using broad criteria (e.g., any form of statistical comparison between males and females, and/or the use of sex as a covariate). Although Mamlouk et al. [Mamlouk et al., 2020] did note decreasing sex biases over time, the degree of bias change depended significantly on the research model used, and the publishing journal. Interestingly, sex reporting did not differ significantly between studies with and without $\mathrm{NIH}$ funding (57\% were $\mathrm{NIH}-$ funded).

Previous preclinical studies justified sex imbalances by claiming that females introduced too much variability into research designs due to hormonal fluctuations along the oestrous cycle [Sugimoto et al., 2019][Wang, 1923; Zucker and Beery, 2010]. However, there is considerable evidence from animal studies showing that variability in behavioral, biological, and molecular end points is consistent between females and males [Sugimoto et al., 2019]. Further, a meta-analysis of 293 rodent studies revealed greater variability in males than females on indices of hormones, metabolism, and morphological traits [Prendergast et al., 2014]. 
The female reproductive cycle also has been used to justify explicit recruitment biases against women in clinical research. For example, pregnant women were considered a vulnerable population that was broadly "protected" (i.e., excluded) from clinical research until the U.S. Department of Health and Human Services (HHS) removed pregnant women as a vulnerable category in a 2018 amendment to the Federal Policy for the Protection of Human Subjects ('Common Rule') https://www.hhs.gov/ohrp/regulations-and-policy/regulations/45-cfr46/index.html [Biggio, 2020]. The amended policy (implemented in 2019) was a response to the US Task Force on Research Specific to Pregnant Women and Lactating Women, which stated that pregnant women are fully capable of making medical decisions for themselves and their fetus, and that the term "vulnerable" restrained the right to individual autonomy [Costantine et al., 2020; Heyrana et al., 2018]. Further, the exclusion of pregnant women from clinical research led to gross knowledge gaps related to treatments and interventions that can be safely administered during pregnancy. It is too soon to tell whether the new amendment will reduce biases against pregnant women in clinical research; evidence from the emerging COVID-19 literature suggests that explicit sex biases in recruitment are still in play [Costantine et al., 2020]. Finally, implicit sex biases are also well-documented in healthcare and clinical research and contribute to lower participation of women in clinical trials [Chadwick and Baruah, 2020; Chapman et al., 2013; Daugherty et al., 2017; Hansen et al., 2019; Salles et al., 2019].

This review is designed to illuminate these gaps in the human neuroimaging literature on sex differences in brain diseases, including common methods and design strategies that miss opportunities to sufficiently address the role of sex in brain health and disease. To provide mechanistic context for the purported sex differences in various brain disorders and conditions, we first provide a brief overview of the function and trajectory of the primary sex hormones across the lifespan and their associations with neuroimaging indices. We then discuss normative sex differences in brain structure and function from population-based samples, as these differences are preserved in many clinical conditions and should not be interpreted as an outcome of disease. 
We chose to review both psychiatric and neurodegenerative diseases, as many psychiatric conditions are risk factors for neurodegenerative diseases and dementia later in life [Ahearn et al., 2020; Almeida et al., 2017; Diniz et al., 2017; Gimson et al., 2018; Kørner et al., 2009; Kuring et al., 2020; Mrabet Khiari et al., 2011; Ribe et al., 2015; Singh-Manoux et al., 2017; Truelsen et al., 2002; Yaffe et al., 2010; Zilkens et al., 2014], and are associated with abnormalities in similar neuroimaging measures. Psychiatric disorders typically have an earlier age of onset than neurodegenerative conditions, with peak prevalences and symptom severities during the critical decades (ages 20-40) when neurodegenerative pathologies are seeded [Jones, 2013; Kessler et al., 2007; Zilkens et al., 2014]. Thus, understanding the role of sex on psychiatric neuroimaging phenotypes may inform etiologic mechanisms of neurodegenerative disease and dementia, and help in the development of novel intervention strategies.

Our literature review was conducted using a combination of search terms in PubMed, Google Scholar, and bioRxiv. Search terms to query information about sex included "sex", "gender", "males”, "females", "men”, and "women" in conjunction with comparison terms such as "differences", "biases", "disparities", and "confounds". Additional phrases included "sex-specific", "sex-by-age interaction", "sex-by-diagnosis interaction", "sex covariate", "adjusting for sex", "nuisance covariate", "sexually dimorphic", "biological sex", and "genetic sex". We prioritized the most recent publications first and gradually expanded our search in two-year increments; all years were searched if terms yielded no hits in the last decade. As this is not a systematic review and our paper covers 14 distinct neurological and psychiatric conditions (in addition to normative studies), it was not possible to account for every published MRI study on sex effects or every imaging modality within these published studies. Instead, we prioritized large-scale studies from biobanks (e.g., UK Biobank), consortia (e.g., ENIGMA), and systematic reviews that focused on traditional structural and diffusion MRI outcomes when available. Small-scale studies were included when large studies examining specific sex effects had not been conducted, were inconclusive, or contradicted other work. For conditions with a considerable literature on sex 
effects on neuroimaging (e.g., multiple sclerosis, Alzheimer's disease), we prioritized studies that contributed to key themes or findings related to sex and neuroimaging. A summary of the studies reviewed is provided in Table $\mathbf{S 1}$ in the Supplementary Material. Finally, many of the conditions discussed in this review may have developmental origins in childhood and adolescence. This literature is highly complex and has been reviewed in detail in other work [Deak et al., 2015; Earls, 1987; Schwarz and Bilbo, 2012]. As such, we elected to focus on sex differences in the adult brain to adhere to page constraints and avoid an overly exhaustive and redundant review.

\section{Role of primary sex hormones in the brain}

\section{Developmental influences and lifespan trajectories}

In humans, the impact of sex differences on health and disease begins as early as 50 days post-conception when sex is determined through a cascade of genetic interactions beginning with the sex-determining region of the $Y$ chromosome (SRY gene, chromosome 9) [Mamsen et al., 2017]. When SRY transcripts are present, they initiate sexual differentiation of bipotential gonads by activating the SRY-box 9 gene (SOX9) approximately 50 days post-conception [Mamsen et al., 2017]. SOX9 regulates the transcription of anti-mullerian hormone along with other male-specific genes that promote androgen biosynthesis and the development of male sex organs. In the absence of $S R Y$, female reproductive genes (WNT4, RSPO-1, FOXL2) promote the development of ovaries and inhibit differentiation of the testis, resulting in estrogen and progesterone synthesis. The divergent mechanisms of primary sex hormones are enacted, in part, by the location of hormone synthesis and the function and regional distribution of target receptors. Biosynthesis of sex steroids primarily occurs in male and female reproductive organs, but they are also synthesized in the brain and other tissues de novo from cholesterol [Hu et al., 2010]. Many additional genetic and epigenetic factors have been identified in sex differentiating 
pathways, as detailed elsewhere [Mamsen et al., 2017; Rotgers et al., 2018].

Sex hormones are believed to have organizational (permanent) and activational (dynamic) effects that impact disease manifestation, timing, and neuropathological progression, with associated changes in brain structure and function [Herting and Sowell, 2017; Schulz and Sisk, 2016]. Organizational effects of sex hormones are "permanent" effects related to sexual differentation and development that occur during the perinatal period [Arnold and Breedlove, 1985] and puberty/adolescence [Schulz et al., 2009; Schulz and Sisk, 2016]. During these periods, sex hormones are believed to impart lasting effects on brain structure through complex gene-biology interactions that influence dendritic spine growth, synaptogenesis, synaptic patterning, and pruning [Arnold and Breedlove, 1985; Herting and Sowell, 2017; McCarthy, 2008; Schulz et al., 2009; Schulz and Sisk, 2016]. These organizational effects are implicated as underlying mechanisms of the "developmental origins" hypothesis- a widely accepted theory linking early life experiences to adult disease [McCarthy et al., 2012]. By contrast, activational effects of sex hormones refer to transient and dynamic effects of sex hormones that occur throughout life after neural circuits have been organized, typically during adulthood [Herting and Sowell, 2017; Schulz and Sisk, 2016].

The developmental trajectory of primary sex hormones is dynamic in both males and females. In males, testosterone levels rise during the perinatal period, reaching peak levels 1-3 months after birth. Afterwards, testosterone levels decline sharply until they plateau around 7-12 postnatal months; levels sharply rise again at puberty and plateau again around age 17 [Forest et al., 1974; Johannsen et al., 2018; Mason et al., 2020; Senefeld et al., 2020; Tomlinson et al., 2004]. Estrogen levels in males remain low in early childhood and then modestly rise around age 8 until they peak between ages $16-18$ years [Frederiksen et al., 2020]. In females, estrogen levels rise shortly after birth until approximately age 1 , when estrogen plateaus until puberty ( age 10). During puberty, estrogen levels increase until ages 15-16 [Bidlingmaier et al., 1973; Frederiksen et al., 2020]. Testosterone levels in females remain low throughout infancy and childhood, and 
modestly increase around age 6 until around age 14 [Søeborg et al., 2014]. At the onset of puberty, females experience monthly fluctuations in estrogen and progesterone according to the menstrual cycle, as detailed in the sections below. Deviations from these normal sex-specific hormone trajectories can permanently alter structural brain development and increase vulnerability to disease acutely and many years later [McCarthy, 2008; Pike, 2017].

Testosterone levels remain fairly stable throughout adulthood in both males and females [Handelsman et al., 2016], with modest decline in males that begins around the fifth decade [Feldman et al., 2002; Harman et al., 2001]. Testosterone levels are also higher in males than females throughout the lifespan [Rothman et al., 2011]. Females experience more dynamic changes in primary sex hormones (estrogen, progesterone) during adulthood due to menstruation, pregnancy, and menopause [Del Río et al., 2018]. Estrogen levels are higher in premenopausal females than males, but estrogen levels between males and females become similar after females experience menopause [Handelsman et al., 2016; Nugent et al., 2012; Rothman et al., 2011].

\section{Testosterone}

Testosterone is known for promoting muscle and bone growth, healthy libido, mood, and social behaviors such as aggression, competitiveness, and risk taking [Campbell et al., 2010; Casto and Edwards, 2016; Eisenegger et al., 2011; Tyagi et al., 2017; Walther et al., 2019]. The effects of testosterone on brain and behavior occur by binding to androgen receptors (ARs) in the forebrain, midbrain, and brainstem, with the highest concentrations in the ventromedial hypothalamus (VMH), medial preoptic area (MPOA), nucleus accumbens, basal nucleus of the stria terminalis (BNST), and septum [Davey and Grossmann, 2016]. Neuroimaging investigations relating testosterone levels to brain structure are limited in healthy adults, but suggest a link between circulating testosterone and frontal-temporal brain integrity. Specifically, a structural MRI study of healthy young adults $\left(\mathrm{N}=34,50 \%\right.$ female; ages $21.3-47.1$ years, $\mathrm{M}_{\mathrm{age}}=26.6 \pm 5.0$ years $)$ 
reported a negative association between testosterone levels and gray matter volume in the left inferior frontal gyrus (IFG) after adjusting for sex and total gray matter volume [Witte et al., 2010]. However, testosterone only explained $2.2 \%$ of total model variance compared to $32 \%$ and $47.2 \%$ explained by sex and gray matter volume, respectively. Sex-stratified analyses did not show significant associations between testosterone and gray matter volumes, likely due to low statistical power from the small sample size and limited explanatory effect of testosterone on gray matter volume in the whole sample. More recently, a study of hippocampal volume in the Vietnam Era Twin Study of Aging (VETSA) cohort ( $\mathrm{N}=445$ males, ages 51-60 years) showed that effects of salivary free testosterone (unbound to a receptor) on hippocampal volume differed based on a person's cortisol levels [Panizzon et al., 2018]. Specifically, Panizzon et al. [Panizzon et al., 2018] found that the effect of free testosterone on hippocampal volume was only significant when cortisol levels were $>1$ SD above or below the mean, such that hippocampal volumes were larger in individuals with high testosterone and high cortisol, but smaller in individuals with low testosterone and low cortisol. These associations were observed after covarying for age, ethnicity, twin pair, current alcohol use, depression, smoking status, and a history of cardiovascular disease, hypertension, and diabetes, and after correcting for lack of independence in the sample (i.e., twin clustering) [Panizzon et al., 2018].

\section{Estrogen and progesterone}

Estrogen and progesterone are important opposing sex hormones that fluctuate significantly across the female menstrual cycle. Estrogen impacts a wide range of positive biological functions beyond the reproductive system, including maintenance of bone mineral density, regulation of antioxidant defense systems and mitochondrial oxidation, maintenance of blood vessel structure and vascular tone, and enhanced neuron survival [Prabhushankar et al., 2014; Ventura-Clapier et al., 2019]. At the same time, estrogen is associated with increased production of the stress hormone, cortisol, upregulation of excitatory neurotransmitters 
(acetylcholine, dopamine) and downregulation of the inhibitory neurotransmitter, GABA [Barth et al., 2015]. These latter biological effects of estrogen increase vulnerability to adverse psychosomatic symptoms under certain conditions.

Estrogen exerts its effects by binding to G-coupled receptors that activate second messenger systems and to intracellular estrogen receptor alpha (ER- $\alpha)$ and beta (ER- $\beta$ ) to modulate transcription [Fuentes and Silveyra, 2019]. ER- $\alpha$ and ER- $\beta$ respectively modulate the excitatory and inhibitory effects of estrogen, and both receptors are located throughout the limbic system, midbrain, and brainstem to regulate the stress response in the preoptic area, arcuate and lateral habenula, periaqueductal gray, locus coeruleus, and in nuclei of the amygdala, hypothalamus, pons, and medulla oblongata [Weiser et al., 2008]. However, only ER-a is found in the ventromedial nucleus of the hypothalamus and subfornical organ, whereas only ER- $\beta$ is found in the olfactory bulb, zona incerta of the subthalamus, ventral tegmental area, cerebellum, pineal gland, and hypothalamic nuclei of the supraoptic (SON), paraventricular, suprachiasmatic, and tuberal areas [Weiser et al., 2008]. The distinct distribution of ER- $\alpha$ and ER- $\beta$ in these brain regions allows for separate interactions with neurotransmitter systems to facilitate target functions.

Progesterone is a derivative of the hormone, pregnenolone, in both males and females. Although it is most commonly known for its role in female physiology, progesterone also facilitates sperm capacitation, fertilization and immunosuppression in both sexes [Maybin and Critchley, 2011; Oettel and Mukhopadhyay, 2004]. In the brain, progesterone helps to maintain the structural integrity of myelin and regulates synaptogenesis, neuron survival and dendritic growth [Schumacher et al., 2012]. Neural functions of progesterone occur primarily through membraneassociated progesterone receptors in various brain regions including the hippocampus, amygdala, olfactory bulb, cortex, cerebellum, locus coeruleus, hypothalamus, thalamus, basal ganglia, and brainstem [Schumacher et al., 2012].

Estrogen and progesterone levels fluctuate throughout the female menstrual cycle, and 
these fluctuations have been associated with changes in mood, concentration, somatic sensations, and brain structure [Catenaccio et al., 2016]. The follicular phase and luteal phase are the two primary phases of the menstrual cycle. Menstruation generally occurs between days 1-8 of the follicular phase, during which estrogen rises until reaching peak levels at the start of the peri-ovulatory period of the follicular phase ( days 11-12). Ovulation, which typically occurs around day 14 of the menstrual cycle, marks the transition from the follicular phase to the luteal phase, and a significant drop in estrogen levels. Simultaneously, progesterone levels begin to rise during the luteal phase (progesterone levels are low during follicular phase), peaking at the start of the pre-menstrual period ( days 21-22) and declining rapidly thereafter [Catenaccio et al., 2016; Maybin and Critchley, 2011]. A recent review of 25 neuroimaging studies $(\mathrm{N}=1,321)$ [Catenaccio et al., 2016] described the brain signatures that corresponded to these phases. Four studies reported larger volumes during the follicular phase than the luteal phase, with the most consistent effects in the hippocampus, parahippocampal and mid frontal gyri. Five studies reported larger volumes during the luteal phase than the follicular phase using a mix of voxelbased morphometry (VBM) and region of interest (ROI) methods, but none reported effects in consistent regions. Three studies reported no volume differences between follicular and luteal phases. A few studies compared neuroimaging metrics (VBM, volumetry, ROI-based) between the menstrual and peri-ovulatory periods, but none reported consistent effects in any regions [Catenaccio et al., 2016]. It is worth noting, however, that the sample sizes of each of the 25 reviewed studies was small (largest $\mathrm{N}=128$, mean $\mathrm{N} \sim 32$ ) and effect sizes were not reported in any systematic way.

\section{Exogenous sources of estrogen and progesterone}

Studies of hormone contraceptive use provide naturalistic designs to understand relationships between hormonal fluctuations and brain health. These studies are important as approximately $25 \%$ of pre-menopausal females use oral hormone contraceptives or long-acting 
reversible contraceptives [Daniels and Abma, 2020]. There is increasing recognition that hormone contraceptives can influence short and long-term changes in brain structure. Lisofsky et al. [Lisofsky et al., 2016] showed that even brief use of oral contraceptives (the pill) can result in structural brain changes in regular cycling females (ages 16-35) using VBM. Specifically, female contraceptive users $(n=28)$ showed volume reductions in the left amygdala and anterior parahippocampal gyrus (PHG) compared to age-matched controls $(n=28)$ after 3 months of daily contraceptive use and after adjusting for age, intracranial volume (ICV), and total estrogen and progesterone levels. These regions are key hubs for emotion processing and regulation and may explain affective changes associated with contraceptive use [Montoya and Bos, 2017]. However, individuals on oral contraceptives were not on the same pill type regimen, and it is unclear how different pill types (estrogen+progestin vs. progestin-only, etc.) may have influenced these results.

Our group recently expanded on this work in the UK Biobank cohort by examining the impact of oral contraceptive use on whole-brain white matter in pre- and post-menopausal females using diffusion tensor imaging (DTI) [Nabulsi and Lawrence, 2020] - a non-invasive imaging technique that measures patterns of water diffusion throughout the brain [Basser and Pierpaoli, 1996]). The most common diffusion MRI metric is fractional anisotropy (FA), which measures the degree of diffusion restriction within an image voxel. In a trajectory analysis using fractional polynomial modeling, Nabulsi et al. [Nabulsi and Lawrence, 2020] revealed higher whole-brain FA and tensor distribution function (TDF, a more rigorous multi-tensor diffusion model that accounts for intravoxel fiber crossing) FA in contraceptive users compared to never-users ( $n=7,136$ users, $n=1,177$ non-users; ages $45-80$ years) after adjusting for age, years of education, socioeconomic status, waist-to-hip ratio and genetic ancestry. Conversely, longer duration and younger age at first contraceptive use were associated with lower FA and TDF-FA compared to never users. As higher FA is typically a good indicator of "healthy" white matter [Basser and Pierpaoli, 1996; Bennett et al., 2010], these results suggest that brief oral contraceptive use may serve a protective role for white matter microstructure, but chronic use - particularly at older ages 
- may be associated with a faster rate of white matter decline in older adulthood.

In addition to contraceptive use, hormone replacement therapy (HRT) may have neuroprotective effects when administered during perimenopause [Eberling et al., 2003; Shao et al., 2012]. Specifically, earlier studies suggested that HRT implemented early in menopause [Eberling et al., 2003; Shao et al., 2012] or over long periods may attenuate risk of AD diagnosis [Imtiaz et al., 2017] and death [Mikkola et al., 2017] in females. Indeed, neuroimaging work by Pintzka and Haberg [Pintzka and Håberg, 2015] showed that females who initiated HRT prior to menopause and remained on HRT for at least three years $(n=80)$ had greater whole hippocampal volume compared to HRT-naive females $(n=80)$ who were matched for ICV and age (ages 5166). A recent voxelwise study by Boyle et al. (2020) also revealed larger gray and white matter volume in various regions in females with a history of HRT (N=562, ages 71-94) after adjusting for data acquisition site, age, ethnicity, years of education, clinical diagnosis, history of heart disease, type 2 diabetes, and hypertension, presence of white matter lesions, BMI, physical activity, and past-year estrogen use, but the duration of HRT was not associated with imaging variables.

Other work suggests that HRT does not prevent cognitive decline [Henderson et al., 2016] and that long-term HRT may slightly increase risk for dementia [Savolainen-Peltonen et al., 2019; Wu et al., 2020]. Indeed, a randomized, double-blinded placebo controlled trial of HRT (specifically, conjugated equine estrogens) in 95 recently post-menopausal women (ages 42-56) showed decreases in whole brain volume, increased ventricle expansion, and increased white matter hyperintensity volume over 48 months of HRT compared to the placebo group. However, cognitive performance did not differ between groups [Kantarci et al., 2016a]. Finally, Nabulsi et al.'s [Nabulsi and Lawrence, 2020] earlier mentioned work on contraceptive use also examined the impact of HRT on brain white matter in the UK Biobank using both DTI and neurite orientation dispersion density imaging (NODDI). Results revealed lower whole-brain white matter fiber coherence (orientation dispersion index) in females on $\operatorname{HRT}(\mathrm{N}=3,106)$ compared to never-users 
$(\mathrm{N}=5,195)$, but less pronounced white matter changes with age [Nabulsi and Lawrence, 2020]. Further, females on an estrogen-only regimen showed greater white matter disruption with age (lower fiber dispersion, and increased free water).

The inconsistent neuroimaging markers of HRT emphasize that there is much to learn regarding the therapeutic potential of exogenous estrogen use. A major limitation of previous work is the variability in HRT chemical composition used across individuals, even within the same study. Prior work on HRT has compared studies using various hormonal combinations (e.g., estrogen, androgen, progesterone treatment alone or together), chemical formulations, doses, and delivery routes that exert different effects on the body [Comasco et al., 2014; Maki and Dumas, 2009; Moraga-Amaro et al., 2018; Yare and Woodward, 2020]. In fact, estrogen and progesterone have various molecular and binding mechanisms that modulate neurotransmitter activity, sometimes in opposing directions [Del Río et al., 2018]. This variability and lack of consensus of HRT effects on brain structure is a major gap in the literature that requires immediate attention given the growing population of older adults and the high percentage of females taking HRT worldwide [Boyle et al. 2020].

\section{Normative sex differences in human brain structure}

\section{Brain Size Metrics and Gray matter}

Neuroimaging has been particularly informative for understanding how sex differences in brain size can be explained by differences in underlying gray and white matter microstructure. At the outermost level, ICV remains an important index of maximal brain size across the lifespan, as the intracranial vault is set at approximately age 7 and is not susceptible to developmental or degenerative processes [O'Brien et al., 2006]. Sex differences in ICV have been established in both large and small-scale studies. On average, males have significantly larger ICV than females 
[Ruigrok et al., 2014], and these differences have been shown to account for some, but not all, regional sex differences in brain volumes [Jahanshad and Thompson, 2017]. The literature also shows that total brain volume (TBV) - measured on T1-weighted brain scans - is approximately $9-12 \%$ larger in males than females in childhood, adolescence, and adulthood in both large and small scale studies (for review see [Kaczkurkin et al., 2019; Lenroot and Giedd, 2010]. This aligns with very early evidence that brain weight is approximately $10 \%$ larger in males than females at autopsy [Pakkenberg and Voigt, 1964; Voigt and Pakkenberg, 1983].

Similarly, sex differences in gray matter measures are well-established across the lifespan. In young adults (age 20-34 years), VBM has revealed larger total normalized gray matter volume (total gray matter volume / total ICV) but also a faster rate of age-related decline in normalized gray matter volume in females $(n=71)$ than males $(n=71)$ [Farokhian et al., 2017]. Yang et al. [Yang et al., 2020] revealed sex differences in several different cortical gray matter features including sulcal depth and cortical thickness in young adults (aged 19-37) from the Chinese and US HCP cohorts using sample-specific surface templates based on Freesurfer segmentations from 35 Desikan-Killiany (DK) and 75 Destrieux atlas structures in each hemisphere. In the Chinese HCP, males $(n=100)$ exhibited greater cortical thickness than females $(n=100)$ in the frontal, temporal, and parietal lobes after correcting for age and ICV, whereas in the US HCP, males $(n=100)$ exhibited lower thickness than females in the caudal anterior cingulate cortex $(A C C)$, but greater thickness in the insula, lateral orbitofrontal cortex (OFC), and the isthmus of the cingulate than females $(n=100)$.

The Enhancing Neuroimaging Genetics through Meta-Analysis (ENIGMA) consortium has begun to chart neuroimaging sex differences across the lifespan to provide a benchmark for evaluating individual brain health and improve disease detection and monitoring [Dima et al., 2020; Frangou et al., 2020b; Wierenga et al., 2020a]. Recent mega-analyses of subcortical volumes and cortical thickness by the ENIGMA Lifespan Working Group has shown that while unadjusted overall cortical volume and thickness are larger in males than females, sex differences 
do not persist after covarying for ICV [Dima et al., 2020; Frangou et al., 2020a]. Trajectory analyses, however, do show significant sex differences in the rate of change in cortical thickness across the lifespan. Specifically, Frangou et al. [Frangou et al., 2020a] showed that males $(n=8,212)$, on average, had faster whole-brain cortical thinning than females $(n=8,863)$ during midlife (ages 30-59), but cortical thinning rates were comparable between males and females in early life (ages 3-29) and in older adulthood (ages 60-90). Males also had faster regional cortical thinning than females in motor, somatosensory, and visual association cortices during early life (ages 3-29), and in frontal-temporal cortical areas during midlife [Frangou et al., 2020a]. Of note, sex was the only covariate used for trajectory modeling, as the imaging data were harmonized using the ComBat package in $\mathrm{R}$, which adjusts for site and scanner-related variance.

In more recent work from the ENIGMA-Lifespan group, Wierenga et al. [Wierenga et al., 2020b] examined cortical and subcortical brain metrics in 16,683 individuals between ages 1-90 years (47\% female) using the ENIGMA FreeSurfer [Fischl, 2012] cortical and subcortical pipelines http://enigma.ini.usc.edu/protocols/imaging-protocols/ [ENIGMA, 04/2017]. Results revealed sex differences both in brain structural metrics and in between-subject variability in brain metrics after adjusting for cohort, magnetic field strength, FreeSurfer version, and age. Specifically, males had larger volumes than females in all subcortical ROls (Cohen's $d$ range $=0.41$ (left accumbens) to 0.92 (right thalamus), and these differences persisted with slightly smaller effect sizes after covarying for TBV ( $d$ range $=0.21$ (left accumbens) to 0.58 (right thalamus)). Males also had greater regional surface area than females across the entire cortex with and without adjusting for total surface area (without: $d$ range=0.42 (left ACC) to 0.97 (left superior temporal gyrus, STG); with: $d=0.21$ (left ACC) to 0.59 (left STG)). Females had greater thickness than males in 38 of the 68 Desikan-Killiany atlas-defined cortical regions, but effect sizes were comparatively small (largest effect, $d=0.12$ in the right caudal ACC), and effects in several regions changed direction (males $>$ females) or became non-significant when total thickness was included as a covariate. However, males showed significantly greater between-subject variability than females for all 
subcortical volumes and cortical surface area metrics, and for $60 \%$ of cortical thickness metrics, and these differences persisted throughout the lifespan [Wierenga et al., 2020a].

Population-based studies of middle-aged and older adults (ages 45-80 years) from the UK Biobank cohort generally align with the findings from the ENIGMA-Lifespan group. Specifically, Ritchie et al. [Ritchie et al., 2018] reported greater cortical surface area in males $(n=2,466)$ than females $(n=2,750)$ in most regions after adjusting for TBV, age, and ethnicity. Conversely, females had greater thickness than males across most of the cortex except the medial OFC and rostral ACC, which was thicker in males. Females also had larger volumes in the nucleus accumbens compared to males after adjusting for TBV, whereas males had larger volumes in the putamen, amygdala, and pallidum after adjusting for the same covariates [Ritchie et al., 2018]. Other work in the UK Biobank revealed significant age-by-sex interaction effects in subcortical volume trajectories, such that males $(n=12,665)$ exhibited faster decline than females $(n=13,775)$ in all volumes across the full age range (44-81 years) after adjusting for ICV, education, and BMI [Ching et al., 2020]. Interestingly, sex differences in volume loss were significantly attenuated after age 60 , suggesting that the greatest sex effects on subcortical volumetry may occur during middle age.

\section{Hippocampal Volume}

The hippocampus is a complex subcortical brain structure that serves as an essential integratory hub for memory (formation, storage, retrieval), spatial navigation, and emotional processing. As sex differences are reported in these functions, numerous imaging studies have investigated hippocampal volume in clinical and non-clinical populations [Yagi and Galea, 2019]. Consistent with population-based studies from ENIGMA and the UK Biobank, a meta-analysis of hippocampal volumes in healthy participants from 76 studies $(N=4,418$, ages $0-79$ years, $45.3 \%$ female) showed that raw hippocampal volumes were larger in males than females by $\sim 6-7 \%$, but statistical adjustments for ICV or TBV nullify these sex differences [Tan et al., 2016]. Similarly, 
nomograms (percentile charts) of whole hippocampal volume (computed with FSL-FIRST) in 19,793 individuals from the UK Biobank (ages 45-80 years, 52.9\% female) showed similar hippocampal volume measurements by sex after adjusting for age, scan date, and an automated metric of head size derived from the nomogram pipeline (https://Inobis.github.io/HippoFit Tool/index.html) [Nobis et al., 2019]. However, trajectory analyses revealed significant sex differences in the rate and temporal change of hippocampal volume loss with age, with accelerated loss in males around age 50 and accelerated loss in females between ages 60-65. The rate of hippocampal volume loss relative to total gray matter volume also differed in males and females, with peak inflection points around ages 63 and 67, respectively [Nobis et al., 2019].

Finally, while sex differences in total hippocampal volume do not persist after correcting for head size, prior work suggests that subregions of the hippocampus are sexually dimorphic. In a recent lifespan study of hippocampal subfield volumes manually segmented from $4.7 \mathrm{~T}$ scans $\left(\mathrm{N}=129\right.$, ages 18-85, $\left.\mathrm{M}_{\mathrm{age}}=47.6 \pm 18.9\right)$, Malykhin et al. [Malykhin et al., 2017] found larger subfield volumes in the hippocampal head (dentate gyrus, DG), body (CA1-3, subiculum, DG), tail (all subfields), and dentate gyrus in females $(n=70)$ than males $(n=59)$ after normalizing hippocampal volumes by ICV (raw hippocampal volume / ICV of same subject $x$ sample averaged ICV) and removing the effects of age. Sex-by-age interactions were significant in the subiculum of the hippocampal tail, with a marginally significant non-linear effect of age on subiculum volumes in females, but no significant effect in males [Malykhin et al., 2017].

Interestingly, a larger study of young adults (ages 21-36) from the Queensland Twin Imaging study (QTIM, 4 T scanner) and Human Connectome Project (HCP, 3 T scanner) revealed larger subfield volumes (segmented with the FreeSurfer-v.6.0 hippocampal subfield pipeline) in males $(n=692)$ than females $(n=995)$ in the fimbria, parasubiculum, fissure, and presubiculum after statistically adjusting for whole hippocampal volume [van Eijk et al., 2020]. Importantly, sex differences persisted across four different statistical methods to control group differences in whole 
hippocampal volume: (1) allometric scaling- regresses out the effect of whole hippocampal volume (or comparable metric) after identifying the scaled relationship between whole hippocampal volume and each subfield via log-log regression, (2) covariate- models the effect of whole hippocamal volume as a covariate predictor in regression or ANCOVA, (3) residualsregresses out the effect of whole hippocampal volume on the subfield $\mathrm{ROI}$ and uses the residuals as the dependent variable, and (4) matched-where groups are matched by whole hippocampal volume. When subfields were adjusted for brain segmentation volume (a FreeSurfer defined metric of total brain size that includes gray matter, white matter, and CSF) rather than whole hippocampal volume, males again had larger volumes than females in the hippocampal fissure, presubiculum, and parasubiculum using all four correction methods, albeit at smaller effect sizes for subicular subregions. Males also had larger volumes than females in the fimbria and subiculum using covariate, residual, and matching methods, but not when using allometric scaling. Sex differences were not detected in the CA2/3, CA4, hippocampal-amygdala transition area (HATA), or DG using any normalization technique or covarying for whole hippocampal volume or brain size [van Eijk et al., 2020]. While these findings differ considerably from those reported by Malykhin et al. [Malykhin et al., 2017], they may be explained by key methodological distinctions including (but not limited to) considerable differences in sample size and composition (lifespan approach (mean age $\sim$ mid 40's) vs. young adults (mean age $\sim$ mid 20's)), use of manual vs. automated hippocampal subfield parcellation, and use of different normalization techniques and covariates.

\section{Laterality}

Brain laterality is an important metric of brain organization and contributes to many cognitive processes. Thus, understanding sex differences in brain asymmetries can inform mechanisms of sex differences in cognitive function (visuospatial processing, spatial navigation) and risk factors for various brain diseases (e.g., Alzheimer's disease, schizophrenia) [Kong et al., 
2018]. In the general population, our ENIGMA-Laterality Working Group recently revealed greater right-ward asymmetry in the putamen and greater left-ward asymmetry in the globus pallidus in males compared to females in a volumetric meta-analysis of subcortical brain structures in 15,847 participants (median age across studies=33.9 years, 53\% female, [Guadalupe et al., 2017]. A subsequent meta-analysis of cortical data by the same group revealed more leftward asymmetry in cortical thickness of the PHG and entorhinal cortex (ERC), and more rightward asymmetry of global surface area in males versus females in 17,141 individuals ages 3-90 years [Kong et al., 2018]. Regional analyses showed the most pronounced rightward asymmetry in surface area of frontal, temporal, parietal, and anterior cingulate cortices. Results were replicated in two independent samples, including the young adult HCP cohort [Kong et al., 2018].

\section{White Matter}

Normative sex differences in white matter microstructure have been shown using DTI metrics. Our ENIGMA-DTI Working Group ( $\mathrm{N}=481,60 \%$ female, ages 22-36 years) showed that FA is a heritable metric, with age, sex, age-by-sex, age ${ }^{2}$, and age ${ }^{2}$-by-sex explaining $10 \%$ of the total variance in whole-brain average FA, and genetic factors explaining $78 \%$ of the remaining variance in FA [Kochunov et al., 2015]. Sex was the only significant covariate predictor of wholebrain FA, with $\sim 2 \%$ higher whole-brain average FA in females than males. Regionally, sex predicted higher FA in females than males for most white matter tracts, with the strongest sex effects in the internal capsule and fornix (19.1\% and $14 \%$ variance explained, respectively), and the weakest effects in the superior fronto-occipital fasciculus and inferior fronto-occipital fasciculus (IFOF; $1.3 \%$ and $1.6 \%$, respectively). Whole-brain FA was highly heritable in both females and males when examined separately, with genetic factors explaining $85.7 \%$ and $91.9 \%$ of total FA variance (respectively). A weak proportion of FA variance was attributed to linear and non-linear age effects in both males $(1.5 \%)$ and females $(0.15 \%)$, which is consistent with the age range of the cohort [Kochunov et al., 2015]. 
Later work in a slightly younger cohort $(\mathrm{N}=667$, ages $18-30,62 \%$ female) of 415 families revealed a slightly different relationship between age and sex on FA measurements along the length of specific fiber tracts using high angular resolution diffusion imaging [Dennis et al., 2017]. Results revealed significant age-by-sex interactions in right frontal callosal fibers and the right IFOF, with greater positive associations between FA and age in females than males after adjusting for family relatedness (random effect), age, and $\operatorname{age}^{2}$ (fixed effects). Although effect sizes were not reported, marginally higher FA in the IFOF of females than males is consistent with DTI findings in middle age and older adults in the UK Biobank cohort (ages 44-77 years) [Ritchie et al., 2018]. Here, females $(n=2,750)$ had higher FA than males $(n=2,466)$ in the left inferior longitudinal fasciculus (ILF; $d=0.14$ ) and posterior thalamic radiation (PTR; $d=0.12)$ after adjusting for TBV, age, age-by-sex interactions, and ethnicity. By contrast, males had significantly higher FA than females in the right arcuate fasciculus (ARC; $d=0.26$ ), bilateral corticospinal tract (CST) (right $d=0.22$, left $d=0.15$ ), and bilateral superior thalamic radiation (STR) (right $d=0.16$, left $d=0.15)$. Interestingly, analyses that did not adjust for group differences in TBV revealed stronger sex differences in FA (male>female) in the ARC, CST, and STR ( $d$ range=0.26-0.56) and weaker sex differences (female>male) in the ILF and PTR $(d ' s=0.10)$ and sex differences in the IFOF became non-significant compared to analyses that covaried for TBV. Interestingly, advanced diffusion metrics from NODDI revealed greater tract complexity (higher orientation dispersion) in females than males in all white matter tracts after adjusting for all covariates (average $d=0.30$ ), but the functional significance of this finding is currently unknown [Ritchie et al., 2018].

\section{Sex differences in adult psychiatric illness}

Many psychiatric conditions show sex differences in prevalence and incidence rates (Figure 1), age of onset, clinical presentation, or treatment efficacy, yet information on sex 
differences in brain imaging signatures is surprisingly limited. Below we discuss the extant literature on sex differences in neuroimaging, as well as clinical features and expression patterns for adult onset psychiatric disorders. As noted in the Introduction, we focus our review on common psychiatric conditions that have been linked to increased risk for neurodegenerative conditions and dementia, as these may yield early neuroimaging cues that can be used for risk profiling, disease monitoring, and ideally the development of new treatments and interventions.

\section{Post-traumatic Stress Disorder}

Post-traumatic stress disorder (PTSD) is a complex condition that develops after exposure to a serious and often life-threatening event [Nisar et al., 2020]. Core symptoms include avoidance, hyperarousal, re-experiencing, and negative alterations in mood and cognition. A combination of these must persist for at least one month to meet criteria for PTSD [National Institute of Mental Health, 2019]. The lifetime prevalence of PTSD is significantly higher for females $(2.6 \%)$ than males $(1.0 \%)$ cross-nationally $(\mathrm{N}=71,083)$ [Koenen et al., 2017], with around twice the prevalence for females (lifetime $=6.1 \%, 12$-month $=6.1 \%$ ) than males $($ lifetime $=4.1 \%, 12$ month=3.2\%) in the US [Goldstein et al., 2016], despite experiencing similar numbers of traumatic events [Lehavot et al., 2018]. In contrast to other psychiatric disorders, there are inherent sex biases related to trauma exposure type and the pathophysiology of PTSD. For example, females tend to be overrepresented in civilian cases of PTSD as a result of intimate partner violence and/or rape [Laskey et al., 2019], whereas males are significantly overrepresented in studies of combatexposed PTSD [Lehavot et al., 2018]. Still, a US-based epidemiology study of PTSD ( $N=36,101$, $56.5 \%$ female) revealed significantly higher prevalence in 12-month and lifetime PTSD in female versus male veterans (12-month $11.7 \%$ vs. $6.7 \%$; lifetime: $13.4 \%$ vs. $7.7 \%$ ) and civilians (12month: $6 \%$ vs. $2.6 \%$; lifetime: $8 \%$ vs. $3.4 \%$ ) [Lehavot et al., 2018]. 
Sex differences in clinical patterns have also been reported, with females demonstrating greater acute PTSD symptoms $(d=0.24)$, peritraumatic dissociation $(d=0.21)$, and perceived life threat $(d=0.32)$ than males in a hospital setting, as well as greater PTSD symptoms 6 weeks $(d=0.52)$ and 6 months $(d=0.66)$ after trauma exposure [Irish et al., 2011]. Females also show greater acquisition of conditioned fear and greater fear response to a conditioned stimulus compared to males [Inslicht et al., 2013]. The severity of fear response and corresponding symptoms also varies by trauma exposure type in females, but not in males [Lancaster et al., 2014].

The most common structural neuroimaging signatures of PTSD include volumetric abnormalities in the hippocampus, amygdala, and prefrontal cortex (PFC) [Akiki et al., 2017; Bae et al., 2019; Dennis et al., 2019], regions that collectively describe the "fear circuit." Indeed, a meta-analysis of subcortical brain volumes by our ENIGMA-PGC PTSD Working Group (a partnership with the Psychiatric Genetics Consortium, PGC) recently revealed significantly smaller volumes in the hippocampus, and a trend for smaller volumes in the amygdala, in 794 PTSD individuals compared to 1,074 controls (mostly trauma-exposed) after adjusting for age and ICV [Logue et al., 2018]. A sex-by-diagnosis interaction effect on hippocampal volume was not significant, but sex-specific analyses revealed smaller hippocampal volumes in female PTSD patients $(n=308)$ versus female controls $(n=428 ; p<0.001)$, with stronger effect sizes in females $(d=-0.31)$ than the full sample $(d=-0.17)$. Hippocampal volumes did not differ significantly between cases $(n=472)$ and controls $(n=629)$ in males $(p=0.23)$, but Cohen's $d$ confidence intervals overlapped in males and females suggesting limited power to detect a PTSD effect in males, despite being a larger subsample than females [Logue et al., 2018].

The ENIGMA-PGC PTSD Working Group recently published a mega-analysis of cortical volumes, revealing smaller volumes in 1,379 PTSD patients compared to 2,192 controls (aged 685 years) across most of the cortex (particularly in frontal-temporal areas) after adjusting for age, sex, ICV and a random intercept to model cohort and scanner differences across sites [Wang et 
al., 2020a]. Although sex had a significant main effect on most cortical regions, sex-by-diagnosis interactions were not significant in any region. Similarly, a meta-analysis by our group of PTSD effects on white matter tracts revealed significantly lower FA in the tapetum (a tract connecting the left and right hippocampi), but did not detect significant sex effects in any tract [Dennis et al., 2019].

\section{Anxiety Disorders}

In the current version of the Diagnostic Statistical Manual (DSM-5), nine distinct conditions are recognized as anxiety disorders: (1) generalized anxiety disorder (GAD), (2) social anxiety disorder, (3) separation anxiety disorder, (4) panic disorder, (5) specific phobia, (6) agoraphobia, (7) selective mutism, (8) anxiety disorders due to substance use, and (9) anxiety disorders due to another medical condition. Anxiety disorders are the most common mental health conditions worldwide [Ritchie and Roser, 04/2018], affecting over 3 million individuals in 2019 [Institute for Health Metrics and Evaluation (IHME), 2019]. The nosology of anxiety disorders has evolved over the past decade, particularly with the transition from version 4 to version 5 of the DSM in 2013. Importantly, previously categorized anxiety disorders such as PTSD and obsessive compulsive disorders (OCD) are now recognized as independent diagnostic conditions in the DSM-5. This has complicated the global picture of sex differences in psychiatric disease prevalences, as other diagnostic systems (e.g., International Classification of Diseases-10) do not distinguish OCD and PTSD from other anxiety disorders. Nevertheless, most anxiety disorders are more common in females than males [Bekker and van Mens-Verhulst, 2007; Jalnapurkar et al., 2018; McLean et al., 2011].

Here we focus on GAD and panic disorder $(\mathrm{PaD})$, two of the most common anxiety disorders in adults. Social anxiety disorders and specific phobias affect a greater proportion of the population than GAD and PaD [Kessler et al., 2005; McLean et al., 2011] but are primarily 
linked to childhood/developmental origins [Lijster et al., 2017]. As such, we focus on anxiety disorders with adult onset to maintain a clear and concise narrative.

\section{Generalized Anxiety Disorder}

GAD is characterized by excessive, uncontrollable worry that interferes with activities of daily living. Symptoms of GAD often include restlessness, insomnia, fatigue, irritability, and difficulty concentrating [Terlizzi and Villarroel, 2020]. In a survey of 30,000 adults from the United States, nearly $15 \%$ reported some form of GAD symptoms in the two weeks preceding the survey; nearly $10 \%$ reported mild GAD symptoms and $3 \%$ reported severe symptoms. Approximately $6 \%$ of the total sample reported symptoms severe enough to meet clinical criteria for probable GAD. When stratified by sex, up to $19 \%$ of females reported GAD symptoms of any severity, compared to only $12 \%$ of males [Terlizzi and Villarroel, 2020]. Globally, the lifetime prevalence of GAD is $3.7 \%(1.8 \%$ past year), with a disproportionate impact on females $(\mathrm{OR}=1.8)$ and in higher-income countries such as Australia (8\%), New Zealand (7.9\%), and the United States (7.8\%) [Ruscio et al., 2017]. Sex differences in GAD prevalence in the United States are similar to global estimates, with a nearly 2-fold greater prevalence in females than males (12-month, $O R=1.74$; lifetime, OR=1.83) [McLean et al., 2011]. Symptoms of GAD also differ by sex, with females reporting greater somatic issues (muscle tension, fatigue), neuroticism, and negative affect than males. Females also tend to have earlier symptom onset and lower remission rates than males [Yonkers et al., 2003]. GAD also has a high comorbidity profile that differs significantly by sex. Specifically, females with GAD are more likely than males to have comorbid depression and other anxiety disorders, whereas males with GAD are more likely than females to have comorbid alcohol, nicotine and other drug use disorders as well as antisocial personality disorder [Jalnapurkar et al., 2018].

Neuroimaging studies of GAD have shown abnormalities in both gray matter and white matter microstructure using structural and diffusion MRI. A brief review by Maron and Nutt [Maron 
and Nutt, 2017] noted that larger volumes in subcortical brain structures of GAD patients versus controls, particularly in the amygdala and dorsomedial PFC, were among the most common structural MRI signatures of GAD. However, a more recent systematic review of 26 structural imaging studies [Madonna et al., 2019] reported inconsistent effects of GAD in gray and white matter of these regions, and in the dorsolateral and ventrolateral PFC, ACC, and posterior parietal regions (larger and smaller volumes reported in GAD patients vs. controls). These paradoxical findings are likely due to heterogeneous research designs, imaging protocols, sample composition (inclusion vs. exclusion of comorbid psychiatric disorders), and remarkably small sample sizes across studies (average number of GAD participants per study=10-25). Interestingly, however, DTI studies consistently reported lower FA in the uncinate fasciculus of GAD patients versus controls [Madonna et al., 2019].

We were surprised to find that almost no studies have examined the role of sex on structural brain signatures in GAD using neuroimaging, and to our knowledge this work has only been conducted in adolescents [Liao et al., 2014]. The lack of dedicated neuroimaging studies in adult samples of GAD is a major gap in the literature, particularly given the significant role of sex in disease prevalence and clinical symptoms. In their 2017 review, Maron and Nutt [Maron and Nutt, 2017] noted that larger volumes in the amygdala and dorsomedial PFC in GAD were commonly observed in all-female or predominantly-female samples, but the role of sex was not explicitly tested. Thus, testing and comparing the directionality of GAD effects in the amygdala and PFC between males and females may be an ideal starting point for future neuroimaging investigations of GAD.

\section{Panic Disorder}

Panic disorder $(\mathrm{PaD})$ is a debilitating anxiety disorder characterized by abrupt and unpredictable episodes of heightened physiological arousal (i.e., panic attacks) that cause significant mental distress and physical discomfort. $\mathrm{PaD}$ affects $2.7 \%$ of the global population at 
some point during life, with median onset around age 32 [de Jonge et al., 2016]. Similar to GAD, $\mathrm{PaD}$ is disproportionately represented in high-income countries, with the highest 12-month and lifetime prevalence in the United States. Cross-nationally, both lifetime and 30-day prevalence of $\mathrm{PaD}$ are significantly more prevalent in females (lifetime, $\mathrm{OR}=1.8,30$-day, $\mathrm{OR}=2.0$ ) than males (lifetime, $\mathrm{OR}=1.0,30$-day, $\mathrm{OR}=1.0$ ). Approximately $70-80 \%$ of individuals with $\mathrm{PaD}$ meet criteria for at least one comorbid mental health condition, most commonly another anxiety or mood disorder [de Jonge et al., 2016].

Clinical symptom expression of PaD also differs between males and females. Results of the United States National Comorbidity Study $(\mathrm{N}=8,089$, ages $15-54)$ showed that females with $\operatorname{PaD}(n=194)$ experienced greater frequency of panic-related symptoms than males with $\mathrm{PaD}$ $(n=80)$, including shortness of breath, nausea, and feeling smothered. Conversely, males with $\mathrm{PaD}$ reported greater frequency of stomach pain and sweating compared to females with $\mathrm{PaD}$ [Sheikh et al., 2002]. A more recent study of $\mathrm{PaD}$ patients from South Korea indicated that females with $\mathrm{PaD}(\mathrm{n}=291)$ reported a greater number of stressful life events compared to males with $\mathrm{PaD}$ $(n=254)$, including physical injury, pregnancy-related issues, and separation from parent, friend, or romantic partner. A series of self-report questionnaires also showed that males with PaD were more likely to endorse a confrontational coping style, and were more likely to seek social support compared to females with $\mathrm{PaD}$. Here, males and females reported comparable levels of depression and panic-related symptoms, but females reported significantly greater symptoms of agoraphobia than males. Finally, females reported significantly lower physical functioning than males [Kim et al., 2017].

Neuroimaging markers of $\mathrm{PaD}$ include structural abnormalities in the amygdala, nucleus accumbens, thalamus, striatum, hippocampus, cerebellum, insula, ACC, midcingulate, inferior frontal gyrus (IFG), superior temporal gyrus (STG), and somatosensory cortex using VBM [Del Casale et al., 2013; Wang et al., 2020b]. The direction of effects in these regions has been somewhat inconsistent across studies, with most studies reporting smaller volumes in these 
regions in $\mathrm{PaD}$ individuals compared to controls. However, a handful of studies reported larger volumes in patients than controls, particularly in brainstem nuclei [Del-Ben and Graeff, 2009; Del Casale et al., 2013; Protopopescu et al., 2006; Sobanski and Wagner, 2017; Uchida et al., 2008; Wang et al., 2020b]. Larger brainstem volumes in PaD individuals versus controls may explain autonomic features of PaD, but much more work is needed in this area to understand this effect.

Few studies have examined neuroimaging sex differences in $\mathrm{PaD}$, and all have been conducted in very small samples. An earlier VBM study of $24 \mathrm{PaD}$ patients ( 9 male, 15 female) and 24 matched controls reported [Asami et al., 2009] smaller volumes in several brain regions (amygdala, ACC, STG, insula, cerebellar vermis, and regions of the fronto-occipital cortex) of the PaD group after adjusting for age, ICV, and socioeconomic status. Of these regions, males with $\mathrm{PaD}$ showed significantly lower volumes than females with $\mathrm{PaD}$ in the bilateral insula, right amygdala, and left occipitotemporal gyrus, whereas females with PaD only had smaller volumes than males in the right STG. However, sex-specific analyses revealed female-specific effects (PaD females < Control females) in the bilateral dorsolateral PFC (DLPFC), ventrolateral PFC, thalamus, parietal cortex, and right cerebellar vermis [Asami et al., 2009].

\section{Obsessive Compulsive Disorder}

Obsessive compulsive disorder (OCD) is a common psychiatric disorder with a complex phenomenology. Previously characterized as an anxiety disorder, OCD is now recognized by the DSM-5 as an independent class of obsessive compulsive and related disorders (OCRD) that include OCD, body dysmorphia, hoarding, trichotillomania, excoriation disorder (skin picking), and OCD secondary to medication use or another medical condition [Marras et al., 2016]. Hallmark features of OCD include persistent and intrusive thoughts, urges, or images (i.e., obsessions), and repetitive and/or rigid behaviors that occur in response to an obsession [NIMH, 2019]. OCD is also highly comorbid with other psychiatric conditions, with lifetime comorbidity estimates as high as $92 \%$ in population-based samples [Brakoulias et al., 2017; de Mathis et al., 2013; Ruscio 
et al., 2010]. The high comorbidity rate and evolving nosology of OCD has complicated efforts to characterize the role of demographic factors in OCD, but most studies show higher lifetime prevalence of adult $O C D$ in females $(O R=1.4-3.0)$ than males $(O R=0.9-1.0)$ [Fawcett et al., 2020; Kessler et al., 2012]. Sex differences in past year prevalence of OCD, however, has been inconsistent across studies [Adam et al., 2012; Castle et al., 1995; Fawcett et al., 2020; Mathes et al., 2019; Ruscio et al., 2017]. These inconsistencies may be due to the proportion of adolescents surveyed, as OCD is purportedly more common in males than females, whereas the opposite is true in adults [Mathes et al., 2019]. In adults over age 65, recent work suggests greater OCD prevalence in males than females [Cath et al., 2017].

Prior work has identified notable sex differences in the expression of OCD symptoms. Females are more likely to have contamination obsessions and preoccupation with things that may harm others compared to males. Thus, females tend to express more cleaning and checking compulsions. Obsessions and compulsions in males are more frequently related to intrusive sexual and religious dimensions, as well as preoccupations with symmetry and order [Torresan et al., 2013]. Sex differences in comorbidity profiles also have been reported, with females having significantly higher comorbidity rates than males of any mental disorder [Benatti et al., 2020], with specifically higher comorbidity rates of mood and eating disorders than males. By contrast, males with OCD are more likely to have comorbid alcohol use disorders, psychotic disorders and developmental disorders than females [Rintala et al., 2017; Torresan et al., 2013].

Neuroimaging hallmarks of OCD include structural alterations in gray matter and white matter of fronto-striatal, thalamic, and temporolimbic and temporal-parietal networks, as well as the corpus callosum [Boedhoe et al., 2017; Boedhoe et al., 2018; Piras et al., 2015; Piras et al., 2019]. In large-scale studies, including those from our group, the most consistent structural abnormalities in OCD patients include cortical thinning of the DLPFC, transverse temporal, and inferior parietal cortices, smaller hippocampal volumes, and larger pallidum volumes relative to controls [Boedhoe et al., 2017; Boedhoe et al., 2018; Fouche et al., 2017; Piras et al., 2019]. 
There is currently little to no evidence of sex differences in neuroimaging indices, but most studies have modeled sex as a covariate rather than predictor variable of interest or have included matched samples of males and females [Hazari et al., 2019]. In one small study [Hawco et al., 2017] comparing whole-brain FA metrics between male $(n=17)$ and female $(n=21)$ OCD patients, males with OCD had higher FA than females, consistent with normative sex differences in brain white matter. Sex-by-diagnosis interactions were tested in meta- and mega- analyses of subcortical, cortical, and DTI metrics from the ENIGMA consortium [Boedhoe et al., 2017; Boedhoe et al., 2018; Piras et al., 2019], but these were not significant in any region. Of note, effect sizes of OCD on brain structure are small in general (average Cohen's $d<0.2$ ) [Boedhoe et al., 2017; Boedhoe et al., 2018; Piras et al., 2019], which may be due to the high comorbidity rate of OCD. As sex differences in OCD appear to be stronger in relation to symptom dimensions and comorbidity profiles, future neuroimaging studies should examine sex-specific neuroimaging patterns in relation to these clinical features.

\section{Major Depressive Disorder}

Approximately $4.4 \%$ of the global population has been diagnosed with major depressive disorder (MDD) [World Health Organization., 2017], with a 2-fold greater lifetime risk of MDD in females than males [Rubinow and Schmidt, 2019]. Sex differences are also evident in patterns of symptom expression. A recent meta-analysis by Cavanagh et al. [Cavanagh et al., 2017] showed that males with MDD report a higher frequency of risk taking, alcohol/substance misuse, anger, low self-esteem, and cognitive difficulties compared to women. Conversely, females with MDD are 3 times more likely to exhibit atypical depression symptoms such as fatigue, increased appetite, and sleep disturbances compared to men, who more frequently demonstrate typical (melancholic) depression symptoms. Females also have a 2- to 3-fold greater risk for dysthymia and seasonal affective disorder [Rubinow and Schmidt, 2019]. Sex differences in functional 
impairments are also reported, with males showing greater work impairment and females showing greater social impairment.

Neuroimaging hallmarks of MDD include smaller volumes in the hippocampus, amygdala, ACC, DLPFC, medial PFC and OFC, and larger volumes in the ventricles [Dunlop et al., 2019; Lai, 2019; Lemogne et al., 2012]. The ENIGMA-MDD Working Group confirmed these hallmarks in the largest subcortical, cortical, and white matter neuroimaging studies of MDD to date $(\mathrm{N}>8,000)$, yet none of these studies detected sex by diagnosis interaction effects on brain structure [Schmaal et al., 2016; Schmaal et al., 2017; van Velzen et al., 2019]. However, separate work by Frodl et al. [Frodl et al., 2017] and Tozzi et al. [Tozzi et al., 2019] revealed sex by diagnosis interaction effects on subcortical and cortical brain structure as a function of childhood trauma exposure. In a meta-analysis of 958 MDD patients $\left(M_{\text {age }}=42.4 \pm 14.3\right.$ years, $64 \%$ female $)$ and 2,078 controls ( $M_{\text {age }}=46.3 \pm 15.2$ years, $48 \%$ female), greater severity of childhood trauma corresponded to smaller bilateral caudate volumes in females in patients and controls, but this effect was not significant in males [Frodl et al., 2017]. In a subsequent meta-analysis of cortical morphometry in 1,284 MDD patients ( $\mathrm{M}_{\text {age }}=40.9 \pm 14.6$ years, $63.3 \%$ female) and 2,588 controls $\left(M_{\text {age }}=43.3 \pm 15.9\right.$ years, $50.3 \%$ female $)$, childhood trauma severity was positively associated with cortical thickness in the rostral ACC of males in both patient and control groups, but this effect was not significant in females [Tozzi et al., 2019].

Inconsistent and heterogeneous medication use is another complicating factor for interpreting neuroimaging results in MDD samples, as medication use is often inconsistently measured across studies. An earlier study of 29 medication-naive MDD patients (55\% female; $M_{\text {age }}=29.5 \pm 6.8$ years $)$ and 33 controls $\left(51.5 \%\right.$ female; $M_{\text {age }}=29.9 \pm 8.3$ years $)$ revealed significant sex by diagnosis interactions in the PFC, amygdala, hippocampus, and caudate [Kong et al., 2013]. Compared to controls, females with MDD had lower gray matter density in the amygdala and hippocampus, whereas males with MDD had lower gray matter density in the striatum compared to controls. Recent work from the International Study to Predict Optimized Treatment 
in Depression (i-SPOT) [Saveanu et al., 2015] used 'fixel-based' analysis (FBA) to identify microstructural white matter predictors of treatment remission after 8 weeks of antidepressant treatment in 221 medication-naive MDD patients and 67 controls, aged 18-65 years [Lyon et al., 2019]. The FBA technique measures individual fiber bundle elements (with different orientations) within an image voxel (i.e., fixel) to measure the degree and density of crossing fibers in white matter tracts [Raffelt et al., 2015; Raffelt et al., 2017], which describe more than $90 \%$ of white matter voxels [Jeurissen et al., 2013]. FBA produces three key metrics based on the fiber orientation distribution: fiber density ${ }^{1}$, fiber cross-section $(\mathrm{FC})^{2}$, and fiber density cross-section $(\mathrm{FDC})^{3}$; in which lower values of all three metrics represent decreased white matter microstructure. Sex-stratified analyses by Lyon et al. [Lyon et al., 2019] showed that females with MDD ( $n=115)$ had $\sim 10 \%$ lower FDC (the product of fiber density and FC) in the genu of the corpus callosum compared to healthy controls $(n=34)$, whereas males with MDD $(n=106)$ showed $\sim 11 \%$ lower FDC in the right anterior limb of the internal capsule (ALIC) compared to controls $(n=33)$ independent of treatment outcome. Additionally, compared to female controls, females with MDD had on average $5-8 \%$ lower FC in clusters containing the corpus callosum, right ALIC, tapetum, and ILF; males with MDD exhibited $\sim 7 \%$ lower fiber cross-section in the right ALIC only. Across both sexes, lower fiber cross-section in the tapetum predicted decreased likelihood for remission after antidepressant treatment [Lyon et al., 2019]. As the tapetum is the major connector between the left and right hippocampus, these findings align with existing studies by our group and others implicating the hippocampus as a common site for brain disruption in MDD.

Finally, advanced age may also affect sex differences in clinical and neuroimaging phenotypes of MDD. In a recent analysis of 610 community-dwelling older adults (ages 67-74, $52.5 \%$ female), sex-by-lifetime MDD interactions were significant in the amygdala, caudate, and

\footnotetext{
${ }_{1}^{1}$ Fiber density (FD) depicts the intra-axonal restricted compartment of a fiber pathway.

${ }^{2}$ Fiber cross-section (FC) is the approximate fiber bundle area that is perpendicular to fiber length.

${ }^{3}$ Fiber density cross-section (FDC) is a summary measure, or the product, of FD and FC [Raffelt et al., 2017].
} 
rostral ACC [Ancelin et al., 2019]. Lifetime MDD was associated with a $4 \%$ and $7 \%$ reduction in the amygdala and caudate in males respectively, with no significant effect in females. By contrast, lifetime MDD in females was associated with $6 \%$ larger volume in the rostral ACC compared to healthy controls, with no significant effect in males. Interestingly, the proportion of females with both current and lifetime MDD was significantly higher than in males, but antidepressant use and prevalence of hypertension were significantly higher and lower (respectively) in females than in males [Ancelin et al., 2019].

\section{Bipolar Disorder}

Bipolar Disorder (BD) is a manic-depressive mental health condition characterized by severe mood shifts that interfere with daily life [The National Institute of Mental Health, 2020]. The age-standardized 12-month prevalence rate of $\mathrm{BD}$ is around $0.7 \%$ of the world population - a rate that has remained stable since 1990 [Ferrari et al., 2016]. There are two primary subtypes of BD; BD-I is "traditional" BD, which involves manic and depressive periods that generally last at least 7 days and 2 weeks, respectively. BD-Il also involves mood fluctuations but with less severe manic episodes (hypomanic). The prevalence of BD-I is generally consistent between males and females [Grant et al., 2009; Szádóczky et al., 1998], but subtle differences have been reported in patients from Ethiopia ( $N=68,378$; lifetime prevalence in males $=0.6 \%$, females $=0.3 \%$ ) [Negash et al., 2005] and Hungary ( $N=2,953$; lifetime prevalence in males $=1.3 \%$ males, females $=1.6 \%$ ) [Szádóczky et al., 1998]. Several studies show higher prevalence of BD-Il in females than males, again with some inconsistencies [Szádóczky et al., 1998]. The global prevalence estimate of BD is approximately $0.6 \%$ (both types), which is generally consistent with the US [James et al., 2018].

There are also sex differences in symptom patterns and treatment response. Females show increased frequency of hypomania and more rapid cycling between manic and depressive states. Atypical depression symptoms are more common in BD females, and there is a higher risk 
for manic-depressive cycles during menopause and around childbirth, which has been attributed to intense hormonal fluctuations [López-Zurbano and González-Pinto, 2019; Sit, 2004]. Females are more frequently treated for BD with typical psychotropic medication (antidepressants, benzodiazepines) and cognitive behavioral therapy, whereas males are more commonly treated with lithium [López-Zurbano and González-Pinto, 2019].

Small-scale neuroimaging studies have reported interactions between sex and BD in the ventricles, caudate, hippocampus, amygdala, nucleus accumbens, OFC, PFC, occipitotemporal cortex, anterior pituitary, cerebellar vermis, and temporal lobe asymmetry (for review see [Jogia et al., 2012]), but across studies there is no clear direction of male-female differences among BD patients, likely due to small sample sizes (n's<100). In larger samples, however, a meta-analysis of subcortical brain structures in 1,710 BD patients (59.1\% female) and 2,594 controls (55.4\% female) from the ENIGMA-BD Working Group [Hibar et al., 2016] revealed larger thalamus volumes in females (vs. males) with BD. The ENIGMA-BD cortical analysis [Hibar et al., 2018] did not reveal significant sex differences in the effect of BD on cortical thickness or surface area in BD patients over age 25, but significant sex-by-diagnosis interactions were observed in patients under age $25\left(n=411, M_{a g e}=21.1 \pm 3.1\right)$. Specifically, young females with BD had less cortical thinning than would be expected by sex and diagnosis alone in the right pars triangularis $(\mathrm{d}=0.26)$, right superior frontal gyrus $(d=0.19)$, left insula $(d=0.20)$, and left temporal pole $(d=0.20)$ than males. The authors noted that these sex differences may reflect a normative sexual dimporphism in cortical development where cortical thickness is larger in females than males, though these interactions were not observed at older ages. Sex differences in cortical surface area were not detected. In a separate recent large-scale meta-analysis of adult BD ( $N=50$ neuroimaging studies; 1,843 BD patients, 2,289 controls), the proportion of females with BD (54.7\%), and specifically BD-I (55.1\%), correlated negatively with volume in the insula [Wang et al., 2019].

These results and the general variability of sex-by-diagnosis interactions in the BD literature call for longitudinal studies to better understand the trajectory of brain alterations in 
males and females with BD; such large-scale longitudinal studies are now underway in the ENIGMA Bipolar Working Group. Additionally, many studies do not specify BD subtypes, so it is unclear whether more pronounced sex differences in brain structure would emerge when accounting for subtype variance, particularly as BD-II has stronger sex differences in disease prevalence than BD-I.

\section{Schizophrenia}

Schizophrenia (SCZ) is a serious mental health disorder, broadly characterized by disorganized thoughts, beliefs, and behaviors [National Institute of Mental Health, 2016]. Although commonly described as a developmental condition, symptoms do not typically present until the third decade, with an incidence rate approximately $40 \%$ higher in males than females [McGrath et al., 2004; Seeman, 2013], though these numbers have been debated. SCZ also may be subdivided into early-onset and late-onset cases, with even greater sex disparities in late-onset cases. Symptom onset is generally earlier in males than females for young adult cases [Li et al., 2016], but females make up around $66-87 \%$ of late-onset cases after age 40 , with more positive symptoms than men, particularly sensory hallucinations and persecutory delusions [Lindamer et al., 1999]. Across all ages, males with SCZ tend to be less responsive to antipsychotic medications and have more frequent hospitalizations than females. The prognosis of SCZ is also generally better in females than in males, with higher rates of recovery and remission as well as better preserved interpersonal relationships, employment and marriage retention [Grossman et al., 2006; Grossman et al., 2008].

The most prominent neuroimaging markers of SCZ are enlarged ventricles and volume deficits in the hippocampus, though these hallmarks were initially established in predominantly male samples [Exner et al., 2008]. Indeed, earlier work in small samples $(\mathrm{N}<100)$ reported larger ventricles and smaller volumes in the frontal [Narr et al., 2001] and temporal [Bryant et al., 1999; Narr et al., 2001] lobes in SCZ males than females, and greater volume reductions [Takayanagi 
et al., 2011] and leftward asymmetry in the amygdala [Niu et al., 2004]. Other small-scale studies reported smaller ACC and insula volumes in SCZ females compared to healthy controls [Duggal et al., 2005; Goldstein et al., 2002; Takahashi et al., 2002], but differences were not significant in males. More recently, Womer et al. [Womer et al., 2016] revealed smaller cerebellar vermis volume in a matched sample of male SCZ patients $\left(n=24, M_{a g e}=31.0 \pm 10.6\right)$ compared to male controls $\left(n=24, M_{a g e}=33.7 \pm 8.9\right)$, but no significant effect in females $\left(n_{s c z}=26, M_{a g e}=30.7 \pm 10.5\right.$; $\mathrm{n}_{\text {Controls }}=30, \mathrm{M}_{\mathrm{age}}=32.0 \pm 12.0$ ). Du Plessis et al. [du Plessis et al., 2020] identified a 3-way interaction between sex, first episode SCZ, and childhood trauma on hippocampal subfield volumes extracted with the FreeSurfer-v.6.0 hippocampal subfield protocol [Iglesias et al., 2015], such that female SCZ patients $(n=21)$ had a larger hippocampal fissure than female controls $(n=35)$, male SCZ patients $(n=58)$, and male controls $(n=47)$ who were matched for age $\left(M_{\text {age }}=\right.$ $23 \pm 7$ ). However, further work is needed to determine the reliability of these findings given the small sample size and the fact that the fissure is prone to noise given its small size and proximity to the ventricles.

White matter microstructure is also impacted in SCZ, potentially in a sex-specific manner. Recently, Kelly et al. [Kelly et al., 2018] conducted the largest-ever meta-analysis of white matter diffusion in 1,963 SCZ patients (ages 18-86 years) and 2,386 controls (ages 18-77 years) on behalf of the ENIGMA-SCZ Working Group. Main analyses revealed a widespread pattern of lower FA in SCZ patients, but sex-by-diagnosis interactions were not detected. Sex-specific analyses, however, revealed lower FA in SCZ females $(n=671)$ than female controls $(n=1,090)$ in 20 of 25 white matter tracts, whereas lower FA in SCZ males $(n=1,292)$ was only observed in 14 tracts compared to male controls $(n=1,296)$; female-specific significant effects were observed in the superior corona radiata, uncinate fasciculus, IFOF, hippocampal segment of the cingulate gyrus, and internal capsule. Similar to Kelly et al. [Kelly et al., 2018], other work by the ENIGMASCZ Working Group [van Erp et al., 2018] revealed non-significant sex-by-diagnosis interactions on cortical thickness or surface area, but sex-specific analyses were not tested. 
Recently, machine learning methods were used to identify voxel-based neuroimaging phenotypes across 157 SCZ patients ([van Erp et al., 2018], 24.8\% female) and 169 controls ([van Erp et al., 2018], 31.4\% female) when the diagnostic label was hidden [Honnorat et al., 2019]. A semi-supervised clustering method (CHIMERA) revealed three distinct clusters: clusters 1 and 2 consisted of more than $80 \%$ males, but cluster $3(n=52)$ had a mixed sex distribution ( $56 \%$ male). Pairwise comparisons revealed less CSF expansion and lower white matter, total brain, and intracranial volumes in cluster 3 compared to other clusters, with less frontal and temporal atrophy [Honnorat et al., 2019]. While these methods need to be tested in larger samples, results appear to align with findings from Kelly et al. [Kelly et al., 2018], Bryant et al. [Bryant et al., 1999], and Narr et al. [Narr et al., 2001], suggesting greater white matter disruption with relatively preserved frontal-temporal gray matter in female SCZ patients. It is unclear whether this phenotype is a cause or consequence of SCZ.

\section{Substance Use Disorders}

Prevalence rates of substance use disorders (SUDs) tend to be higher in males for most substances, but sex differences in overall SUD prevalence is narrowing as the number of females with SUDs increases [Keyes et al., 2008; McHugh et al., 2018; Seedat et al., 2009]. While prevalence rates for SUDs are generally higher in males, the health impact of SUDs is more detrimental in females for most (but not all) substances of abuse and dependence [McHugh et al., 2018]. Specifically, females with SUDs have higher risk of alcohol-induced injuries, liver damage, tobacco-induced heart and lung disease, and increased mortality [Agabio et al., 2016]. Females also show greater negative side effects from marijuana, cocaine, and heroin use, and metabolize nicotine more quickly than males. Among males and females who consume equal amounts of alcohol, females show increased risk for alcohol toxicity, cognitive impairment, sleep disturbance, and intoxication [Franconi and Campesi, 2014]. 
Several papers describe sex differences across all phases of substance use and addiction [Becker and Hu, 2008; Bobzean et al., 2014; Keyes et al., 2008; McClellan et al., 2017]. McClellan et al. [McClellan et al., 2017] recently detailed faster escalation of drug use, maintenance of drug use at higher doses, greater negative affect and stress during withdrawal, and greater likelihood of relapse in females. These differences may be due to a sexually dimorphic reward system that differentially reinforces substance use behaviors between females and males [Fattore et al., 2014]. Accordingly, motivations for substance use also differ by sex, with females engaging in substance use to reduce stress and negative emotions, and males engaging in substance use to increase positive emotions [Glavak Tkalić et al., 2013]. In studies of tobacco use, females show faster nicotine metabolism than males, which is linked to higher levels of estrogen and the metabolic liver enzyme cytochrome P450 2A6 (CYP2A6) [Benowitz et al., 2006; Franconi et al., 2012]. Additionally, women taking oral contraceptives, particularly estrogen-only, show faster nicotine clearance than women on progesterone-only contraceptives or not taking any contraceptives [Benowitz et al., 2006]. Interestingly, sex differences in nicotine clearance do not exist between men and post-menopausal women, suggesting an important and specific role of estrogen in nicotine metabolism. Similar to AUD, women exhibit greater health consequences of nicotine than men, specifically in risk for coronary heart and lung disease [Greenfield et al., 2010; Huxley and Woodward, 2011].

Treatment-seeking behaviors and treatment success for SUDs also differ by sex. A report from the Substance Abuse and Mental Health Services Administration [Substance Abuse and Mental Health Services Administration, 2016] showed that significantly more males than females were admitted to a treatment facility for misuse of alcohol and marijuana ( $72 \%$ males), heroin (66\% males), and non-smoked cocaine (69\% males). By contrast, admissions for misuse of crack, methamphetamines/amphetamines, and opiates other than heroin were more proportional between sexes [Substance Abuse and Mental Health Services Administration, 2016]. Interestingly, $82 \%$ of admissions for sedative misuse (barbiturates, benzodiazepines, etc.) were 
among white, non-Hispanics, with significantly higher admissions among females $(50 \%)$ than males $(32 \%)$. This is perhaps not surprising as benzodiazepines are the hallmark treatment for anxiety disorders, which are significantly more common in females than males. Indeed, a recent study of benzodiazepine use and misuse in 349 adults $\left(M_{\text {age }}=39.2 \pm 13.0\right)$ revealed a lifetime benzodiazepine prescription in $58 \%$ of females compared to $44 \%$ of males $(p<0.01)$ [McHugh et al., 2021]. While the proportion of individuals who misused these prescriptions did not differ significantly by sex, $68 \%$ of females (vs. $49 \%$ of males) attributed prescription misuse to coping motives, whereas $40 \%$ of males (vs. $19 \%$ of females) attributed misuse to drug enhancement. Further, females with a lifetime history of benzodiazepine misuse reported significantly higher levels of drug craving compared to males [McHugh et al., 2021].

The neuroimaging literature on sex differences in SUDs is complicated, as brain signatures differ based on the substance of abuse/dependence. In individuals with cocaine dependence, Rando et al. [Rando et al., 2013] used VBM to identify sex differences in gray matter volume in cocaine-dependent individuals who were completing an in-patient treatment program and had been abstinent at least 3 weeks prior to the imaging scan $(\mathrm{N}=36)$ compared to controls. Results revealed lower gray matter volume in the left inferior frontal gyrus, insula, STG, and hippocampus of cocaine-dependent females $\left(n=18, M_{\text {age }}=36.7 \pm 5.6\right)$ compared to female controls $\left(n=22, M_{a g e}=31.5 \pm 8.3\right)$, whereas cocaine-dependent males $\left(n=18, M_{a g e}=38.2 \pm 5.4\right)$ had lower gray matter volume in the precentral gyrus and mid-cingulate compared to male controls $(n=28$, $\left.M_{\text {age }}=30.9 \pm 9.7\right)$. More recently, the ENIGMA-Addiction Working Group revealed significantly lower gray matter volume in the left anterior insula and lingual gyrus in cocaine-dependent females $\left(n=70, M_{a g e}=39.6 \pm 7.6\right)$ compared to female controls without a history of substance dependence or other disorders $\left(n=70, M_{a g e}=37.2 \pm 9.8\right)$, and after adjusting for age, education, project site, and ICV [Rabin et al., 2020]. There were no significant differences between cocaine-dependent males $\left(n=140, M_{a g e}=37.8 \pm 6.7\right)$ and male controls $\left(n=140, M_{a g e}=37.0 \pm 8.5\right)$, but within-group analyses showed that hippocampal volume was negatively associated with the duration of cocaine use in 
cocaine-dependent males, but not in cocaine-dependent females. These results did not change when adjusting for current tobacco use and alcohol use disorder.

Problematic alcohol use is also associated with sex differences in microstructural white matter and substructures of the limbic system. A study of 303 heavy alcohol users (ages 21-56, $30 \%$ female) used DTI to determine the effect of problematic alcohol use and alcohol drinking frequency on five white matter tracts (CC, fornix, external capsule, superior longitudinal fasciculus (SLF), cingulum) that were previously associated with heavy drinking (here defined as $\geq 4$ heavy drinking episodes per month in females and $\geq 5$ episodes per month in males) [Monnig et al., 2015]. The authors used confirmatory factor analysis and structural equation modeling to create a latent "white matter factor" based on FA values of the five tracts of interest. Sex significantly moderated the effect of the proportion of drinks per day on the white matter factor, such that a higher number of drinks per day was associated with lower FA in the white matter factor in females, but not males [Monnig et al., 2015]. Interestingly, a more recent voxelwise study of the effects of heavy alcohol use history on white matter microstructure revealed a somewhat different pattern of effects by sex ( $\mathrm{N}=90$, ages $23-76$ years) in abstinent adults who met DSM-IV criteria for lifetime alcohol use or dependence disorder (minimum heavy drinking history of 5 years). Specifically, alcoholic males who were abstinent at the time of scan $(n=23)$ had lower FA than non-alcoholic male controls in the CC, SLF, arcuate fasciculus and external capsule, whereas abstinent alcoholic females $(n=26)$ had higher FA in these regions compared to female controls [Sawyer et al., 2018]. While these results seem inconsistent with those by Monning et al. [Monnig et al., 2015], these studies cannot be truly compared given that participants in Sawyer et al. [Sawyer et al., 2018] had been sober for an average of 4-9 years whereas participants in the former study were not abstinent. Further, although both studies focused on the same tracts of interest, a direct comparison cannot be made between tract-specific FA outcomes and an aggregate measure of tract FA (i.e., white matter factor) as examined in Monning et al. [Monnig et al., 2015]. Several other methodological components may have also contributed to the 
seemingly divergent study outcomes including differences in sample size and composition, operational definitions for 'heavy drinking', duration of heavy drinking etc.), highlighting a few of the many challenges.

A recent substructural analysis $(\mathrm{N}=131$, ages $23-76$ years, $46.9 \%$ female) from the same group showed that a longer duration of alcohol sobriety predicted larger volumes in the hippocampal CA1 region for males, but smaller CA1 volumes in females [Sawyer et al., 2020]. Finally, the most recent work from ENIGMA-Addiction examined neuroimaging sex differences in the effect of alcohol dependence on subfield volumes of the hippocampus and amygdala [Grace et al., 2020]. Subfields were identically processed and QC'd across 10 cohorts using the FreeSurfer-v.6.0 pipeline for 643 individuals with alcohol dependence (35\% female) and 323 healthy controls (30.3\% female) without alcohol dependence or other psychiatric disorders. Results revealed significant main effects of sex and sex-by-diagnosis interactions in a priori ROI of the whole bilateral amygdala and basolateral subregion, but not the central amygdala. Specifically, alcohol-dependent males $(n=418)$ had $5 \%$ and $3 \%$ lower volumes in these regions (respectively) compared to male controls $(n=225)$ after adjusting for age, education, ICV, and tobacco use. These regions did not differ significantly between female cases $(n=225)$ and controls $(n=98)$. An exploratory analysis of sex differences in the accessory basal, anterior and corticoamygdaloid nuclei also revealed significant sex-by-diagnosis interactions, with larger volumes in males than females with alcohol dependence. Sex-by-diagnosis interactions in hippocampal subfields were not significant in any a priori defined region (CA1, CA3, subiculum, dentate gyrus), but analyses of exploratory subfields revealed significant interactions in the bilateral hippocampalamygdala-transition-area (HATA) and right fimbria, with $11 \%$ and $8 \%$ lower volumes in alcohol dependent males than females, respectively, after adjusting for the same covariates [Grace et al., 2020].

Finally, preliminary work from the ENIGMA-Addiction Working Group showed a significant role of sex on the effects of cannabis dependence $\left(\mathrm{N}=270,31.1 \%\right.$ female, $\left.\mathrm{M}_{\mathrm{age}}=26.91 \pm 9.66\right)$ in 
the cerebellum and OFC [Rossetti et al., 2019]. Specifically, females with cannabis dependence showed smaller white matter volume in the cerebellum and a thinner OFC compared to female non-addicted cannabis users and female controls. Cannabis use status was not associated with imaging outcomes in males, but males with higher monthly cannabis use and earlier cannabis use onset had lower cerebellar gray and white matter volume, respectively [Rossetti et al., 2019]. Other studies have identified sex differences in neural circuitries underlying SUDs using resting state functional connectivity, and these have been reviewed elsewhere [Hamidullah et al., 2020; Rakesh et al., 2020].

Sex differences in comorbid and multimorbid SUD profiles further complicate the interpretation of sex effects on the brain, particularly for substances of abuse/dependence with opposing mechanisms of action or prescription medications designed to treat a separate psychiatric condition. Indeed, McCabe et al. [McCabe et al., 2017] showed that most past-year non-alcohol SUDs co-occurred with another SUD, with a greater likelihood of multiple SUDs in males than females. These factors illustrate the complexities of SUD research, and future neuroimaging studies may benefit from the use of sophisticated machine learning algorithms in large and diverse cohorts, to model these complex interactions with precision.

\section{Sex Differences in Neurodegenerative Disorders}

The role of biological sex in neuropsychiatric conditions is relevant for understanding the trajectory of brain health across the lifespan, as all of the abovementioned conditions have been linked to an "accelerated" profile of aging using neuroimaging [Hajek et al., 2019; Han et al., 2019; Koutsouleris et al., 2014; Van Gestel et al., 2019] and epigenetics [Fries et al., 2017; Luo et al., 2020; Wolf et al., 2017]. While these studies use algorithmic tools to determine patterns that appear consistent with "accelerated aging", it is speculative at best to conclude that biological patterns that appear older than expected for a person's chronological age are representative of true biological aging - a phenotypic concept that has no true measurement [Butler et al., 2020; 
Freund, 2019]. Still, each of the psychiatric conditions discussed in this review has been linked to increased risk for dementia, suggesting an important role for mental distress as an etiological predictor of neurodegenerative disease. In the sections below we describe the most common neurodegenerative conditions and review the existing evidence for sex effects on disease prevalence, symptom patterns, and neuroimaging signatures. As with the previous section, conditions are presented in order of the magnitude of the observed sex difference in disease prevalence.

\section{Parkinson's disease}

Parkinson's Disease (PD) is a neurodegenerative movement disorder characterized by symptoms of resting tremor, bradykinesia, rigidity, gait and speech abnormalities; cognitive systems are also affected [Podcasy and Epperson, 2016]. Classic biomarkers of PD include loss of dopaminergic neurons in the substantia nigra and basal ganglia, as well as cell loss in the nucleus basalis of Meynert - the major cholinergic projection to the cortex [Benazzouz et al., 2014; Zarow et al., 2003]. The first-line treatment for PD includes levodopa (L-DOPA) or carbidopa, which promote dopamine synthesis from tyrosine. Unfortunately, an estimated $50-70 \%$ of dopaminergic neurons are lost by the time a diagnosis is made, reducing the potential efficacy of pharmacotherapies [Cheng et al., 2010].

The prevalence of PD is higher in males and typically diagnosed two years earlier than in females [Haaxma et al., 2007; Taylor et al., 2007]. Males with PD report more sleep disruption, sexual dysfunction, urinary incontinence, and symmetrical upper body symptoms than women. Males also exhibit more severe cognitive impairment including emotion recognition compared to women, and are more likely to progress to dementia [Cerri et al., 2019; Cholerton et al., 2018]. Female-dominant symptoms include greater postural problems and poorer motor performance on the Unified Parkinson's Disease Rating Scale (UPDRS) [Cerri et al., 2019; Fahn et al., 2008] - a common measure of clinical function in PD patients. Females also tend to report more non-motor 
symptoms than males, including increased fatigue, apathy, cardiovascular symptoms, anhedonia, sensory dysfunction, constipation, sweating, and pain [Martinez-Martin et al., 2012; Solla et al., 2012].

Across all patients with PD, neuroimaging studies show lower gray matter volume in the basal ganglia, motor cortex, and cerebellum and lower FA in the substantia nigra of PD patients compared with controls [Berman and Miller-Patterson, 2019; Geng et al., 2006; Kang et al., 2015; Zhang et al., 2015]. In recent work by the ENIGMA-Parkinson's Disease Working Group, individuals with PD ( $n=2,367, M_{a g e}=63.4 \pm 9.8,36 \%$ female) showed widespread cortical thinning in parietal association areas, primary and supplementary motor areas, inferior temporal cortex, precuneus, and PCC, and smaller volumes in the bilateral putamen, hippocampus, amygdala and accumbens compared to healthy controls ( $n=1,183, M_{\text {age }}=59.4 \pm 12.3,46 \%$ female) [Laansma et al., 2020], but sex-by-diagnosis interactions were not significant.

Despite reported sex differences in PD clinical presentation and symptom expression, there is almost no literature devoted to sex differences in structural brain metrics in PD. In one of the only studies we could find, Tremblay et al. [Tremblay et al., 2020] used DTI and deformationbased morphometry (DBM - an alternative to VBM that maps regional atrophy patterns and has heightened sensitivity to subcortical brain atrophy [Scanlon et al., 2011]) - to examine sex-specific brain atrophy maps and structural connectivity patterns between 232 PD patients (36\% female) and 117 healthy controls (33\% female) participating in the Parkinson's Progression Markers Initiative (PPMI). "W-score" (covariate adjusted Z-scores) maps [Jack et al., 1997; La Joie et al., 2012] were created to obtain cross-sectional estimates of regional brain atrophy relative to controls after accounting for age and sex. Here, the W-score indicated the normal deviation in a PD patient's brain metric relative to the expected value in controls. A total of 246 cortical and subcortical regions were analyzed using the parcellation from the Brainnetome atlas [Fan et al., 2016]. Results showed lower cortical gray matter volume and higher intracerebral CSF in females than males with PD after adjusting for ICV. There were no sex differences in cortical thickness, 
but DBM analyses revealed greater brain atrophy overall in males than females with PD, despite comparable levels of disease severity and duration (mean age of onset was 63.9 years for both sexes). Regionally, PD males had a stronger pattern of atrophy in frontal-subcortical structures (thalamus, two regions of the left insula, six regions of the frontal lobe) compared to PD females, whereas PD females had a stronger atrophy pattern in the posterior cortex (superior parietal, right occipital lobe) and three regions of the frontal cortex. Structural connectivity analyses also showed lower local network efficiency in males than females with PD in $\sim 45 \%$ of the 246 ROls, and efficiency in two regions was associated with cognitive performance in males only. Specifically, local efficiency in the right IFG was positively associated with delayed recall on the Hopkins Verbal Learning Test (HVLT), and efficiency in the ventral agranular insula was positively associated with scores on the Letter Number Sequencing task ( $r$ 's=0.42). DBM measures were not associated with clinical or cognitive outcomes in males or females.

The major takeaway of the abovementioned study was that males with PD had greater brain disruption than females, with more widespread sex differences in local white matter network efficiency than brain atrophy. Lower network efficiency indicates less efficient information transfer between white matter networks [Bullmore and Sporns, 2009; Sporns, 2018], and has been recently linked to age-related cognitive difficulties on tests of memory, executive function, and attention - domains that are tapped by the HVLT and LNS. As several studies show that microstructural white matter degeneration precedes brain atrophy in normal aging, the degree to which local efficiency metrics are a marker of sex differences in PD versus normal aging are unclear, even with the use of W-scores. Further, the beta weights for each of the significant sex effects appeared to be stronger in DBM (absolute $\beta$ 's=0.39-0.72) than connectivity (absolute $\beta$ 's $<0.001)$ metrics. While these differences may be due to a lack of standardized beta scaling, these methods are not described and the effect sizes were not interpreted [Tremblay et al., 2020].

Other DTI work in the PPMI cohort [Burciu et al., 2017] has shown that longitudinal changes in the degree of free water in the posterior substantia nigra (PSN) is a marker of PD 
progression using Pasternak's free water method [Pasternak et al., 2009], and that sex is a strong predictor of PSN free water over a 4-year period (Mage at baseline=59.1 \pm 9.7 ). Specifically, the 4year change in PSN free water was significantly greater in males $(n=34)$ than females $(n=12)$ with PD, and annual changes in PSN free water were significantly associated with disease progression on the widely used Hoehn and Yahr scale. However, these results should be interpreted with caution given the very small sample size and imbalanced sex ratio in this study [Burciu et al., 2017].

\section{Multiple Sclerosis}

Multiple sclerosis (MS) is a chronic and debilitating neuroinflammatory condition that affects approximately 2.8 million people worldwide [Hauer et al., 2020]. MS is the most common neuroimmune condition in young adults, with a diagnosis typically occurring between ages 20-50, and average onset around age 32 in high-income countries of North America, Western Europe, and Australia [Wallin et al., 2019; Walton et al., 2020] [Hauer et al., 2020]. MS is twice as common in females than males worldwide, with regional sex ratios as high as 4:1 [Walton et al., 2020]. In the United States, females account for over $70 \%$ of the 409,217 MS cases that were reported in 2019 [Institute for Health Metrics and Evaluation (IHME), 2019].

MS consists of four primary clinical subtypes. Relapsing-remitting MS (RRMS) is the most common MS subtype ( $85 \%$ of cases) and is characterized by intermittent neurologic symptoms (relapse) followed by partial or complete remission [Luchetti et al., 2018]. Secondary progressive MS (SPMS) is the second most common form of MS that develops after an initial period of RRMS (often during the fifth decade) and is characterized by chronic worsening of neurologic symptoms [Tutuncu et al., 2013]. Primary progressive MS (PPMS) is the most aggressive form of MS and characterized by chronic worsening of symptoms from the point of onset [Kantarci, 2019]. Finally, progressive-relapsing MS (PRMS) is the least common form of MS, in which patients experience chronic worsening of symptoms punctuated by periods of remission [Kalincik et al., 2013]. RRMS 
is significantly more common in females than males (risk ratio=1.08), with a $17.7 \%$ higher relapse rate in females [Kalincik et al., 2013]. By contrast, males typically exhibit more severe clinical symptoms, faster disease progression, and younger age of conversion from RRMS to SPMS than females [Golden and Voskuhl, 2017]. Sex differences in the prevalence of PPMS have not been reported, but male sex is a significant predictor of PPMS following a radiologically isolated syndrome (i.e., asymptomatic white matter lesions) [Kantarci et al., 2016b].

The neuropathological footprint of MS is heterogeneous across individuals, but traditional characteristics include neuroinflammation, enlarged ventricles, whole brain atrophy and regional atrophy of the medial temporal lobe, thalamus, and deep gray matter, and demyelinating lesions that typically affect white matter in the optic nerve, brainstem, basal ganglia, spinal cord, and fiber tracts proximal to the lateral ventricles [Cortese et al., 2019; Filippi et al., 2019; Lassmann et al., 2007; Reynolds et al., 2011]. Cortical lesions are also detected in over $70 \%$ of patients with SPMS and over $60 \%$ of patients with RRMS [Calabrese et al., 2010]. Neuroimaging is essential for visualizing MS brain abnormalities in vivo. As MS has historically been considered a white matter disease, we were surprised to find that only two studies had examined sex differences in white matter microstructure using DTI. In the earlier of these two studies, Schoonheim et al. [Schoonheim et al., 2014] examined the relationship between voxelwise DTI metrics (FA, mean (MD), radial (RD), and axial (AxD) diffusivity) and cognitive performance in $131 \mathrm{MS}$ patients (67.2\% female) approximately 6 years after diagnosis and 49 age-matched healthy controls (59.2\% female). Most participants had RRMS ( $n=114,79$ females), followed by SPMS ( $n=9,6$ females) and PPMS ( $n=8,2$ females), but subtype was not included in the statistical model. After adjusting for age and education, a generalized linear regression showed significantly lower FA, and higher $\mathrm{MD}, \mathrm{AxD}$, and $\mathrm{RD}$ in the $\mathrm{CC}$, temporal white matter, and posterior periventricular regions in both male and female patients compared to controls. Male patients also had lower FA and higher diffusivity in the posterior $\mathrm{CC}$, thalamus, cerebellum, pons, and fronto-parietal white matter compared to male controls. Case-control differences in voxelwise DTI metrics were larger 
and more expansive in male than female patients, and male patients performed worse on a series of cognitive tasks compared to controls and female patients, but no significant case-control differences in cognitive performance were observed in females [Schoonheim et al., 2014]. A longitudinal study by Klistorner et al. [Klistorner et al., 2018] used DTI, T1-, and T2-weighted scans to examine microstructural changes in brain lesions over an average of 3.5 years (range $=36-50$ months) in 43 consecutive RRMS patients (no relapse; $55.8 \%$ female, $M_{\text {age }}=42.1 \pm$ $6.1)$ and 20 controls $\left(60 \%\right.$ female, $\left.M_{\text {age }}=41.0 \pm 9.1\right)$. Results showed worsening of white matter microstructure within the lesion core of all participants over time, as well as increased lesion volume across the whole brain and increased volume in the lateral ventricle. A significant sex difference was observed for the magnitude of MD change in the lesion core, with males showing an increase in MD nearly twice as large as that in females at the follow-up visit [Klistorner et al., 2018]. Taken together, these two studies align with the clinical and neuropathological literature showing greater disease severity and progression in males than females with MS on indices of white matter microstructure. Given all that is known about the neuroimmunology of MS and the vulnerability of white matter microstructure to inflammation, there is an urgent need for additional DTI and beyond-tensor studies of sex differences in white matter microstructure in MS, particulary in large samples within and across MS subtypes.

While the DTI literature on MS sex differences is sparse, several structural MRI studies have examined sex differences in volumetry and atrophy patterns in MS patients. In an earlier cross-sectional study of subcortical and lesion volume differences between 120 RRMS patients and 50 healthy controls [Schoonheim et al., 2012], male $\left(n=40, M_{\text {age }}=40.4 \pm 9.0\right)$ and female $(n=80$, $M_{\text {age }}=39.6 \pm 8.3$ ) patients exhibited lower volumes in all subcortical regions except the bilateral hippocampus in males, and left hippocampus and bilateral putamen in females when compared to controls. Within the patient group, males had significantly lower volumes than females in the bilateral caudate and putamen, with typically larger effect sizes than females. Lesion volumes did not differ significantly between male and female patients, but in male patients only, larger lesion 
volume was associated with lower cognitive performance on test scores averaged across 7 domains (executive functioning, verbal memory, processing speed, visuospatial memory, working memory, attention, psychomotor speed). More recent cross-sectional work using VBM, however, showed a somewhat different pattern of sex effects between patients and controls in subcortical volumes [Sanchis-Segura et al., 2016; Voskuhl et al., 2020]. Similar to Schoonheim et al. [Schoonheim et al., 2012], Voskuhl et al. 2020 [Voskuhl et al., 2020] (79 RRMS, 10 SPMS, 45 controls, ages 18-69) and Sanchis-Segura et al. 2016 [Sanchis-Segura et al., 2016] (56 RRMS, 63 controls, ages 18-61) reported smaller thalamus volumes in both male and female MS patients compared to controls, as well as smaller putamen volumes specifically in male patients than male controls. In contrast to Schoonheim et al. [Schoonheim et al., 2012], case-control differences in other subcortical structures were not detected. Surprisingly, neither Voskuhl et al. [Voskuhl et al., 2020] nor Sanchis-Segura et al. [Sanchis-Segura et al., 2016] offered any potential explanation for these discrepant studies despite the very limited number of MS studies that have examined sex effecs.

Longitudinal studies have also revealed sex differences in atrophy patterns in MS patients. Specifically, Rojas et al. [Rojas et al., 2013] compared sex differences in total and regional brain volumes, lesion load, and changes in brain volume over a 6 -year period in 45 RRMS patients who were within 60 days of their first demyelinating episode at the baseline visit. Atrophy patterns were measured using the SIENA software from FSL (https://fsl.fmrib.ox.ac.uk/fsl/fslwiki/SIENA) [Smith et al., 2002]. Sex differences in imaging outcomes were not significant at baseline, but after 6 years, males $\left(n=20, M_{\text {age }}=34.2 \pm 1.1\right)$ showed a sharper decrease in TBV and total gray matter and sharper increase in lesion volume and annual percentage of global brain atrophy compared to females $\left(n=25, M_{a g e}=33.5 \pm 1.6\right)$. Sex differences in the distribution of atrophy patterns were also significant, with males showing a more diffuse global atrophy pattern than females, and females showing more localized atrophy in subcortical frontal lobe areas than males [Rojas et al., 2013]. 
Finally, sex differences in neuroimaging phenotypes of MS also differ by age, as older age is linked to greater disease severity and disability burden [Zeydan and Kantarci, 2020]. In a recent lifespan study of brain volume trajectories of $2,199 \mathrm{MS}$ patients $\left(75.1 \%\right.$ female, $\left.\mathrm{M}_{\text {age }}=46 \pm 11.6\right)$, significant age-by-sex interactions were observed for volumes in the lateral ventricle and total normalized gray matter (adjusted for head size using FSL's SEINAX). Post hoc analyses conducted in 10-year age bands (ranging from 18-60+years) revealed significantly lower lateral ventricle volumes in female than male MS patients specifically between ages 40-60, whereas gray matter volumes were lower in male than female patients between ages $18-59$, but not after age 60 [Jakimovski et al., 2020]. The lack of significant sex differences after age 60 are consistent with many other studies discussed in this review, and may be driven by worsening of symptoms in females due to estrogen loss at menopause. Of note, this sample consisted of both RRMS $(n=1,554)$ and PPMS ( $n=453)$ patients, as well as 192 individuals who had been diagnosed with a clinically isolated syndrome (CIS) - a single episode of MS-like CNS inflammatory and demyelinating symptoms that often precedes the development of MS [Miller et al., 2012]. As CIS is not typically defined as an MS subtype in clinical research, direct comparison of results to other studies should be interpreted with caution. Indeed, exploratory statistical models that included MS subtype as a covariate predictor of imaging outcomes were significant, but post hoc analyses by subtype were not reported [Jakimovski et al., 2020]. However, an earlier study conducted by the same group did reveal sex differences in imaging outcomes by MS subtype. Specifically, Antulov et al. [Antulov et al., 2009] reported significantly smaller volumes in normalized peripheral gray matter and larger larger volumes in the lateral ventricle of males $(n=96)$ than females $(n=403)$ with RRMS, whereas females with RRMS had significantly lower normalized white matter volumes than RRMS males. Males with progressive MS subtypes (SPMS, PPMS) also had larger 3rd ventricle widths than females, but results did not survive multiple test correction.

Finally, machine learning may have important utility for defining subgroups of MS patients with shared neuroimaging features that may differ by sex. Indeed, a recent machine learning 
analysis of 8,968 MS patients (RRMS $n=2,884$; SPMS $n=1,837$; PPMS $n=1,601$ ) revealed three primary MRI phenotypes that were distinguished by the temporal sequence of brain abnormalities along the MS disease course [Eshaghi et al., 2019]. (1) cortex-first: most common in RRMS, early atrophy in the occipital, parietal, and frontal cortices and late-stage reduction in T1/T2 ratio in normal appearing white matter (NAWM; an index of extra-lesional white matter changes); (2) NAWM-first: most common in PPMS, early reduction in the T1/T2 ratio in NAWM of the cingulum and CC and late stage atrophy of deep gray matter and the frontal, temporal, and parietal cortices; and (3) lesion-first: most common in SPMS, early and extensive T2 lesions and late stage reduction in the T1/T2 ratio in NAWM. A higher proportion of females than males had RRMS (internal validation dataset: $70 \%$ female, external validation dataset: $61 \%$ female) or SPMS (internal validation dataset: $65 \%$ female, external validation dataset: $67 \%$ female), whereas PPMS was equally common in both sexes. Interestingly, a higher proportion of females than males had the NAWM-first neuroimaging phenotype in the external validation dataset only ( $71 \%$ female) [Eshaghi et al., 2019]. These comparisons were the only analyses completed to address sex effects, but collectively mark an encouraging step towards data-driven discovery of neuroimaging phenotypes that have a sex-specific signature.

Collectively, most of the neuroimaging literature in MS is consistent, generally showing greater imaging abnormalities in males than females, with the exception of white matter indices that tend to be worse in females. Advanced neuroimaging techniques such as quantitative susceptibility mapping (QSM)[Tolaymat et al., 2020], myelin imaging [Ouellette et al., 2020], and diffusion basis spectrum imaging (DBSI) [Shirani et al., 2019] are emerging techniques in the MS literature that provide enhanced biological information relative to conventional structural and diffusion MRI methods. As there are limited discrepancies to address in the existing MS neuroimaging literature, future studies should captalize on the strength of these advanced techniques to better define the biological basis for sex differences in MS and its specific subtypes. 


\section{Alzheimer's disease}

Alzheimer's disease (AD) is the most common dementia syndrome worldwide, affecting approximately 50 million people around the globe, and 6.08 million people in the United States [Brookmeyer et al., 2018; Prince, 2015]. Advanced age is the single greatest risk factor for AD [Alzheimer's Association, 2015; Guerreiro and Bras, 2015], with the majority of AD cases occurring after age 65 (late onset AD). Females have a significantly higher risk for AD than males, representing approximately two thirds of all AD cases [Mielke et al., 2014]. In the United States, the estimated lifetime risk for $A D$ was approximately twice as high in females than males both at age 45 and age 65 [Anon, 2020]. Although higher AD risk in females has been largely attributed to increased longevity, many studies suggest this is an oversimplification of numerous confounding factors that may differentially influence male and female AD risk. For example, the e4 allele of the Apolipoprotein $\mathrm{E}(A P O E)$ gene is an established genetic risk factor for late onset AD; at least one copy of APOE4 is found in over $60 \%$ of AD cases [Farrer et al., 1997; Raber et al., 2004]. Previous work suggests that there may be an interactive effect of APOE4 and sex as it relates to age, where females with one copy of APOE4 have increased risk of developing $A D$ between ages 65-75 compared to males with the same genotype [Neu et al., 2017]; we discuss these interactions in relation to imaging variables in the paragraphs below. Other more controversial factors that may contribute to increased AD risk in females include lifetime estrogen exposure, number of pregnancies, and use of hormone replacement therapy [Georgakis et al., 2016; de Lange et al., 2020; Prince et al., 2018].

The neuropathological substrates of AD include abnormal accumulation of amyloid-beta $(\mathrm{A} \beta)$ and neurofibrillary tau tangles (NFT) that results in neurodegeneration and progressive changes in cognition [Bondi et al., 2017; Jack et al., 2018a]. A $\beta$ and NFT have long been used as part of the neurodiagnostic system of $A D$, but disease classification schemes have evolved considerably in the past 30 years. In general, most of the scientific community agrees that AD refers to a continuum of $A \beta$ and NFT accumulation and neurodegeneration that manifests some 
degree of cognitive impairment, but there are no assumptions about the temporal sequence of these biomarkers or their causal mechanisms [Bondi et al., 2017]. In terms of progression, most studies show that $A D$ neuropathology begins in the brainstem and medial temporal lobe (hippocampus, ERC), affecting storage and retrieval of new information early during the disease course [Bondi et al., 2017; Braak et al., 2011; Braak and Braak, 1991; Braak and Del Tredici, 2015; Hyman et al., 1984]. Eventually AD pathology spreads beyond the MTL to the neocortex and frontal lobes, affecting other cognitive systems such as language and executive function [Ramirez-Gomez et al., 2017]. Widespread brain atrophy is normative in the late stages of AD which typically corresponds to cognitive impairment in multiple domains and the inability to complete basic or instrumental activities of daily living (ADLs) [Jack et al., 2018b].

Although changes in cognitive function are the focus of most $A D$ clinical research, significant changes in mood, behavior, and somatic symptoms accompany and often precede cognitive decline. Sex differences have been reported in many of these symptoms. Specifically, females with $A D$ exhibit greater insomnia, disability, depression, reclusiveness, emotional instability, delusions, and manic symptoms than males with $A D$. Females with $A D$ also tend to demonstrate a classical amnestic cognitive signature and faster progression of clinical symptoms and brain atrophy after a suspected diagnosis compared to males with AD [Hebert et al., 2013; Laws et al., 2016; Sinforiani et al., 2010]. By contrast, males with AD exhibit greater symptoms of apathy, aggression, agitation and socially inappropriate behaviors [Ferretti et al., 2018]. On average, males also exhibit cognitive symptoms at an earlier age, have a shorter disease course, and are more frequently classified by a non-amnestic cognitive phenotype compared to females, despite the presence of AD neuropathology [Liesinger et al., 2018].

Evidence from postmortem studies suggest that males and females exhibit different susceptibilities to AD neuropathology [Braak and Braak, 1991; Serrano-Pozo et al., 2011], particularly in the accumulation of NFT. A neuropathology study in 1028 deceased individuals (AD, $n=736$; non-demented controls, $n=292 ; M_{\text {age }}$ at death $\sim 2$ years) with antemortem clinical 
assessments showed that females had significantly greater NFT density than males in both the $A D$ and control group after adjusting for age and cognitive symptom duration. Further, a significantly higher proportion of AD females $(n=345)$ reached the highest stage of NFT severity (i.e, Braak Stage VI) compared to AD males ( $n=391)$ [Filon et al., 2016]. Similarly, Liesinger et al. [Liesinger et al., 2018] revealed increasingly greater NFT burden with advanced age in the hippocampal CA1 and subiculum in females than males with $A D$ at autopsy, with the largest sex differences in the oldest old (ages 90-99). Beyond the hippocampus, males and females had a slightly different pattern of NFT pathology, such that NFT burden was most pronounced in the hippocampus of $A D$ females and in the neocortex of $A D$ males with relative hippocampal sparing. Beyond the hippocampus, AD females had greater NFT burden than AD males in the association cortex after age 60 . In early-onset AD cases (ages 50-59), males had greater NFT burden in the fronto-parietal cortex than females, but females had around 6 times the NFT burden in the visual cortex [Liesinger et al., 2018]. While these earlier studies provided seminal evidence of sex differences in neuropathology, in vivo examination of $A D$ biomarkers has become the gold standard for assessing AD pathology through CSF analysis of $A \beta$ and tau and/or positron emission tomography (PET) radiotracers that detect amyloid (e.g., Pittsburgh Compound-B $\left({ }^{11} \mathrm{C}\right.$ $\mathrm{PiB}),{ }^{18} \mathrm{~F}$-Florbetapir) and tau ( $\left.{ }^{18} \mathrm{~F}-\mathrm{AV} 1451\right)$ burden in the brain [Leuzy et al., 2019; Maclin et al., 2019; Márquez and Yassa, 2019].

So far, neuroimaging studies of sex differences along the AD continuum generally mirror neuropathological findings. As the hippocampus is one of the earliest targets for AD pathology, several studies have focused on hippocampal morphometry in relation to clinical stages and $A D$ biomarkers $(A \beta$ and tau). In longitudinal work from the Alzheimer's Disease Neuroimaging Initiative (ADNI) cohort, Koran et al. [Koran et al., 2017] examined the impact of sex and AD biomarkers (CSF $A \beta_{42}$, total-tau) on hippocampal volume and cognitive performance in participants with mixed cognitive status (normal $n=348, M C I n=565, A D n=185$ ) at their baseline visit and over an average of 2.5 years (range=0-9 yrs, $M_{\text {age }}$ across all groups at baseline $=72-75$ 
years). Cross-sectional interactions between sex and AD biomarkers were not significant, but longitudinal analyses revealed significant interactions between sex and AD biomarkers on hippocampal atrophy and cognitive decline on executive and memory tasks after adjusting for age, sex, education, diagnosis, ICV, and scanner strength. Specifically, females exhibited a faster rate of hippocampal volume loss and executive decline than males in the presence of high CSF total tau and low CSF $A \beta_{42}$ (an indicator of high $A \beta_{42}$ in the brain). Memory performance also declined faster in females than males with low CSF $A \beta_{42}$, and additional analyses showed that sex effects were most pronounced in females with lower education and the APOE4 genotype [Koran et al., 2017]. In more recent work from the National Alzheimer's Coordinating Center (NACC, N=483, Mage at baseline=70-78 years), a survival analysis showed that hippocampal volume was a significant predictor of progression to $\mathrm{MCl}$ and $\mathrm{AD}$ in females, but not males, regardless of $A D$ biomarker status ( $A D$ biomarkers were not assessed). Specifically, every $1 \%$ increase in hippocampal volume in females was associated with a $46.5 \%$ reduction in the rate of progression to $A D(n=106)$, and a $61.4 \%$ reduction in the rate of progression to $M C l(n=316)$, over a 10-year period [Burke et al., 2019].

Although females generally exhibit greater hippocampal volume loss than males, there may be a stronger link between hippocampal volume and AD pathology in males. For example, recent work using Florbetapir PET to quantify brain amyloid burden in 520 individuals $\left(\mathrm{M}_{\text {age }}=71.3\right.$ $\pm 6.9 ; 178$ cognitively normal, 342 early $\mathrm{MCl}$ ) revealed a negative association between high brain amyloid levels (i.e., amyloid-positivity) and right hippocampal volume in cognitively normal males ( $n=85,18.8 \%$ amyloid + ), but not females $(n=93,36.6 \%$ amyloid +$)$, after adjusting for ICV, age, education, APOE4 carrier status, and performance on the Montreal Cognitive Assessment (MoCA) [Caldwell et al., 2018]. Similar results were observed in the subiculum of the hippocampus in a secondary analysis of hippocampal subfield volumes (parcellated with FreeSurfer-v.6.0). Specifically, amyloid positivity was negatively associated with right subiculum volume in cognitively normal males, but not females. These sex differences yielded tighter confidence 
intervals than those in the whole hippocampus, suggesting sex effects in whole hippocampal volume were driven by underlying effects in the subiculum. Neither whole hippocampal volume nor subfield volumes differed by sex in participants with early $\mathrm{MCl}$, and 3-way sex-by-diagnosisby-amyloid inteactions were not significant in any other subfield. A significant two-way interaction was reported between sex and diagnosis in the right CA1, but post hoc tests were not reported. While these results suggest possible male vulnerability to amyloid burden than females in the prodromal phases of disease, these findings should be interpreted with caution as there were only 16 males who were both amyloid-positive and cognitively normal, compared to 34 females who met this criteria [Caldwell et al., 2018]. Interestingly, this was not mentioned as a limitation of the study.

Studies of cortical thickness and atrophy in regions beyond the hippocampus also show divergent MRI patterns between males and females along the AD continuum. The most consistent of these patterns is faster atrophy in females than males. In a cross-sectional vertex-wide study of cortical thickness in 152 individuals with early stage AD (clinical dementia rating scale $=0.5$ or 1), a hierarchical cluster analysis revealed three dominant patterns of cortical atrophy in $A D$ (medial temporal/cingulate, parietal, diffuse), two of which differed by sex (females $n=101$; males $\mathrm{n}=51$ ) [Noh et al., 2014]. Specifically, a higher proportion of females than males exhibited medial temporal/cingulate dominant atrophy (i.e., $n=52,73.1 \%$ female) or diffuse dominant atrophy (i.e., nearly all association cortices affected except occipital and OFC, $n=72,66.7 \%$ female), whereas the sex ratio for parietal-dominant atrophy (i.e., bilateral parietal cortex, precuneus, DLPFC) was approximately equal ( $n=28,53.6 \%$ female). Individuals with the medial temporal and diffuse patterns were also significantly less educated than those with a parietal pattern, and were more likely to have an APOE4 allele, whereas those with the parietal pattern were significantly younger than the other groups and exhibited the worst cognitive performance [Noh et al., 2014]. Secondary analyses revealed three additional subtypes within the diffuse atrophy group that differed significantly by sex: medial frontal $(n=33,84.8 \%$ female $)$, frontal-parietal $(n=31,58.1 \%$ female), 
and frontal-temporal $(n=8,25.0 \%$ female). The most common pattern in females (medial frontal) was further characterized by greater cortical thickness than the frontal-parietal and frontaltemporal groups, and poorer performance on tests of verbal and visual recognition [Noh et al., 2014]. Although these patterns reflect cross-sectional estimates of sex differences in "atrophy", longitudinal work in early-stage $A D$ patients generally supports the finding of faster cortical thinning in females than males in medial temporal, ACC and frontal areas. Specifically, Lee et al. [Lee et al., 2018] showed modestly faster cortical thinning in females $(n=22$ at baseline, $\left.M_{\text {age }}=68.4 \pm 8.8\right)$ than males $\left(n=14\right.$ at baseline; $\left.M_{a g e}=73.1 \pm 5.6\right)$ over a 5 -year period in the DLPFC, medial PFC, STG, ACC, temporal-parietal and occipital cortices after adjusting for age, education, APOE4 status, disease duration and onset, and ICV. A sex-by-time interaction effect was not significant, but this may be due to the presence of multiple atrophy subtypes, including parietaldominant, which did not differ by sex in Noh et al. [Noh et al., 2014]. Non-significant sex interactions may also be a function of small sample size, which was reduced to only 5 males and 12 females at year 5 due to attrition [Lee et al., 2018]. Of note, there were no significant sex differences in cortical thickness at the baseline visit, which is consistent with cortical thickness estimates in a larger study of 193 AD patients ( $69.4 \%$ female) that did not differ significantly by sex [Seo et al., 2011].

Sex differences in neuroimaging markers also exist in earlier stages of the AD continuum, prior widespread brain atrophy and cognitive impairment. In the ADNI cohort, Hua et al. [Hua et al., 2010] compared male and female brain atrophy rates by disease stage $(A D=144, M C l=338$, cognitively normal=202) over one year with tensor-based morphometry (TBM) - a warping method used to estimate brain tissue shrinkage and expansion. On average, brain atrophy rates were approximately 1-1.5 times faster in females than males, particularly in the medial temporal lobe among individuals with $\mathrm{MCl}$. In another longitudinal study from ADNI, Skup et al. [Skup et al., 2011] revealed significant sex differences in voxelwise atrophy patterns within and across $A D$ diagnostic groups $(A D=197, M C l=266$, cognitively normal=224) in the caudate, thalamus, MTG, 
precuneus, caudate, ERC, and insula over a 2-3 year period after adjusting for ICV, education, sex, age, age ${ }^{2}$, and diagnosis. While the results generally show faster decline in females than males, the directionality and magnitude of these sex differences are not easily interpretable based on the many interactions and post hoc tests reported in the manuscript; we summarize these findings as they were originally reported Table S1 in the Supplementary Material [Skup et al., 2011].

Sex differences in brain white matter also have been reported along the AD continuum, but less has been done in this area and existing findings are less compelling. For example, Salat et al. [Salat et al., 2009] showed smaller white matter volumes in females $(n=54)$ than males $(n=37)$ with $A D\left(M_{a g e}=77.6 \pm 0.7\right)$ in the pars opercularis, OFC, STG, MTG, supramarginal and caudal middle frontal gyri. The authors described the magnitude of these effects as "minor", but effect sizes were not reported in the manuscript. At the microstructural level, a voxelwise DTI study of sex differences in $\mathrm{MCl}$ patients revealed significantly greater white matter disruption (lower FA, higher radial and mean diffusivity) in females $\left(n=21, M_{a g e}=66.2 \pm 6.5\right.$ ) than males $\left(n=12, M_{a g e}=71.3 \pm 7.0\right)$ [O'Dwyer et al., 2012]. Finally, several studies have examined sex differences in the role of white matter lesions in AD progression [Kao et al., 2019; Lee et al., 2016; Nasrabady et al., 2018] since they are present in the majority of the adult population over age 65 [Paul et al., 2005]. White matter lesions are commonly visualized as hyperintense signals (white matter hyperintensities, WMH) on T2-weighted FLAIR scans because the FLAIR sequence suppresses the CSF signal enabling better lesion detection [Kates et al., 1996; Wardlaw et al., 2015]. WMH have been shown to increase risk for AD disease progression in males, but not females. For example, a longitudinal study of $A D$ risk factors showed that severe $\mathrm{WMH}$ volume (>10mm on MRI) surrounding the ventricles (i.e., periventricular) at baseline predicted a nearly 8fold increased risk of progression from $\mathrm{MCl}$ to $\mathrm{AD}$ in males over an average of 13.8 months $(n=101$, hazard ratio $(H R)=7.9,95 \% \mathrm{Cl}[2.4-26.6])$ compared to males with mild to moderate periventricular WMH at baseline [Kim et al., 2015]. Severe WMH in deep subcortical brain tissue 
of males, however, were associated with significantly decreased risk for AD progression $(\mathrm{HR}=0.08,95 \% \mathrm{Cl}[0.02-0.4])$, which was suggested to represent potentially independent mechanisms of periventricular versus deep $\mathrm{WMH}$ in $\mathrm{AD}$ pathogenesis. There was no significant effect of either periventricular or deep WMH in females [Kim et al., 2015]. In an earlier-mentioned study by the NACC, Burke et al. [Burke et al., 2019] showed that WMH risk for AD in males may depend on race and ethnicity, as larger $W M H$ volume predicted faster progression to $A D(H R=4.7$, 95\% Cl [1.1-20.3]) in White males than other race groups over a 10-year period. Cognitively normal males of presumably any race showed a $4.8 \%$ increased risk for progression to $\mathrm{MCl}$ for every $1 \%$ increase in the proportion of $\mathrm{WMH}$ to TBV (hazard ratio $(\mathrm{HR})=1.05,95 \% \mathrm{Cl}[1.01-$ 1.09]); "any race" is presumed because race was not included as a predictor variable in the survival analysis of $\mathrm{MCl}$ for reasons that are not stated. A limitation of this study is the predominantly White Non-Hispanic dataset $(\sim 73.3 \%$ of the total sample $(N=483))$, of which only $14 \%$ of the 105 males who progressed to $A D$ were identified as Black/ African American. WMH did not influence $\mathrm{MCl}$ or $\mathrm{AD}$ progression in females regardless of race or ethnicity [Burke et al., 2019].

An important consideration for future work is that inconsistencies in $A D$ diagnostic methods influence purported sex differences in prevalance, onset, and staging of disease, which have downstream effects on neuroimaging research designs. For example, Liesinger et al. [Liesinger et al., 2018] did not find sex differences in AD prevalence when AD was defined strictly by autopsy-derived neuropathology, but when $A D$ was defined by the a combination of clinical and neuroimaging criteria, females were more likely to present with $A D$ compared to males. Vascular risk factors for $A D$ and comorbidity rates between $A D$ and vascular dementia are important to consider in the context of sex-specific prevalence rates for AD. In a recent populationbased study of dementia-related mortality in Australia, $53 \%$ of dementia-related deaths were documented as "unspecified dementia", 30\% AD dementia, and 12\% vascular dementia (VaD) on death certificates [Buckley et al., 2019]. Death rates attributable to AD were higher in females, 
whereas death rates attributable to $\mathrm{VaD}$ were higher in males. However, prior work from the same group revealed that over half of the females with an antemortem dementia diagnosis did not have dementia listed on their death certificate, and nearly $20 \%$ of females without an antemortem dementia diagnosis had dementia listed on their death certificate [Waller et al., 2017]. These findings highlight reliability issues when using death certificates for retroactive disease classification. Greater prevalence of $A D$ in females may also reflect a survival bias, as $A D$ incidence increases with age and females have longer lifespans than males [Mayeda, 2019]. Accordingly, a neuropathology study of AD patients across six decades revealed an overrepresentation of $A D$ females after age 80 , whereas males were overrepresented between 50-80 years [Liesinger et al., 2018].

\section{Vascular Cognitive Impairment and Dementia}

Vascular cognitive impairment (VCI) is a neurocognitive syndrome caused by occlusive or hemorrhagic damage to the cerebrovascular system that eventually impairs activities of daily living (ADLs) [Paul and Salminen, 2019]. Cerebrovascular damage increases with advanced age due to changes in vessel structure (stenosis, stiffness) and accumulation of atherosclerotic plaque (i.e., vascular aging) that reduces blood flow to the brain [Paul and Salminen, 2019]. Ischemic stroke is a common consequence of cerebral hypoperfusion that accounts for the vast majority $(\sim 87 \%)$ of all stroke syndromes [Benjamin et al., 2017]. The size and the location of vessel damage and infarction are critical predictors of the resulting cognitive phenotype. Accordingly, approximately one third of patients who suffer from a large vessel stroke meet criteria for $\mathrm{VCl}$ within one year of the event [Paul and Salminen, 2019]. However, VCl is a more common consequence of extensive microvascular dysfunction in small vessels of the brain, which supply blood to subcortical brain structures, particularly in the frontal lobe [Akashi et al., 2017]. 
Prior studies have noted greater prevalence of subcortical white matter lesions in females than males, and faster progression of small vessel disease. In a large community sample from Rotterdam ( $N=1,077$, ages $60-90$ yrs, 52\% female), females had a greater incidence of subcortical white matter lesions than males, particularly in frontal and periventricular regions after accounting for other health confounders (diabetes, hypertension, atherosclerosis); subcortical white matter lesions in females were also more severe compared to males [De Leeuw et al., 2001]. A followup study from this group ( $\mathrm{N}=668$, ages $60-90,52 \%$ female) revealed a significantly greater likelihood of subcortical white matter lesion progression $(\mathrm{OR}=1.8)$ and incident cerebral infarctions $(O R=1.63)$ in females than males over a 3-year period [van Dijk Ewoud et al., 2008], aligning with earlier work from the Cardiovascular Health Study $(\mathrm{N}=3,660)$ showing lower likelihood $(\mathrm{OR}=0.74)$ of white matter lacunes in male participants [Longstreth et al., 1998]. A very recent study from the Endovascular Therapy Following Imaging Evaluation for Ischemic Stroke (DEFUSE 3) trial characterized sex differences in cerebrovascular dynamics 6-16 hours after a large vessel occlusion. Here, females ( $\mathrm{N}=90$, median age=72 years) had superior collateral circulation, smaller core lesion volumes, and slower ischemic core lesion growth after large anterior artery occlusions compared to males $(\mathrm{N}=92$, median age $=69$ years $)$. However, females did not improve significantly after intra-arterial medical treatment (while males did improve), and a lower proportion of females (compared to males) achieved functional independence within 90 days [Dula et al., 2020]. Divergent findings between males and females emphasize the importance of sex-specific neurophenotyping, as less severe MRI findings in females may paradoxically translate to significantly worse functional outcomes in females than males.

Less is known about phenotypic sex differences in $\mathrm{VCl}$, and there is essentially no information linking clinical symptoms to specific neuroimaging measures in VCI patients. Of the information we could find, males performed significantly better than females $(N=139$, $M_{\text {age }}=69.3 \pm 12.3,43 \%$ females) on tests of memory 1-month after first-ever stroke, and performed significantly better on tests of psychomotor speed at 1-month and 6-months post-stroke [Rasquin 
et al., 2002]. Males also showed significantly lower likelihood $(O R=0.60)$ of dysfunction in instrumental ADLs in a large clinical trial of lacunar stroke patients $\left(N=2,820, M_{\text {age }}=63.4 \pm 10.8\right.$, $37 \%$ females) [Dhamoon et al., 2015]. However, neither of these studies specifically addressed whether participants met criteria for $\mathrm{VCl}$.

While many studies report higher stroke prevalence in males [Barker-Collo et al., 2015], females exhibit more atypical cardiac symptoms that result in longer delays to seek treatment and a higher rate of misdiagnosis that may contribute to less frequent stroke recovery in females than males [Mayeda, 2019]. Atypical cardiac characteristics in females include non-obstructive cardiac plaques and coronary microvascular dysfunction. Further, females are twice as likely as males to receive a diagnosis of heart failure despite preserved ejection fraction. Clinicians who are unaware of these sex differences, or more typically encounter male patients with classical cardiovascular symptoms, may be less likely to diagnose females with $\mathrm{VCl}$ or $\mathrm{VaD}$ when significant (yet atypical) cardiovascular morbidities are present [Mayeda, 2019].

\section{Frontotemporal dementia}

FTD affects the youngest population of the dementia subtypes on average, with a typical age of onset between $45-65$ years; between $10-15 \%$ of FTD cases are diagnosed before age 50 [Knopman and Roberts, 2011; Onyike and Diehl-Schmid, 2013]. Approximately 50\% of FTD cases show a genetic or familial linkage, making it the second strongest genetically linked condition after autosomal dominant AD. As its name implies, FTD primarily affects the frontal and temporal lobes, with some involvement of the limbic system and striatum [Rohrer, 2012]. Neuropathology of FTD is characterized by prominent microvascular changes and severe astrocytic gliosis. These changes occur in the presence of randomly arranged tau filaments (Pick bodies) in approximately $20 \%$ of FTD cases [Higgins and Mendez, 2000; Lezak et al., 2004]. Over $70 \%$ of FTD cases meet diagnostic criteria for both FTD and AD at autopsy [Chare et al., 2014; 
Lezak et al., 2004; Varma et al., 1999], and typically exhibit focal hippocampal and temporal lobe atrophy. FTD brains that do not resemble the AD phenotype generally show preservation of the acetylcholine system [Huey et al., 2006]. Although the cause of non-genetic idiopathic FTD is unknown, it is more common for individuals with a history of traumatic brain injury and thyroid disease [Kalkonde et al., 2012; Rosso et al., 2003].

FTD consists of several subtypes with distinct symptom profiles, and there is some evidence these subtypes differ by sex. The most common subtype is the behavioral variant FTD (bvFTD) - a dysexecutive syndrome with extreme changes in behavior, judgment, and personality [Piguet et al., 2011]. Here behavioral symptoms often precede cognitive changes and diagnosis by many years. Additional behavioral symptoms include lack of insight and stereotypic behaviors (i.e., purposeless involuntary behaviors that occur without conscious control). Neuropsychological symptoms of FTD include dysnomia and difficulties with confrontation naming, and poor performance on tests of abstraction and reasoning [Cousins and Grossman, 2017; Ranasinghe et al., 2016]. Base rate estimates of bvFTD suggest it is approximately four times more prevalent in males than females, with similar survival rates by sex. In recent work by Ranasinghe et al. [Ranasinghe et al., 2016], females with bvFTD exhibited more delusions and performed more poorly on tests of executive function and visual free recall, whereas males exhibited more apathy, sleep disturbances, and caregiver stress compared to females. This was the only study we could find that examined sex-specific clinical characteristics in bvFTD, and although the sample was larger than many studies of sex differences in neurological disease $(N=204)$, the number of males and females was not given.

Primary progressive aphasia (PPA) is an umbrella term for two FTD subtypes that interfere with the ability to communicate (e.g., semantic fluent PPA, nonfluent agrammatic PPA), and is approximately twice as common in males than females [Mesulam and Weintraub, 1992; Westbury and Bub, 1997]. Although limited work has been done to investigate sex differences in PPA cognitive phenotypes, Rogalski et al. [Rogalski et al., 2007] showed that females with PPA ( $n=40)$ 
performed more poorly than males with PPA $(n=40)$ on tests of category and letter fluency, and females also exhibited greater longitudinal decline than males. While poorer performance in males might be expected given the higher prevalence rate, a study of three university dementia clinics $(\mathrm{N}=353)$ revealed different sex-specific prevalence rates by PPA subtype, with higher male prevalence in semantic PPA (66.7\%) and higher female prevalence in nonfluent PPA $(60.9 \%)$ [Johnson et al., 2005]. In semantic PPA, neuropsychological symptoms are typically characterized by confrontation naming difficulties on the Boston Naming Test, but preserved function on tests of fluency [Marshall et al., 2018]. Sex differences in PPA subtype were not examined in Rogalski et al. [Rogalski et al., 2007], but results align with these findings.

We could not find any neuroimaging study that directly examined sex differences in atrophy patterns in FTD or any FTD subtype. In general, MRI studies of FTD show frontal and temporal lobe atrophy in patients versus controls, with relative sparing of posterior brain regions. However, a recent machine learning study of dementia patients in two European memory clinics $\left(\mathrm{N}=1,213,48 \%\right.$ female, $\mathrm{M}_{\text {age }}=67$ years $)$ showed that only $59 \%$ of FTD patients $(\mathrm{N}=116)$ demonstrated a distinct frontotemporal atrophy pattern; the rest showed a subcortical atrophy pattern similar to AD [Bruun et al., 2019]. Further, across all dementia subtypes (including AD and $\mathrm{VaD}$ ), the highest predictive accuracy was achieved using asymmetric frontotemporal atrophy to distinguish PPA and bvFTD subtypes, and temporal pole volume to distinguish the fluent and nonfluent PPA subtypes (85\% AUC for both analyses) [Bruun et al., 2019]. These findings may reflect sex differences inherent to the male-dominant bvFTD subtype, and a female-dominant nonfluent PPA subtype, but further work is needed to interpret these results.

\section{Dementia with Lewy Bodies}

Lewy body diseases (LBD) refer to a class of syndromes that involve abnormal aggregation of the alpha-synuclein protein (i.e., Lewy bodies) in the brainstem, limbic system, basal ganglia, cortical and olfactory network, and ventral forebrain. This pathology can cause 
motor symptoms, hallucinations, severe functional impairment, and dementia [Mayo and Peavy, 2019]. LBD most commonly refers to Dementia with Lewy Bodies (DLB), PD and PD dementia (PDD), as these conditions share many neuropathological, clinical, and prognostic features [Outeiro et al., 2019]. The overlap in these conditions is significant, with many reporting underdiagnosis of DLB and overdiagnosis of PD. The differential diagnosis of DLB (vs. PDD) is typically based on the development of dementia within one year of motor-related symptoms (i.e., parkinsonism). A distinguishing feature of LBD vs. AD is the higher prevalence and earlier onset in males than females.

DLB is the third most common type of dementia. It accounts for approximately $4-7.5 \%$ of all diagnosed dementia cases [Jones et al., 2014; Kane et al., 2018], but much higher rates of significant DLB pathology have been reported at autopsy [Outeiro et al., 2019]. DLB is often misdiagnosed for other neurodegenerative conditions such as AD [McKeith et al., 2017], and true prevalence rates have been estimated as high as $20-30 \%$ of all dementia cases [Haider et al., 2020]. DLB causes significantly more impairments in activities of daily living (ADLs) compared to other dementias. The clinical profile of DLB includes cognitive fluctuations, visual hallucinations, parkinsonism, and rapid eye movement (REM) sleep behavior disorder, anxiety, depression, and delusions [McKeith et al., 2017]. Visual hallucinations are uniquely prevalent in DLB patients (50$60 \%$ of cases) and occur early in the disease course [Eversfield and Orton, 2019]. Males have approximately four times the incidence rate of DLB compared to females [Savica et al., 2013], though visual hallucinations are reportedly more common in females [Chiu et al., 2018], particularly at older ages and in the context of more severe neuropsychiatric symptoms. Recent work suggests that DLB is more prevalent in females than males after age 75 , which may reflect a survival bias [Mouton et al., 2018]. Sex differences have been less frequently studied in DLB than other LBDs.

Structural MRI studies of DLB patients show lower volumes in the frontal and temporal lobes and insular cortex, with relative sparing of the medial temporal lobe and hippocampus in 
the early stages of disease [Berman and Miller-Patterson, 2019; Oppedal et al., 2019]. Studies using PET and SPECT show hypoperfusion in the occipital cortex and relative sparing of the PCC - a distinguishing feature from AD and potentially PD [Gutman et al., 2018; Ishibashi et al., 2019]. Several studies used DTI to characterize white matter pathology in DLB patients; many show abnormal structural connectivity in visual association areas, but other patterns have been inconsistently defined [Berman and Miller-Patterson, 2019]. However, a literature search for sex differences in neuroimaging characteristics in DLB patients did not yield any results in the past ten years. In early work from members of our group, males with DLB showed gray matter deficits in frontal dorsal and parietal lobes compared to females with $\mathrm{DLB}$, particularly in the $A C C(N=16$, $M_{\text {age }}=76.4 \pm 6.7,50.0 \%$ female) [Ballmaier et al., 2004]. The lack of literature on neuroimaging sex effects in DLB is concerning, particularly as the hallmark feature - visual hallucinations - is reportedly more common in females than males [Chiu et al., 2018].

\section{Using what we know to inform where we go: Addressing remaining gaps}

The studies above highlight the progress and gaps in the neuroimaging literature related to sex differences in health and disease. We propose a number of solutions to the pervasive problems in the existing literature, where many studies are underpowered to detect sex differences in disease, despite large differences in risk factors, co-morbidities, onset, and expression of disease in males and females. In Table 1 we identify several common problems in the neuroimaging literature related to sex differences and offer potential remedies to maximize rigor and reproducibility. We summarize the key messages from Table 1 in the paragraphs below.

1. Relatively few large studies comparing sex differences in MRI outcomes in major brain diseases 
An alarmingly small number of neuroimaging studies have assessed structural brain differences between males and females despite known sex differences in disease prevalence and/or clinical features. This literature is particularly sparse for anxiety disorders, DLB, and FTD, with only one neuroimaging study examining sex effects in $\mathrm{PaD}$, one study in $\mathrm{DLB}$, and no studies in GAD or FTD. There was no clear indication of why such a massive research gap exists, but may be due to a combination of factors stemming from a long history of biases affecting recruitment, retention, and basic design decisions. Additionally, small studies still dominate the neuroimaging literature on sex effects purported sex effects in some conditions are solely based on findings from one or two small studies The risks of small sample sizes have been well documented over the past 20 years and include (but are not limited to) a high propensity for false negatives, false positives, various forms of publication and reporting bias, and inflated effect sizes due to low statistical power [Button et al., 2013; loannidis, 2005a; loannidis, 2005b; loannidis, 2008; Szucs and loannidis, 2017]. Indeed, a review of 41 meta-analyses of brain volume ( $n=461$ unique datasets) revealed an average maximum sample size of 185 per meta-analysis and median statistical power of only $8 \%$ [Button et al., 2013; loannidis, 2011]. As a result, the number of studies that reported a positive result (a nominally significant effect at $\alpha<0.05$ ) was more than double what would be expected based on formal power calculations if all of the reported effect sizes were accurate, and more than 4 times the expected number if only half of the reported effect sizes were accurate. Although sex effects were not analyzed, a separate review of MRI studies examining sex effects between 1980-2007 reported only one study with a male or female $\mathrm{N}>100$ [Cosgrove et al., 2007]. While we applaud these pioneer studies for their clairvoyant recognition of the importance of sex research in the era of MRI, their results must be interpreted with caution until they have been replicated in larger datasets.

An obvious solution to the plague of small sample sizes is to analyze more data, but this may be a catch-22 for investigators without funding to pursue large-scale recruitment. Fortunately, large sample sizes can now be achieved without incurring additional costs by participating in 
consortia such as ENIGMA. Established in 2009, ENIGMA was designed to circumvent the high price of large-scale neuroimaging data collection and capitalize on the massive amounts of archival data that are often untouched after specific research aims are achieved. This global alliance has led the largest-ever neuroimaging studies of several psychiatric and neurological disorders, and created an unprecedented opportunity to perform well-powered, large-scale, investigations of sex differences in neuroimaging across the lifespan. Importantly, ENIGMA is continuously expanding and there is no cost to join (http://enigma.ini.usc.edu/join/). In addition to ENIGMA, other large-scale databases have collected state-of-the-art imaging data and deep phenotyping and are accessible to the research community (e.g., UK Biobank, ADNI, PPMI, HCP, etc.), but these may cost money and/or require a formal application and review process.

\section{2. "Controlling" for sex as a nuisance covariate}

Covarying for sex has become the gold standard in neuroimaging research, but without good reason. The original and appropriate use for covariates is to improve statistical power to detect associations between an independent variable (IV) and the dependent variable (DV) after removing random variance (i.e., noise) associated with the covariate (Cov) [Miller and Chapman, 2001]. In the classical use of ANCOVA, IV should not share any variance with Cov, and the residuals for IV and Cov should be approximately equal (i.e., homoscedasticity of residuals). Further, the relationship between Covand DV should be the same between all levels of IV (i.e., homogeneity of regression slopes) [Lord, 1967]. The assumption of statistical independence between $I V$ and Cov is seemingly not well known by neuroimaging researchers or the greater scientific community at large, as many studies justify the use of a covariate specifically when it differs across levels of the IV [van Eersel et al., 2017]. However, when researchers attempt to "control" for the effect of Cov that is intrinsically related to the IV, covarying for sex may remove variance associated with pre-existing differences in the grouping variable rather than removing 
random variance (i.e., noise) associated with the covariate. This will completely distort the effect of the IV on brain outcomes, rendering results essentially meaningless.

To avoid this outcome we recommend modeling sex-by-diagnosis interactions whenever possible and strongly encourage the reporting of pairwise comparisons even when the interaction term is not significant. For smaller cohorts with limited power, sex-stratified analyses are an acceptable alternative. Low statistical power is the most common reason for non-significant sexby-diagnosis interactions, particularly in psychiatric studies where effect sizes tend to be much smaller than those for neurological conditions [Thompson et al., 2020]. ENIGMA and other very large biobank studies are in a good position to address these issues and test interactions with sufficient statistical power, as well as testing the generalizability and reproducibility of findings across many cohorts and cultures [Palk et al., 2020].

\section{Inconsistent and/or inappropriate methods to correct for head size}

Inconsistent or biased adjustments for head size are a major issue for interpreting the results of prior work. Misinterpretation of neuroimaging sex differences is common when the measure of interest is nonlinearly related to brain size [Brun et al., 2009; Jahanshad and Thompson, 2017; Thompson et al., 2000], and we know that certain parts of the brain do not scale linearly with TBV. Many studies of sex differences in the brain have not correctly modeled allometric scaling. In Luders [Luders et al., 2014], members of our group examined whether sex differences in brain size versus biological sex accounted for apparent sex differences in the morphology of the $\mathrm{CC}$, the major commissure connecting the two brain hemispheres. These analyses showed that the CC was always larger in men than women, but this difference was strongly determined by sex differences in overall brain size. Comparing CC measures between men and women matched for overall brain size may clarify this, as any observed group difference should indicate pure sex effects. Despite many years of work positing profound sex differences in this structure, hardly any callosal differences remained between brain-size matched men and 
women - individual differences in brain size largely accounted for apparent sex differences in corpus callosum anatomy [Luders et al., 2014]. Sex differences in cortical gray matter distribution also depend on brain scale [Luders et al., 2005; Luders et al., 2006]. Variations in methods for head size or ICV correction are found across the literature, and depending on the method used, incomplete adjustment for ICV effects can yield sex differences in regional measures that simply reflect allometric scaling.

Sex differences in head size may also influence artifact measurements on MRI, such as partial volume effects (PVEs). PVEs may occur when there is CSF contamination of white matter voxels or an unclear boundary between gray and white matter tissue. Both of these issues affect the FA signal [Salminen et al., 2016] and are more likely to arise when there is a mixture of different tissue types in a voxel. As the head is larger, on average, in males than females of the same age, males and females scanned at the same spatial resolution (voxel size) and same field of view will tend to yield scans with a greater number of brain voxels in males than females. This means that more voxels will not have partial volume effects in males, and smaller structures will also tend to be more susceptible to partial volume effects in females. As PVEs affect the FA signal, these subtle effects of spatial resolution may mean that FA is lower for people with smaller

brains (in this case females relative to males) even if the microstructural properties were exactly the same. This effect of brain scale on diffusion measures is not commonly modeled, but could be modeled using regression of ICV on the derived measures and comparing corrected and uncorrected results.

\section{Inconsistent inclusion criteria, covariate selection, and risk modeling}

A large part of the crisis of reproducibility in neuroscience stems from the massive variability in study designs. As we described in the sections above, nearly every study in this review used a different set of covariates for analyses of sex effects on MRI measures. Indeed, recent work from Hyatt et al. [Hyatt et al., 2020] reported 37 different covariate sets across 50 
neuroimaging articles. Additionally, simulation analyses showed that the use of different covariate sets significantly altered the observed associations between MRI variables and individual differences in six psychological constructs, sometimes in opposite directions. This variability is a major challenge for interpreting and comparing neuroimaging sex differences from previous studies. To address this we echo the recommendations put forth by Hyatt et al. [Hyatt et al., 2020] to pre-register analyses and justify their use of specific covariates prior to conducting the work.

Separately, many neuroimaging and genetic studies assume sources of risk and comorbidities operate in the same way in males and females despite considerable evidence to the contrary, and these assumptions perpetuate inequalities and disparities in health care [Young and Pfaff, 2014]. It is important to measure and model risk factors that vary by sex, as restrictive inclusion criteria may model the range of disease expression less fully in one sex versus the other, and may inaccurately represent disease effects on the brain. Even if there were no difference in the way a specific brain disease affects males and females, if there are biases in ascertainment, or sex differences in relevant risk factors, or the frequency of co-morbidities (as we note for many disorders above), then these external factors will influence the observed neuroimaging signatrue. By contrast, if there is a fundamental sex difference in how a specific disease is expressed in the brain, then current methods to "correct for" sex differences using a covariate may not yield an accurate representation of the disease, unless the studies are also well powered to test and model interactions.

Ideally, cohort studies should include deeper phenotyping to measure and model diseaserelevant factors that vary by sex, to identify factors that might account for any observed sex difference in disease prevalence or expression. As there is often insufficient power to model interactions, larger multi-cohort studies are needed (along with coordinated analyses such as ENIGMA) as well as deeper phenotyping of individual cohorts. To merge data from diverse cohorts, statistical harmonization efforts may help, as well as efforts to collect common data elements (CDEs) across funded studies, to facilitate adjustments and comparability of findings. 


\section{Lack of dimensional measures for assessing the clinical relevance of sex differences in neuroimaging}

True shifts towards personalized medicine will require a new multilevel biotaxy in which clinical symptoms (including physiology, cognition, affect, and behavior) are mapped to neuroimaging indices and other biomarkers according to age, sex, and ethnicity. This precision can be achieved through big data, machine learning, and other bioinformatics strategies that capitalize on the enormous wealth of existing data in the health sciences fields. Adopting these methods is further motivated by increasing numbers of Requests for Applications by the NIH and other funding agencies that emphasize a need for big data initiatives and secondary analyses of existing data. In addition, the NIMH developed the Research Domain Criteria (RDoC) to bridge the gap from research to clinical care by using a dimensional approach to understand biologically based constructs of human behavior [Insel et al., 2010; Sanislow et al., 2019] These constructs reflect higher-level knowledge of mental function, acquired through integrative models of neuroimaging, neuropsychological assessment, genomics and other omics-based indices of molecular biology (e.g., metabolomics, transcriptomics). Currently there are six RDoC domains: (1) negative valence, (2) positive valence, (3) cognition, (4) social processes, (5) arousal/regulatory systems, and (6) sensorimotor systems. Each domain represents a unique system that is characterized by subconstructs of human function that map onto distinct brain circuits, cells, neurotransmitter patterns, and physiological responses. As such, RDoC is an integrative research strategy designed to capture the dimensionality of the human condition, independent of disease states. One of the benefits of the RDoC approach is cross-disorder synthesis of biological, behavioral, and physiological features, which can then be mapped to neuroimaging. For example, a brief non-scientific survey of the sex-specific clinical differences reported in this review shows a cross-disorder pattern of anxiety and depressed mood and hallucinations and delusions in females, and a cross-disorder pattern of substance misuse and 
risk-taking behaviors in males [Marcus et al., 2008]. Should these phenotypes be confirmed through actual scientific methods, they can be mapped to MRI outcomes to inform a more complete picture of sex effects on the brain regardless of the overarching disease.

\section{Lack of attention to race/ethnicity}

Many studies report health disparities between individuals of different race and ethnic groups [Bell et al., 2018; Jones et al., 2020; Wilkins et al., 2020]. Perhaps the most well-known examples come from the field of cardiology, where ethnic minorities, particularly those who identify as Black/African American, have a significantly higher risk for cardiovascular disease (e.g., hypertension, diabetes, dyslipidemia) than Non-Hispanic Whites [Howard et al., 2017; Howard et al., 2018; Kurian and Cardarelli, 2007; Lepore et al., 2006; Virani et al., 2020; White et al., 2005]. Many other medical disciplines report health disparities by race and ethnicity [Vyas et al., 2020], including the field of neurology. In one recent study of racial differences in $A D$ biomarkers [Morris et al., 2019], Black/African American participants had lower levels of CSF total tau and phosphorylated tau compared to White participants, with stronger differences in Black/African Americans with the APOE4 genotype. Additionally, Black/African American participants with a family history of dementia had significantly lower hippocampal volume than White participants with a family history of dementia [Morris et al., 2019]. In another study on nondemented older adults using Florbetapir PET [Gottesman et al., 2016], Blacks had a more than 2-fold increased risk for elevated amyloid deposition than Whites, which was substantially higher than the risk attributed to female sex (OR 1.7). These two studies represent only a fraction of this emerging literature, but we highlight examples in $A D$ given the higher number of $A D$ studies that also examine sex effects. Indeed, in our review of sex differences in AD neuroimaging markers we described findings from Burke et al. showing that $\mathrm{WMH}$ increased risk for $\mathrm{AD}$ disease 
progression, specifically in Black males [Burke et al., 2019]. Still, most studies have not examined sex-by-race or sex-by-race-by-diagnosis interactions in neuroimaging.

The lack of attention to race and ethnicity in neuroimaging studies is likely due to extremely low representation of minorities in clinical research [Babulal et al., 2019; Canevelli et al., 2019; Heller et al., 2014; Hussain-Gambles et al., 2004]. Additionally, analysis of race/ethnicity is complicated by a variety of social and environmental factors (e.g., socioeconomic status (SES), access to healthcare, exposure to toxins/pollutants, poor nutrition, etc.) that can confound MRI outcomes if not properly modeled [Crowder and Downey, 2010; Gee and Payne-Sturges, 2004; LeWinn et al., 2017; Nuru-Jeter et al., 2018; Ward et al., 2019]. Unfortunately, stressors that result from systemic racism or discrimination are challenging to measure, but interact with social and environmental health determinants to perpetuate disease inequities in minority populations [Adigbli, 2020].

Fortunately, a number of large-scale initiatives with neuroimaging data have prioritized the recruitment of race and ethnic minorities, including (but not limited to) the Washington HeightsInwood Columbia Aging project (WHICAP; https://www.maelstrom-research.org/mica/individualstudy/whicap), Uncovering Neurodegenerative Insights Through Ethnic Diversity (UNITED; https://www.theunitedconsortium.com) [Adams et al., 2019], the Health and Aging Brain in Latino Elders (HABLE; https://grantome.com/grant/NIH/R01-AG058533-01A1), and the All of Us program (https://allofus.nih.gov/about). These diverse cohorts also exist for studies of brain development (e.g., Adolescent Brain and Cognitive Development (ABCD) Study; https://abcdstudy.org/about/). For researchers with access to these data, we recommend testing 2-way interactions between sex and race/ethnicity on brain outcomes, and 3-way interactions (sex-by-race-by-diagnosis) for clinical research questions. Interactions should be analyzed in conjunction with a rich set of social and environmental measures to account for potential confounders. Testing sex-by-race interactions within and between distinct SES strata may further inform these associations. 


\section{Conclusions}

There are significant sex differences in the mean age of onset of many psychiatric and neurological disorders, in treatment response, symptom severity and disease trajectory, and yet we still lack detailed knowledge of how sex-specific or sex-dependent mechanisms contribute to specific neuroimaging phenotypes. This lack of knowledge - even of basic trajectories of brain disease in males versus females - stalls efforts to identify cures for the most common and debilitating mental health and neurological diseases. As we continue to expand international consortia and improve imaging and medical technology, it is imperative to consider the role of sex as a separate etiological factor in psychiatric and degenerative disease, and ensure that this factor is reflected in our neuroimaging designs. Future work using machine learning will also help to discover sex-dependent interactions with biological, genetic, and environmental factors that influence unique patterns of brain structure and function in males and females across the lifespan.

\section{References}

Adams HHH, Evans TE, Terzikhan N (2019): The Uncovering Neurodegenerative Insights Through Ethnic Diversity consortium. Lancet Neurol 18:915. http://dx.doi.org/10.1016/S1474-4422(19)30324-2.

Adam Y, Meinlschmidt G, Gloster AT, Lieb R (2012): Obsessive-compulsive disorder in the community: 12-month prevalence, comorbidity and impairment. Soc Psychiatry Psychiatr Epidemiol 47:339-349. http://link.springer.com/10.1007/s00127-010-0337-5.

Adigbli G (2020): Race, science and (im)precision medicine. Nat Med 26:1675-1676. http://dx.doi.org/10.1038/s41591-020-1115-x.

Agabio R, Campesi I, Pisanu C, Gessa GL, Franconi F (2016): Sex differences in substance use disorders: focus on side effects. Addict Biol 21:1030-1042. http://dx.doi.org/10.1111/adb.12395.

Ahearn EP, Szymanski BR, Chen P, Sajatovic M, Katz IR, McCarthy JF (2020): Increased Risk of Dementia Among Veterans With Bipolar Disorder or Schizophrenia Receiving Care in the VA Health System. Psychiatr Serv 71:998-1004. http://dx.doi.org/10.1176/appi.ps.201900325.

Akashi T, Takahashi S, Mugikura S, Sato S, Murata T, Umetsu A, Takase K (2017): Ischemic White Matter Lesions Associated With Medullary Arteries: Classification of MRI Findings Based on the Anatomic Arterial Distributions. AJR Am J Roentgenol 209:W160-W168. http://dx.doi.org/10.2214/AJR.16.17231.

Akiki TJ, Averill CL, Wrocklage KM, Schweinsburg B, Scott JC, Martini B, Averill LA, Southwick SM, Krystal JH, Abdallah CG (2017): The Association of PTSD Symptom Severity with Localized Hippocampus and Amygdala Abnormalities. Chronic Stress (Thousand Oaks) 1. http://dx.doi.org/10.1177/2470547017724069.

Almeida OP, Hankey GJ, Yeap BB, Golledge J, Flicker L (2017): Depression as a modifiable factor to decrease the risk of dementia. Transl Psychiatry 7:e1117. http://dx.doi.org/10.1038/tp.2017.90.

Alzheimer's Association (2015): 2015 Alzheimer's disease facts and figures. Alzheimers Dement 11:332- 
384. http://doi.wiley.com/10.1016/j.jalz.2015.02.003.

Ancelin M-L, Carrière I, Artero S, Maller J, Meslin C, Ritchie K, Ryan J, Chaudieu I (2019): Lifetime major depression and grey-matter volume. J Psychiatry Neurosci 44:45-53.

http://dx.doi.org/10.1503/jpn.180026.

2020 Alzheimer's disease facts and figures (2020): . Alzheimers Dement. http://dx.doi.org/10.1002/alz.12068.

Antulov R, Weinstock-Guttman B, Cox JL, Hussein S, Durfee J, Caiola C, Dwyer MG, Bergsland N, Abdelrahman N, Stosic M, Hojnacki D, Munschauer FE, Miletic D, Zivadinov R (2009): Genderrelated differences in MS: a study of conventional and nonconventional MRI measures. Mult Scler 15:345-354. http://dx.doi.org/10.1177/1352458508099479.

Arnold AP, Breedlove SM (1985): Organizational and activational effects of sex steroids on brain and behavior: a reanalysis. Horm Behav 19:469-498. http://dx.doi.org/10.1016/0018-506x(85)90042-x.

Asami T, Yamasue H, Hayano F, Nakamura M, Uehara K, Otsuka T, Roppongi T, Nihashi N, Inoue T, Hirayasu Y (2009): Sexually dimorphic gray matter volume reduction in patients with panic disorder. Psychiatry Res 173:128-134. http://dx.doi.org/10.1016/j.pscychresns.2008.10.004.

Babulal GM, Quiroz YT, Albensi BC, Arenaza-Urquijo E, Astell AJ, Babiloni C, Bahar-Fuchs A, Bell J, Bowman GL, Brickman AM, Chételat G, Ciro C, Cohen AD, Dilworth-Anderson P, Dodge HH, Dreux S, Edland S, Esbensen A, Evered L, Ewers M, Fargo KN, Fortea J, Gonzalez H, Gustafson DR, Head E, Hendrix JA, Hofer SM, Johnson LA, Jutten R, Kilborn K, Lanctôt KL, Manly JJ, Martins RN, Mielke MM, Morris MC, Murray ME, Oh ES, Parra MA, Rissman RA, Roe CM, Santos OA, Scarmeas N, Schneider LS, Schupf N, Sikkes S, Snyder HM, Sohrabi HR, Stern Y, Strydom A, Tang Y, Terrera GM, Teunissen C, Melo van Lent D, Weinborn M, Wesselman L, Wilcock DM, Zetterberg H, O'Bryant SE, International Society to Advance Alzheimer's Research and Treatment, Alzheimer's Association (2019): Perspectives on ethnic and racial disparities in Alzheimer's disease and related dementias: Update and areas of immediate need. Alzheimers Dement 15:292-312. http://dx.doi.org/10.1016/j.jalz.2018.09.009.

Bae S, Sheth C, Legarreta M, McGlade E, Lyoo IK, Yurgelun-Todd DA (2019): Volume and shape analysis of the Hippocampus and amygdala in veterans with traumatic brain injury and posttraumatic stress disorder. Brain Imaging Behav. http://dx.doi.org/10.1007/s11682-019-00127-2.

Ballmaier M, O'Brien JT, Burton EJ, Thompson PM, Rex DE, Narr KL, McKeith IG, DeLuca H, Toga AW (2004): Comparing gray matter loss profiles between dementia with Lewy bodies and Alzheimer's disease using cortical pattern matching: diagnosis and gender effects. Neuroimage 23:325-335. http://dx.doi.org/10.1016/j.neuroimage.2004.04.026.

Barker-Collo S, Bennett DA, Krishnamurthi RV, Parmar P, Feigin VL, Naghavi M, Forouzanfar MH, Johnson CO, Nguyen G, Mensah GA, Vos T, Murray CJL, Roth GA, GBD 2013 Writing Group, GBD 2013 Stroke Panel Experts Group (2015): Sex Differences in Stroke Incidence, Prevalence, Mortality and Disability-Adjusted Life Years: Results from the Global Burden of Disease Study 2013. Neuroepidemiology 45:203-214. http://dx.doi.org/10.1159/000441103.

Barth C, Villringer A, Sacher J (2015): Sex hormones affect neurotransmitters and shape the adult female brain during hormonal transition periods. Front Neurosci 9:37. http://dx.doi.org/10.3389/fnins.2015.00037.

Basser PJ, Pierpaoli C (1996): Microstructural and physiological features of tissues elucidated by quantitative-diffusion-tensor MRI. J Magn Reson B 111:209-219. http://dx.doi.org/10.1006/jmrb.1996.0086.

Becker JB, Hu M (2008): Sex differences in drug abuse. Front Neuroendocrinol 29:36-47. http://dx.doi.org/10.1016/j.yfrne.2007.07.003.

Beery AK, Zucker I (2011): Sex bias in neuroscience and biomedical research. Neurosci Biobehav Rev 35:565-572. http://dx.doi.org/10.1016/j.neubiorev.2010.07.002.

Bekker MHJ, van Mens-Verhulst J (2007): Anxiety disorders: sex differences in prevalence, degree, and background, but gender-neutral treatment. Gend Med 4 Suppl B:S178-93. http://dx.doi.org/10.1016/s1550-8579(07)80057-x.

Bell CN, Thorpe RJ Jr, Bowie JV, LaVeist TA (2018): Race disparities in cardiovascular disease risk factors within socioeconomic status strata. Ann Epidemiol 28:147-152. http://dx.doi.org/10.1016/j.annepidem.2017.12.007.

Benatti B, Celebre L, Girone N, Priori A, Bruno A, Viganò C, Hollander E, Dell'Osso B (2020): Clinical characteristics and comorbidity associated with female gender in obsessive-compulsive disorder. $\mathrm{J}$ 
Psychiatr Res 131:209-214. http://dx.doi.org/10.1016/j.jpsychires.2020.09.019.

Benazzouz A, Mamad O, Abedi P, Bouali-Benazzouz R, Chetrit J (2014): Involvement of dopamine loss in extrastriatal basal ganglia nuclei in the pathophysiology of Parkinson's disease. Front Aging Neurosci 6:87. http://dx.doi.org/10.3389/fnagi.2014.00087.

Benjamin EJ, Blaha MJ, Chiuve SE, Cushman M, Das SR, Deo R, de Ferranti SD, Floyd J, Fornage M, Gillespie C, Isasi CR, Jiménez MC, Jordan LC, Judd SE, Lackland D, Lichtman JH, Lisabeth L, Liu S, Longenecker CT, Mackey RH, Matsushita K, Mozaffarian D, Mussolino ME, Nasir K, Neumar RW, Palaniappan L, Pandey DK, Thiagarajan RR, Reeves MJ, Ritchey M, Rodriguez CJ, Roth GA, Rosamond WD, Sasson C, Towfighi A, Tsao CW, Turner MB, Virani SS, Voeks JH, Willey JZ, Wilkins JT, Wu JH, Alger HM, Wong SS, Muntner P, American Heart Association Statistics Committee and Stroke Statistics Subcommittee (2017): Heart Disease and Stroke Statistics-2017 Update: A Report From the American Heart Association. Circulation 135:e146-e603. http://dx.doi.org/10.1161/CIR.0000000000000485.

Bennett IJ, Madden DJ, Vaidya CJ, Howard DV, Howard JH Jr (2010): Age-related differences in multiple measures of white matter integrity: A diffusion tensor imaging study of healthy aging. Hum Brain Mapp 31:378-390. http://dx.doi.org/10.1002/hbm.20872.

Benowitz NL, Lessov-Schlaggar CN, Swan GE, Jacob P 3rd (2006): Female sex and oral contraceptive use accelerate nicotine metabolism. Clin Pharmacol Ther 79:480-488. http://dx.doi.org/10.1016/j.clpt.2006.01.008.

Berman SB, Miller-Patterson C (2019): PD and DLB: Brain imaging in Parkinson's disease and dementia with Lewy bodies. Progress in Molecular Biology and Translational Science. http://dx.doi.org/10.1016/bs.pmbts.2019.07.009.

Bidlingmaier F, Wagner-Barnack M, Butenandt O, Knorr D (1973): Plasma estrogens in childhood and puberty under physiologic and pathologic conditions. Pediatr Res 7:901-907. http://dx.doi.org/10.1203/00006450-197311000-00006.

Biggio JR Jr (2020): Research in Pregnant Subjects: Increasingly Important, but Challenging. Ochsner J 20:39-43. http://dx.doi.org/10.31486/toj.19.0077.

Bobzean SAM, DeNobrega AK, Perrotti LI (2014): Sex differences in the neurobiology of drug addiction. Exp Neurol 259:64-74. http://dx.doi.org/10.1016/j.expneurol.2014.01.022.

Boedhoe PSW, Schmaal L, Abe Y, Alonso P, Ameis SH, Anticevic A, Arnold PD, Batistuzzo MC, Benedetti F, Beucke JC, Bollettini I, Bose A, Brem S, Calvo A, Calvo R, Cheng Y, Cho KIK, Ciullo V, Dallaspezia S, Denys D, Feusner JD, Fitzgerald KD, Fouche J-P, Fridgeirsson EA, Gruner P, Hanna GL, Hibar DP, Hoexter MQ, Hu H, Huyser C, Jahanshad N, James A, Kathmann N, Kaufmann C, Koch K, Kwon JS, Lazaro L, Lochner C, Marsh R, Martínez-Zalacaín I, Mataix-Cols D, Menchón JM, Minuzzi L, Morer A, Nakamae T, Nakao T, Narayanaswamy JC, Nishida S, Nurmi E, O’Neill J, Piacentini J, Piras F, Piras F, Reddy YCJ, Reess TJ, Sakai Y, Sato JR, Simpson HB, Soreni N, Soriano-Mas C, Spalletta G, Stevens MC, Szeszko PR, Tolin DF, van Wingen GA, Venkatasubramanian G, Walitza S, Wang Z, Yun J-Y, ENIGMA-OCD Working Group, Thompson PM, Stein DJ, van den Heuvel OA, ENIGMA OCD Working Group (2018): Cortical Abnormalities Associated With Pediatric and Adult Obsessive-Compulsive Disorder: Findings From the ENIGMA Obsessive-Compulsive Disorder Working Group. Am J Psychiatry 175:453-462. http://dx.doi.org/10.1176/appi.ajp.2017.17050485.

Boedhoe PSW, Schmaal L, Abe Y, Ameis SH, Arnold PD, Batistuzzo MC, Benedetti F, Beucke JC, Bollettini I, Bose A, Brem S, Calvo A, Cheng Y, Cho KIK, Dallaspezia S, Denys D, Fitzgerald KD, Fouche J-P, Giménez M, Gruner P, Hanna GL, Hibar DP, Hoexter MQ, Hu H, Huyser C, Ikari K, Jahanshad N, Kathmann N, Kaufmann C, Koch K, Kwon JS, Lazaro L, Liu Y, Lochner C, Marsh R, Martínez-Zalacaín I, Mataix-Cols D, Menchón JM, Minuzzi L, Nakamae T, Nakao T, Narayanaswamy JC, Piras F, Piras F, Pittenger C, Reddy YCJ, Sato JR, Simpson HB, Soreni N, Soriano-Mas C, Spalletta G, Stevens MC, Szeszko PR, Tolin DF, Venkatasubramanian G, Walitza S, Wang Z, van Wingen GA, Xu J, Xu X, Yun J-Y, Zhao Q, ENIGMA OCD Working Group, Thompson PM, Stein DJ, van den Heuvel OA (2017): Distinct Subcortical Volume Alterations in Pediatric and Adult OCD: A Worldwide Meta- and Mega-Analysis. Am J Psychiatry 174:60-69. http://dx.doi.org/10.1176/appi.ajp.2016.16020201.

Bondi MW, Edmonds EC, Salmon DP (2017): Alzheimer's Disease: Past, Present, and Future. J Int Neuropsychol Soc 23:818-831. http://dx.doi.org/10.1017/S135561771700100X.

Braak H, Braak E (1991): Neuropathological stageing of Alzheimer-related changes. Acta Neuropathol 
82:239-259. http://dx.doi.org/10.1007/bf00308809.

Braak H, Del Tredici K (2015): The preclinical phase of the pathological process underlying sporadic Alzheimer's disease. Brain 138:2814-2833. http://dx.doi.org/10.1093/brain/awv236.

Braak H, Thal DR, Ghebremedhin E, Del Tredici K (2011): Stages of the pathologic process in Alzheimer disease: age categories from 1 to 100 years. J Neuropathol Exp Neurol 70:960-969. http://dx.doi.org/10.1097/NEN.0b013e318232a379.

Brakoulias V, Starcevic V, Belloch A, Brown C, Ferrao YA, Fontenelle LF, Lochner C, Marazziti D, Matsunaga H, Miguel EC, Reddy YCJ, do Rosario MC, Shavitt RG, Shyam Sundar A, Stein DJ, Torres AR, Viswasam K (2017): Comorbidity, age of onset and suicidality in obsessive-compulsive disorder (OCD): An international collaboration. Compr Psychiatry 76:79-86. http://www.sciencedirect.com/science/article/pii/S0010440X1630699X.

Brookmeyer R, Abdalla N, Kawas CH, Corrada MM (2018): Forecasting the prevalence of preclinical and clinical Alzheimer's disease in the United States. Alzheimers Dement 14:121-129. http://dx.doi.org/10.1016/j.jalz.2017.10.009.

Brun CC, Leporé N, Luders E, Chou Y-Y, Madsen SK, Toga AW, Thompson PM (2009): Sex differences in brain structure in auditory and cingulate regions. Neuroreport 20:930-935. http://dx.doi.org/10.1097/wnr.0b013e32832c5e65.

Bruun M, Koikkalainen J, Rhodius-Meester HFM, Baroni M, Gjerum L, van Gils M, Soininen H, Remes AM, Hartikainen P, Waldemar G, Mecocci P, Barkhof F, Pijnenburg Y, van der Flier WM, Hasselbalch SG, Lötjönen J, Frederiksen KS (2019): Detecting frontotemporal dementia syndromes using MRI biomarkers. Neuroimage Clin 22:101711. http://dx.doi.org/10.1016/j.nicl.2019.101711.

Bryant NL, Buchanan RW, Vladar K, Breier A, Rothman M (1999): Gender Differences in Temporal Lobe Structures of Patients With Schizophrenia: A Volumetric MRI Study. AJP 156:603-609. https://ajp.psychiatryonline.org/doi/abs/10.1176/ajp.156.4.603.

Buckley RF, Waller M, Masters CL, Dobson A (2019): To What Extent Does Age at Death Account for Sex Differences in Rates of Mortality From Alzheimer Disease? Am J Epidemiol 188:1213-1223. http://dx.doi.org/10.1093/aje/kwz048.

Bullmore E, Sporns O (2009): Complex brain networks: graph theoretical analysis of structural and functional systems. Nat Rev Neurosci 10:186-198. http://dx.doi.org/10.1038/nrn2575.

Burciu RG, Ofori E, Archer DB, Wu SS, Pasternak O, McFarland NR, Okun MS, Vaillancourt DE (2017): Progression marker of Parkinson's disease: a 4-year multi-site imaging study. Brain 140:2183-2192. http://dx.doi.org/10.1093/brain/awx146.

Burke SL, Hu T, Fava NM, Li T, Rodriguez MJ, Schuldiner KL, Burgess A, Laird A (2019): Sex differences in the development of mild cognitive impairment and probable Alzheimer's disease as predicted by hippocampal volume or white matter hyperintensities. Journal of Women \& Aging. http://dx.doi.org/10.1080/08952841.2018.1419476.

Butler ER, Chen A, Ramadan R, Ruparel K, Moore TM, Zhang F, Shou H, Gur RC, Nichols TE, Shinohara RT (2020): Statistical Pitfalls in Brain Age Analyses. bioRxiv. http://dx.doi.org/10.1101/2020.06.21.163741.

Button KS, loannidis JPA, Mokrysz C, Nosek BA, Flint J, Robinson ESJ, Munafò MR (2013): Power failure: why small sample size undermines the reliability of neuroscience. Nat Rev Neurosci 14:365376. http://dx.doi.org/10.1038/nrn3475.

Calabrese M, Battaglini M, Giorgio A, Atzori M, Bernardi V, Mattisi I, Gallo P, De Stefano N (2010): Imaging distribution and frequency of cortical lesions in patients with multiple sclerosis. Neurology 75:1234-1240. http://dx.doi.org/10.1212/WNL.0b013e3181f5d4da.

Caldwell JZK, Berg J-L, Shan G, Cummings JL, Banks SJ, Alzheimer's Disease Neuroimaging Initiative (2018): Sex Moderates the Impact of Diagnosis and Amyloid PET Positivity on Hippocampal Subfield Volume. J Alzheimers Dis 64:79-89. http://dx.doi.org/10.3233/JAD-180028.

Campbell BC, Dreber A, Apicella CL, Eisenberg DTA, Gray PB, Little AC, Garcia JR, Zamore RS, Lum JK (2010): Testosterone exposure, dopaminergic reward, and sensation-seeking in young men. Physiol Behav 99:451-456. http://dx.doi.org/10.1016/j.physbeh.2009.12.011.

Canevelli M, Bruno G, Grande G, Quarata F, Raganato R, Remiddi F, Valletta M, Zaccaria V, Vanacore N, Cesari M (2019): Race reporting and disparities in clinical trials on Alzheimer's disease: A systematic review. Neurosci Biobehav Rev 101:122-128. http://dx.doi.org/10.1016/j.neubiorev.2019.03.020.

Castle DJ, Deale A, Marks IM (1995): Gender differences in obsessive compulsive disorder. Aust N Z J 
Psychiatry 29:114-117. http://dx.doi.org/10.3109/00048679509075899.

Casto KV, Edwards DA (2016): Testosterone, cortisol, and human competition. Horm Behav 82:21-37. http://dx.doi.org/10.1016/j.yhbeh.2016.04.004.

Catenaccio E, Mu W, Lipton ML (2016): Estrogen- and progesterone-mediated structural neuroplasticity in women: evidence from neuroimaging. Brain Structure and Function. http://dx.doi.org/10.1007/s00429-016-1197-x.

Cath DC, Nizar K, Boomsma D, Mathews CA (2017): Age-Specific Prevalence of Hoarding and Obsessive Compulsive Disorder: A Population-Based Study. Am J Geriatr Psychiatry 25:245-255. http://dx.doi.org/10.1016/j.jagp.2016.11.006.

Cavanagh A, Wilson CJ, Kavanagh DJ, Caputi P (2017): Differences in the Expression of Symptoms in Men Versus Women with Depression: A Systematic Review and Meta-analysis. Harv Rev Psychiatry 25:29-38. http://dx.doi.org/10.1097/HRP.0000000000000128.

Cerri S, Mus L, Blandini F (2019): Parkinson's Disease in Women and Men: What's the Difference? J Parkinsons Dis 9:501-515. http://dx.doi.org/10.3233/JPD-191683.

Chadwick AJ, Baruah R (2020): Gender disparity and implicit gender bias amongst doctors in intensive care medicine: A "disease"we need to recognise and treat. Pediatr Crit Care Med 21:12-17. https://journals.sagepub.com/doi/abs/10.1177/1751143719870469.

Chapman EN, Kaatz A, Carnes M (2013): Physicians and implicit bias: how doctors may unwittingly perpetuate health care disparities. J Gen Intern Med 28:1504-1510. http://dx.doi.org/10.1007/s11606-013-2441-1.

Chare L, Hodges JR, Leyton CE, McGinley C, Tan RH, Kril JJ, Halliday GM (2014): New criteria for frontotemporal dementia syndromes: clinical and pathological diagnostic implications. J Neurol Neurosurg Psychiatry 85:865-870. http://dx.doi.org/10.1136/jnnp-2013-306948.

Cheng H-C, Ulane CM, Burke RE (2010): Clinical progression in Parkinson disease and the neurobiology of axons. Ann Neurol 67:715-725. http://dx.doi.org/10.1002/ana.21995.

Ching CRK, Abaryan Z, Santhalingam V, Zhu AH, Bright JK, Jahanshad N, Thompson PM (2020): Sexdependent age trajectories of subcortical brain structures: analysis of large-scale percentile models and shape morphometry. In: . 16th International Symposium on Medical Information Processing and Analysis. International Society for Optics and Photonics. Vol. 11583, p 1158306.

https://www.spiedigitallibrary.org/conference-proceedings-of-spie/11583/1158306/Sex-dependentage-trajectories-of-subcortical-brain-structures--

analysis/10.1117/12.2579556.short?casa_token=3Ep6g2OeZBoAAAAA:GK1MM947c4_K5IdDNdHj0CT7yJfcKtBZ19-ry_LAjb8VM77InMKQ80DgtwcvqoAG0910dUJ5fk.

Chiu P-Y, Teng P-R, Wei C-Y, Wang C-W, Tsai C-T (2018): Gender difference in the association and presentation of visual hallucinations in dementia with Lewy bodies: a cross-sectional study. Int $J$ Geriatr Psychiatry 33:193-199. http://dx.doi.org/10.1002/gps.4706.

Cholerton B, Johnson CO, Fish B, Quinn JF, Chung KA, Peterson-Hiller AL, Rosenthal LS, Dawson TM, Albert MS, Hu S-C, Mata IF, Leverenz JB, Poston KL, Montine TJ, Zabetian CP, Edwards KL (2018): Sex differences in progression to mild cognitive impairment and dementia in Parkinson's disease. Parkinsonism Relat Disord 50:29-36. http://www.sciencedirect.com/science/article/pii/S1353802018300439.

Clayton JA (2018): Applying the new SABV (sex as a biological variable) policy to research and clinical care. Physiol Behav 187:2-5. http://dx.doi.org/10.1016/j.physbeh.2017.08.012.

Comasco E, Frokjaer VG, Sundström-Poromaa I (2014): Functional and molecular neuroimaging of menopause and hormone replacement therapy. Front Neurosci 8:388. http://dx.doi.org/10.3389/fnins.2014.00388.

Cortese R, Collorone S, Ciccarelli O, Toosy AT (2019): Advances in brain imaging in multiple sclerosis. Ther Adv Neurol Disord 12:1756286419859722. http://dx.doi.org/10.1177/1756286419859722.

Cosgrove KP, Mazure CM, Staley JK (2007): Evolving knowledge of sex differences in brain structure, function, and chemistry. Biol Psychiatry 62:847-855. http://dx.doi.org/10.1016/j.biopsych.2007.03.001.

Costantine MM, Landon MB, Saade GR (2020): Protection by Exclusion: Another Missed Opportunity to Include Pregnant Women in Research During the Coronavirus Disease 2019 (COVID-19) Pandemic. Obstet Gynecol 136:26-28. http://dx.doi.org/10.1097/AOG.0000000000003924.

Cousins KAQ, Grossman M (2017): Evidence of semantic processing impairments in behavioural variant frontotemporal dementia and Parkinson's disease. Curr Opin Neurol 30:617-622. 
http://dx.doi.org/10.1097/WCO.0000000000000498.

Crowder K, Downey L (2010): Interneighborhood migration, race, and environmental hazards: modeling microlevel processes of environmental inequality. AJS 115:1110-1149. http://dx.doi.org/10.1086/649576.

Daniels K, Abma JC (2020): Current contraceptive status among women aged 15--49: United States, 2017--2019. https://stacks.cdc.gov/view/cdc/95692.

Daugherty SL, Blair IV, Havranek EP, Furniss A, Dickinson LM, Karimkhani E, Main DS, Masoudi FA (2017): Implicit Gender Bias and the Use of Cardiovascular Tests Among Cardiologists. J Am Heart Assoc 6. http://dx.doi.org/10.1161/JAHA.117.006872.

Davey RA, Grossmann M (2016): Androgen Receptor Structure, Function and Biology: From Bench to Bedside. Clin Biochem Rev 37:3-15. https://www.ncbi.nlm.nih.gov/pubmed/27057074.

Deak T, Quinn M, Cidlowski JA, Victoria NC, Murphy AZ, Sheridan JF (2015): Neuroimmune mechanisms of stress: sex differences, developmental plasticity, and implications for pharmacotherapy of stressrelated disease. Stress 18:367-380. http://dx.doi.org/10.3109/10253890.2015.1053451.

Del-Ben CM, Graeff FG (2009): Panic disorder: is the PAG involved? Neural Plast 2009:108135. http://dx.doi.org/10.1155/2009/108135.

Del Casale A, Serata D, Rapinesi C, Kotzalidis GD, Angeletti G, Tatarelli R, Ferracuti S, Girardi P (2013): Structural neuroimaging in patients with panic disorder: findings and limitations of recent studies. Psychiatr Danub 25:108-114. https://www.ncbi.nlm.nih.gov/pubmed/23793273.

De Leeuw FE, de Groot JC, Achten E, Oudkerk M, Ramos LMP, Heijboer R, Hofman A, Jolles J, Van Gijn J, Breteler MMB (2001): Prevalence of cerebral white matter lesions in elderly people: a population based magnetic resonance imaging study. The Rotterdam Scan Study. J Neurol Neurosurg Psychiatry 70:9-14. https://jnnp.bmj.com/content/70/1/9.short.

Del Río JP, Alliende MI, Molina N, Serrano FG, Molina S, Vigil P (2018): Steroid Hormones and Their Action in Women's Brains: The Importance of Hormonal Balance. Front Public Health 6:141. http://dx.doi.org/10.3389/fpubh.2018.00141.

Dennis EL, Disner SG, Fani N, Salminen LE, Logue M, Clarke EK, Haswell CC, Averill CL, Baugh LA, Bomyea J, Bruce SE, Cha J, Choi K, Davenport ND, Densmore M, du Plessis S, Forster GL, Frijling JL, Gonenc A, Gruber S, Grupe DW, Guenette JP, Hayes J, Hofmann D, Ipser J, Jovanovic T, Kelly S, Kennis M, Kinzel P, Koch SBJ, Koerte I, Koopowitz S, Korgaonkar M, Krystal J, Lebois LAM, Li G, Magnotta VA, Manthey A, May GJ, Menefee DS, Nawijn L, Nelson SM, Neufeld RWJ, Nitschke JB, O'Doherty D, Peverill M, Ressler KJ, Roos A, Sheridan MA, Sierk A, Simmons A, Simons RM, Simons JS, Stevens J, Suarez-Jimenez B, Sullivan DR, Théberge J, Tran JK, van den Heuvel L, van der Werff SJA, van Rooij SJH, van Zuiden M, Velez C, Verfaellie M, Vermeiren RRJM, Wade BSC, Wager T, Walter H, Winternitz S, Wolff J, York G, Zhu Y, Zhu X, Abdallah CG, Bryant R, Daniels JK, Davidson RJ, Fercho KA, Franz C, Geuze E, Gordon EM, Kaufman ML, Kremen WS, Lagopoulos J, Lanius RA, Lyons MJ, McCauley SR, McGlinchey R, McLaughlin KA, Milberg W, Neria Y, Olff M, Seedat S, Shenton M, Sponheim SR, Stein DJ, Stein MB, Straube T, Tate DF, van der Wee NJA, Veltman DJ, Wang L, Wilde EA, Thompson PM, Kochunov P, Jahanshad N, Morey RA (2019): Altered white matter microstructural organization in posttraumatic stress disorder across 3047 adults: results from the PGC-ENIGMA PTSD consortium. Mol Psychiatry. http://dx.doi.org/10.1038/s41380019-0631-x.

Dennis EL, Rashid F, Faskowitz J, Jin Y, McMahon KL, de Zubicaray GI, Martin NG, Hickie IB, Wright MJ, Jahanshad N, Thompson PM (2017): Mapping age effects along fiber tracts in young adults. Proc IEEE Int Symp Biomed Imaging 2017:101-104. http://dx.doi.org/10.1109/ISBI.2017.7950478.

Dhamoon MS, McClure LA, White CL, Lakshminarayan K, Benavente OR, Elkind MSV, SPS3 Investigators (2015): Long-term disability after lacunar stroke: secondary prevention of small subcortical strokes. Neurology 84:1002-1008. http://dx.doi.org/10.1212/WNL.0000000000001331.

Dima D, Papachristou E, Modabbernia A, Doucet GE (2020): Subcortical Volume Trajectories across the Lifespan: Data from 18,605 healthy individuals aged 3-90 years. bioRxiv. https://www.biorxiv.org/content/10.1101/2020.05.05.079475v1.abstract.

Diniz BS, Teixeira AL, Cao F, Gildengers A, Soares JC, Butters MA, Reynolds CF 3rd (2017): History of Bipolar Disorder and the Risk of Dementia: A Systematic Review and Meta-Analysis. Am J Geriatr Psychiatry 25:357-362. http://dx.doi.org/10.1016/j.jagp.2016.11.014.

Duggal HS, Muddasani S, Keshavan MS (2005): Insular volumes in first-episode schizophrenia: gender effect. Schizophr Res 73:113-120. http://dx.doi.org/10.1016/j.schres.2004.08.027. 
Dula AN, Mlynash M, Zuck ND, Albers GW, Warach SJ, DEFUSE 3 Investigators (2020): Neuroimaging in Ischemic Stroke Is Different Between Men and Women in the DEFUSE 3 Cohort. Stroke 51:481-488. http://dx.doi.org/10.1161/STROKEAHA.119.028205.

Dunlop K, Talishinsky A, Liston C (2019): Intrinsic Brain Network Biomarkers of Antidepressant Response: a Review. Curr Psychiatry Rep 21:87. http://dx.doi.org/10.1007/s11920-019-1072-6.

Earls F (1987): Sex differences in psychiatric disorders: origins and developmental influences. Psychiatr Dev 5:1-23. https://www.ncbi.nlm.nih.gov/pubmed/3601929.

Eberling JL, Wu C, Haan MN, Mungas D (2003): Preliminary evidence that estrogen protects against agerelated hippocampal atrophy. Neurobiology of. https://www.sciencedirect.com/science/article/pii/S0197458002000568.

van Eersel G, Bouwmeester S, Verkoeijen P, Polak MG (2017): The misuse of ANCOVA in neuroimaging studies. psyarxiv.com/qcsbz.

van Eijk L, Hansell NK, Strike LT, Couvy-Duchesne B, de Zubicaray GI, Thompson PM, McMahon KL, Zietsch BP, Wright MJ (2020): Region-Specific Sex Differences in the Hippocampus. Neuroimage:116781. http://dx.doi.org/10.1016/j.neuroimage.2020.116781.

Eisenegger C, Haushofer J, Fehr E (2011): The role of testosterone in social interaction. Trends Cogn Sci 15:263-271. http://dx.doi.org/10.1016/j.tics.2011.04.008.

ENIGMA (04/2017): ENIGMA Structural Imaging Protocols. University of Southern California. http://enigma.ini.usc.edu/.

van Erp TGM, Walton E, Hibar DP, Schmaal L, Jiang W, Glahn DC, Pearlson GD, Yao N, Fukunaga M, Hashimoto R, Okada N, Yamamori H, Bustillo JR, Clark VP, Agartz I, Mueller BA, Cahn W, de Zwarte SMC, Hulshoff Pol HE, Kahn RS, Ophoff RA, van Haren NEM, Andreassen OA, Dale AM, Doan NT, Gurholt TP, Hartberg CB, Haukvik UK, Jørgensen KN, Lagerberg TV, Melle I, Westlye LT, Gruber O, Kraemer B, Richter A, Zilles D, Calhoun VD, Crespo-Facorro B, Roiz-Santiañez R, TordesillasGutiérrez D, Loughland C, Carr VJ, Catts S, Cropley VL, Fullerton JM, Green MJ, Henskens FA, Jablensky A, Lenroot RK, Mowry BJ, Michie PT, Pantelis C, Quidé Y, Schall U, Scott RJ, Cairns MJ, Seal M, Tooney PA, Rasser PE, Cooper G, Shannon Weickert C, Weickert TW, Morris DW, Hong E, Kochunov P, Beard LM, Gur RE, Gur RC, Satterthwaite TD, Wolf DH, Belger A, Brown GG, Ford JM, Macciardi F, Mathalon DH, O'Leary DS, Potkin SG, Preda A, Voyvodic J, Lim KO, McEwen S, Yang F, Tan Y, Tan S, Wang Z, Fan F, Chen J, Xiang H, Tang S, Guo H, Wan P, Wei D, Bockholt HJ, Ehrlich S, Wolthusen RPF, King MD, Shoemaker JM, Sponheim SR, De Haan L, Koenders L, Machielsen MW, van Amelsvoort T, Veltman DJ, Assogna F, Banaj N, de Rossi P, Iorio M, Piras F, Spalletta G, McKenna PJ, Pomarol-Clotet E, Salvador R, Corvin A, Donohoe G, Kelly S, Whelan CD, Dickie EW, Rotenberg D, Voineskos AN, Ciufolini S, Radua J, Dazzan P, Murray R, Reis Marques T, Simmons A, Borgwardt S, Egloff L, Harrisberger F, Riecher-Rössler A, Smieskova R, Alpert KI, Wang L, Jönsson EG, Koops S, Sommer IEC, Bertolino A, Bonvino A, Di Giorgio A, Neilson E, Mayer AR, Stephen JM, Kwon JS, Yun J-Y, Cannon DM, McDonald C, Lebedeva I, Tomyshev AS, Akhadov T, Kaleda V, Fatouros-Bergman H, Flyckt L, Karolinska Schizophrenia Project, Busatto GF, Rosa PGP, Serpa MH, Zanetti MV, Hoschl C, Skoch A, Spaniel F, Tomecek D, Hagenaars SP, Mclntosh AM, Whalley HC, Lawrie SM, Knöchel C, Oertel-Knöchel V, Stäblein M, Howells FM, Stein DJ, Temmingh HS, Uhlmann A, Lopez-Jaramillo C, Dima D, McMahon A, Faskowitz JI, Gutman BA, Jahanshad N, Thompson PM, Turner JA (2018): Cortical Brain Abnormalities in 4474 Individuals With Schizophrenia and 5098 Control Subjects via the Enhancing Neuro Imaging Genetics Through Meta Analysis (ENIGMA) Consortium. Biol Psychiatry 84:644-654. http://dx.doi.org/10.1016/j.biopsych.2018.04.023.

Eshaghi A, Young A, Wijertane P, Prados F, Arnold DL, Narayanan S, Guttmann CRG, Barkhof F, Alexander DC, Thompson AJ, Chard D, Ciccarelli O (2019): Defining multiple sclerosis subtypes using machine learning. medRxiv. medRxiv. http://medrxiv.org/lookup/doi/10.1101/19011080.

Eversfield CL, Orton LD (2019): Auditory and visual hallucination prevalence in Parkinson's disease and dementia with Lewy bodies: a systematic review and meta-analysis. Psychol Med 49:2342-2353. http://dx.doi.org/10.1017/S0033291718003161.

Exner C, Nehrkorn B, Martin V, Huber M, Shiratori K, Rief W (2008): Sex-dependent hippocampal volume reductions in schizophrenia relate to episodic memory deficits. J Neuropsychiatry Clin Neurosci 20:227-230. http://dx.doi.org/10.1176/jnp.2008.20.2.227.

Fahn JKS, Lang Pablo A, Poewe ALW, Sampaio SLC, Le Witt Glenn T. Stebbins PA, Dodel ORR, Dubois ASB, Teresi Robert J, van Hilten Joseph Jankovic JJ, La Pelle N (2008): The MDS-sponsored 
Revision of the Unified Parkinson's Disease Rating Scale. Movement Disorders Society. https://www.movementdisorders.org/MDS-Files1/PDFs/Rating-Scales/MDSUPDRS_English_FINAL_Updated_August2019.pdf.

Fan L, Li H, Zhuo J, Zhang Y, Wang J, Chen L, Yang Z, Chu C, Xie S, Laird AR, Fox PT, Eickhoff SB, Yu C, Jiang T (2016): The Human Brainnetome Atlas: A New Brain Atlas Based on Connectional Architecture. Cereb Cortex 26:3508-3526. http://dx.doi.org/10.1093/cercor/bhw157.

Farokhian F, Yang C, Beheshti I, Matsuda H, Wu S (2017): Age-Related Gray and White Matter Changes in Normal Adult Brains. Aging Dis 8:899-909. http://dx.doi.org/10.14336/AD.2017.0502.

Farrer LA, Adrienne Cupples L, Haines JL, Hyman B, Kukull WA, Mayeux R, Myers RH, Pericak-Vance MA, Risch N, van Duijn CM (1997): Effects of Age, Sex, and Ethnicity on the Association Between Apolipoprotein E Genotype and Alzheimer Disease: A Meta-analysis. JAMA 278:1349-1356. https://jamanetwork.com/journals/jama/article-abstract/418446?casa_token=i3XNHa9B9EAAAAA:LXqt0wNHXb1VXYMfR4DMtOzfp9Sccd3qr-IAtATOH5BMR0BHMy957Y_OvVaf4xaD7poYIKvZeA.

Fattore $\bar{L}$, Melis M, Fadda P, Fratta W (2014): Sex differences in addictive disorders. Front Neuroendocrinol 35:272-284. http://dx.doi.org/10.1016/j.yfrne.2014.04.003.

Fawcett EJ, Power H, Fawcett JM (2020): Women Are at Greater Risk of OCD Than Men: A MetaAnalytic Review of OCD Prevalence Worldwide. J Clin Psychiatry 81. http://dx.doi.org/10.4088/JCP.19r13085.

Feldman HA, Longcope C, Derby CA, Johannes CB, Araujo AB, Coviello AD, Bremner WJ, McKinlay JB (2002): Age trends in the level of serum testosterone and other hormones in middle-aged men: longitudinal results from the Massachusetts male aging study. J Clin Endocrinol Metab 87:589-598. http://dx.doi.org/10.1210/jcem.87.2.8201.

Ferrari AJ, Stockings E, Khoo J-P, Erskine HE, Degenhardt L, Vos T, Whiteford HA (2016): The prevalence and burden of bipolar disorder: findings from the Global Burden of Disease Study 2013. Bipolar Disord 18:440-450. http://dx.doi.org/10.1111/bdi.12423.

Ferretti MT, Iulita MF, Cavedo E, Chiesa PA, Schumacher Dimech A, Santuccione Chadha A, Baracchi F, Girouard H, Misoch S, Giacobini E, Depypere H, Hampel H, Women's Brain Project and the Alzheimer Precision Medicine Initiative (2018): Sex differences in Alzheimer disease - the gateway to precision medicine. Nat Rev Neurol 14:457-469. http://dx.doi.org/10.1038/s41582-018-0032-9.

Filippi M, Preziosa P, Banwell BL, Barkhof F, Ciccarelli O, De Stefano N, Geurts JJG, Paul F, Reich DS, Toosy AT, Traboulsee A, Wattjes MP, Yousry TA, Gass A, Lubetzki C, Weinshenker BG, Rocca MA (2019): Assessment of lesions on magnetic resonance imaging in multiple sclerosis: practical guidelines. Brain 142:1858-1875. https://academic.oup.com/brain/article/142/7/1858/5519813.

Filon JR, Intorcia AJ, Sue LI, Vazquez Arreola E, Wilson J, Davis KJ, Sabbagh MN, Belden CM, Caselli RJ, Adler CH, Woodruff BK, Rapscak SZ, Ahern GL, Burke AD, Jacobson S, Shill HA, DriverDunckley E, Chen K, Reiman EM, Beach TG, Serrano GE (2016): Gender Differences in Alzheimer Disease: Brain Atrophy, Histopathology Burden, and Cognition. J Neuropathol Exp Neurol. http://dx.doi.org/10.1093/jnen/nlw047.

Fischl B (2012): FreeSurfer. Neuroimage 62:774-781. http://dx.doi.org/10.1016/j.neuroimage.2012.01.021.

Forest MG, Sizonenko PC, Cathiard AM, Bertrand J (1974): Hypophyso-Gonadal Function in Humans during the First Year of Life: I. EVIDENCE FOR TESTICULAR ACTIVITY IN EARLY INFANCY. J Clin Invest 53:819-828. https://doi.org/10.1172/JCl107621.

Fouche J-P, du Plessis S, Hattingh C, Roos A, Lochner C, Soriano-Mas C, Sato JR, Nakamae T, Nishida S, Kwon JS, Jung WH, Mataix-Cols D, Hoexter MQ, Alonso P, OCD Brain Imaging Consortium, de Wit SJ, Veltman DJ, Stein DJ, van den Heuvel OA (2017): Cortical thickness in obsessivecompulsive disorder: multisite mega-analysis of 780 brain scans from six centres. Br J Psychiatry 210:67-74. http://dx.doi.org/10.1192/bjp.bp.115.164020.

Franconi F, Campesi I (2014): Sex and gender influences on pharmacological response: an overview. Expert Rev Clin Pharmacol 7:469-485. http://dx.doi.org/10.1586/17512433.2014.922866.

Franconi F, Sanna M, Straface E, Chessa R, Rosano G (2012): Pharmacokinetics and Pharmacodynamics: The Role of Sex and Gender. In: Oertelt-Prigione, S, Regitz-Zagrosek, V, editors. Sex and Gender Aspects in Clinical Medicine. London: Springer London. pp 183-194. https://doi.org/10.1007/978-0-85729-832-4_12.

Frangou S, Modabbernia A, Doucet GE (2020a): Cortical Thickness Trajectories across the Lifespan: 
Data from 17,075 healthy individuals aged 3-90 years. bioRxiv. https://www.biorxiv.org/content/10.1101/2020.05.05.077834v1.abstract.

Frangou S, Modabbernia A, Doucet GE, Moser D, Dima D, ENIGMA Lifespan Working Group, (2020b): Cortical Thickness and Subcortical Volume Trajectories Across the Lifespan: Data From 14,600 Healthy Individuals Aged 6-90 Years. Biol Psychiatry 85:S35-S36. https://openaccess.city.ac.uk/id/eprint/22551/.

Frederiksen H, Johannsen TH, Andersen SE, Albrethsen J, Landersoe SK, Petersen JH, Andersen AN, Vestergaard ET, Schorring ME, Linneberg A, Main KM, Andersson A-M, Juul A (2020): Sex-specific Estrogen Levels and Reference Intervals from Infancy to Late Adulthood Determined by LC-MS/MS. J Clin Endocrinol Metab 105. http://dx.doi.org/10.1210/clinem/dgz196.

Freund A (2019): Untangling Aging Using Dynamic, Organism-Level Phenotypic Networks. Cell Syst 8:172-181. http://dx.doi.org/10.1016/j.cels.2019.02.005.

Fries GR, Bauer IE, Scaini G, Wu M-J, Kazimi IF, Valvassori SS, Zunta-Soares G, Walss-Bass C, Soares JC, Quevedo J (2017): Accelerated epigenetic aging and mitochondrial DNA copy number in bipolar disorder. Transl Psychiatry 7:1283. http://dx.doi.org/10.1038/s41398-017-0048-8.

Frodl T, Janowitz D, Schmaal L, Tozzi L, Dobrowolny H, Stein DJ, Veltman DJ, Wittfeld K, van Erp TGM, Jahanshad N, Block A, Hegenscheid K, Völzke H, Lagopoulos J, Hatton SN, Hickie IB, Frey EM, Carballedo A, Brooks SJ, Vuletic D, Uhlmann A, Veer IM, Walter H, Schnell K, Grotegerd D, Arolt V, Kugel H, Schramm E, Konrad C, Zurowski B, Baune BT, van der Wee NJA, van Tol M-J, Penninx BWJH, Thompson PM, Hibar DP, Dannlowski U, Grabe HJ (2017): Childhood adversity impacts on brain subcortical structures relevant to depression. J Psychiatr Res 86:58-65. http://dx.doi.org/10.1016/j.jpsychires.2016.11.010.

Fuentes N, Silveyra P (2019): Estrogen receptor signaling mechanisms. Adv Protein Chem Struct Biol 116:135-170. http://dx.doi.org/10.1016/bs.apcsb.2019.01.001.

Gee GC, Payne-Sturges DC (2004): Environmental health disparities: a framework integrating psychosocial and environmental concepts. Environ Health Perspect 112:1645-1653. http://dx.doi.org/10.1289/ehp.7074.

Geng D-Y, Li Y-X, Zee C-S (2006): Magnetic resonance imaging-based volumetric analysis of basal ganglia nuclei and substantia nigra in patients with Parkinson's disease. Neurosurgery 58:256-62; discussion 256-62. http://dx.doi.org/10.1227/01.NEU.0000194845.19462.7B.

Georgakis MK, Kalogirou EI, Diamantaras A-A, Daskalopoulou SS, Munro CA, Lyketsos CG, Skalkidou A, Petridou ET (2016): Age at menopause and duration of reproductive period in association with dementia and cognitive function: A systematic review and meta-analysis. Psychoneuroendocrinology 73:224-243. http://dx.doi.org/10.1016/j.psyneuen.2016.08.003.

Gimson A, Schlosser M, Huntley JD, Marchant NL (2018): Support for midlife anxiety diagnosis as an independent risk factor for dementia: a systematic review. BMJ Open 8:e019399. http://dx.doi.org/10.1136/bmjopen-2017-019399.

Glavak Tkalić R, Sučić I, Dević I (2013): Motivation for substance use: why do people use alcohol, tobacco and marijuana? Društvena istraživanja: časopis za opća društvena pitanja 22:601-625. https://hrcak.srce.hr/file/171167.

Golden LC, Voskuhl R (2017): The importance of studying sex differences in disease: The example of multiple sclerosis. J Neurosci Res 95:633-643. http://dx.doi.org/10.1002/jnr.23955.

Goldstein JM, Seidman LJ, O'Brien LM, Horton NJ, Kennedy DN, Makris N, Caviness VS Jr, Faraone SV, Tsuang MT (2002): Impact of normal sexual dimorphisms on sex differences in structural brain abnormalities in schizophrenia assessed by magnetic resonance imaging. Arch Gen Psychiatry 59:154-164. http://dx.doi.org/10.1001/archpsyc.59.2.154.

Goldstein RB, Smith SM, Chou SP, Saha TD, Jung J, Zhang H, Pickering RP, Ruan WJ, Huang B, Grant BF (2016): The epidemiology of DSM-5 posttraumatic stress disorder in the United States: results from the National Epidemiologic Survey on Alcohol and Related Conditions-III. Soc Psychiatry Psychiatr Epidemiol 51:1137-1148. http://dx.doi.org/10.1007/s00127-016-1208-5.

Goodman RA, Lochner KA, Thambisetty M, Wingo TS, Posner SF, Ling SM (2017): Prevalence of dementia subtypes in United States Medicare fee-for-service beneficiaries, 2011-2013. Alzheimers Dement 13:28-37. http://www.sciencedirect.com/science/article/pii/S1552526016300528.

Gottesman RF, Schneider ALC, Zhou Y, Chen X, Green E, Gupta N, Knopman DS, Mintz A, Rahmim A, Sharrett AR, Wagenknecht LE, Wong DF, Mosley TH Jr (2016): The ARIC-PET amyloid imaging study: Brain amyloid differences by age, race, sex, and APOE. Neurology 87:473-480. 
http://dx.doi.org/10.1212/WNL.0000000000002914.

Grace S, Rossetti MG, Allen N, Batalla A, Bellani M, Brambilla P, Chye Y, Cousijn J, Goudriaan A, Hester R, Hutchison K, Labuschagne I, Li CR, Reza Momenan R, Martin-Santos R, Rendell P, Solowij N, Sinha R, Schmaal L, Sjoerds Z, Suo C, Terrett G, van Holst R, Veltman D, Yücel M, Thompson P, Conrod P, Mackey S, Garavan H, Lorenzettl V (2020): Sex differences in the neuroanatomy of alcohol dependence: hippocampus and amygdala Subregions in a sample of 966 people from the ENIGMA Addiction Working Group. Transl Psychiatry.

Grant BF, Goldstein RB, Chou SP, Huang B, Stinson FS, Dawson DA, Saha TD, Smith SM, Pulay AJ, Pickering RP, Ruan WJ, Compton WM (2009): Sociodemographic and psychopathologic predictors of first incidence of DSM-IV substance use, mood and anxiety disorders: results from the Wave 2 National Epidemiologic Survey on Alcohol and Related Conditions. Mol Psychiatry 14:1051-1066. https://doi.org/10.1038/mp.2008.41.

Greenfield SF, Back SE, Lawson K, Brady KT (2010): Substance abuse in women. Psychiatr Clin North Am 33:339-355. http://dx.doi.org/10.1016/j.psc.2010.01.004.

Grossman LS, Harrow M, Rosen C, Faull R (2006): Sex differences in outcome and recovery for schizophrenia and other psychotic and nonpsychotic disorders. Psychiatr Serv 57:844-850. http://dx.doi.org/10.1176/ps.2006.57.6.844.

Grossman LS, Harrow M, Rosen C, Faull R, Strauss GP (2008): Sex differences in schizophrenia and other psychotic disorders: a 20-year longitudinal study of psychosis and recovery. Compr Psychiatry 49:523-529. http://dx.doi.org/10.1016/j.comppsych.2008.03.004.

Guadalupe T, Mathias SR, vanErp TGM, Whelan CD, Zwiers MP, Abe Y, Abramovic L, Agartz I, Andreassen OA, Arias-Vásquez A, Aribisala BS, Armstrong NJ, Arolt V, Artiges E, Ayesa-Arriola R, Baboyan VG, Banaschewski T, Barker G, Bastin ME, Baune BT, Blangero J, Bokde ALW, Boedhoe PSW, Bose A, Brem S, Brodaty H, Bromberg U, Brooks S, Büchel C, Buitelaar J, Calhoun VD, Cannon DM, Cattrell A, Cheng Y, Conrod PJ, Conzelmann A, Corvin A, Crespo-Facorro B, Crivello F, Dannlowski U, de Zubicaray GI, de Zwarte SMC, Deary IJ, Desrivières S, Doan NT, Donohoe G, Dørum ES, Ehrlich S, Espeseth T, Fernández G, Flor H, Fouche J-P, Frouin V, Fukunaga M, Gallinat J, Garavan H, Gill M, Suarez AG, Gowland P, Grabe HJ, Grotegerd D, Gruber O, Hagenaars S, Hashimoto R, Hauser TU, Heinz A, Hibar DP, Hoekstra PJ, Hoogman M, Howells FM, Hu H, Hulshoff Pol HE, Huyser C, Ittermann B, Jahanshad N, Jönsson EG, Jurk S, Kahn RS, Kelly S, Kraemer B, Kugel H, Kwon JS, Lemaitre H, Lesch K-P, Lochner C, Luciano M, Marquand AF, Martin NG, Martínez-Zalacaín I, Martinot J-L, Mataix-Cols D, Mather K, McDonald C, McMahon KL, Medland SE, Menchón JM, Morris DW, Mothersill O, Maniega SM, Mwangi B, Nakamae T, Nakao T, Narayanaswaamy JC, Nees F, Nordvik JE, Onnink AMH, Opel N, Ophoff R, Paillère Martinot M-L, Papadopoulos Orfanos D, Pauli P, Paus T, Poustka L, Reddy JY, Renteria ME, Roiz-Santiáñez R, Roos A, Royle NA, Sachdev P, Sánchez-Juan P, Schmaal L, Schumann G, Shumskaya E, Smolka MN, Soares JC, Soriano-Mas C, Stein DJ, Strike LT, Toro R, Turner JA, Tzourio-Mazoyer N, Uhlmann A, Hernández MV, van den Heuvel OA, van der Meer D, van Haren NEM, Veltman DJ, Venkatasubramanian G, Vetter NC, Vuletic D, Walitza S, Walter H, Walton E, Wang Z, Wardlaw J, Wen W, Westlye LT, Whelan R, Wittfeld K, Wolfers T, Wright MJ, Xu J, Xu X, Yun J-Y, Zhao J, Franke B, Thompson PM, Glahn DC, Mazoyer B, Fisher SE, Francks C (2017): Human subcortical brain asymmetries in 15,847 people worldwide reveal effects of age and sex. Brain Imaging Behav 11:1497-1514. http://dx.doi.org/10.1007/s11682-016-9629-z.

Guerreiro R, Bras J (2015): The age factor in Alzheimer's disease. Genome Med 7:106. http://dx.doi.org/10.1186/s13073-015-0232-5.

Gutman B, Bright J, Rummel C, Rocha CS, Debove I, Yasuda C, Guimaraes RP, Bergo F, D'Abreu A, Poston K, Wiest R, Cendes F, Thompson PM, van der Werf Y (2018): Widespread Cortical Thinning in Parkinson's Disease: Findings from the ENIGMA-Parkinson's Disease Working Group (S45.008). Neurology 90. https://n.neurology.org/content/90/15_Supplement/S45.008.abstract.

Haaxma CA, Bloem BR, Borm GF, Oyen WJG, Leenders KL, Eshuis S, Booij J, Dluzen DE, Horstink MWIM (2007): Gender differences in Parkinson's disease. J Neurol Neurosurg Psychiatry 78:819_ 824. http://dx.doi.org/10.1136/jnnp.2006.103788.

Haider A, Spurling BC, Sánchez-Manso JC (2020): Lewy Body Dementia. In: . StatPearls. Treasure Island (FL): StatPearls Publishing. https://www.ncbi.nlm.nih.gov/pubmed/29494048.

Hajek T, Franke K, Kolenic M, Capkova J, Matejka M, Propper L, Uher R, Stopkova P, Novak T, Paus T, Kopecek M, Spaniel F, Alda M (2019): Brain Age in Early Stages of Bipolar Disorders or 
Schizophrenia. Schizophr Bull 45:190-198. http://dx.doi.org/10.1093/schbul/sbx172.

Hamidullah S, Thorpe HHA, Frie JA, Mccurdy RD, Khokhar JY (2020): Adolescent Substance Use and the Brain: Behavioral, Cognitive and Neuroimaging Correlates. Front Hum Neurosci 14:298. http://dx.doi.org/10.3389/fnhum.2020.00298.

Handelsman DJ, Sikaris K, Ly LP (2016): Estimating age-specific trends in circulating testosterone and sex hormone-binding globulin in males and females across the lifespan. Ann Clin Biochem 53:377384. http://dx.doi.org/10.1177/0004563215610589.

Han LKM, Dinga R, Hahn T, Ching C, Eyler L, Aftanas L, Aghajani M, Aleman A, Baune B, Berger K, Brak I, Filho GB, Carballedo A, Connolly C, Couvy-Duchesne B, Cullen K, Dannlowski U, Davey C, Dima D, Duran F, Enneking V, Filimonova E, Frenzel S, Frodl T, Fu C, Godlewska B, Gotlib I, Grabe H, Groenewold N, Grotegerd D, Gruber O, Hall G, Harrison B, Hatton S, Hermesdorf M, Hickie I, Ho T, Hosten N, Jansen A, Kahler C, Kircher T, Klimes-Dougan B, Kramer B, Krug A, Lagopoulos J, Leenings R, MacMaster F, MacQueen G, Mclntosh A, McLellan Q, McMahon K, Medland S, Mueller B, Mwangi B, Osipov E, Portella M, Pozzi E, Reneman L, Repple J, Rosa P, Sacchet M, Saemann P, Schnell K, Schrantee A, Simulionyte E, Soares J, Sommer J, Stein D, Steinstrater O, Strike L, Thomopoulos S, van Tol M-J, Veer I, Vermeiren R, Walter H, van der Wee N, van der Werff S, Whalley H, Winter N, Wittfeld K, Wright M, Wu M-J, Volzke H, Yang T, Zannias V, de Zubicaray G, Zunta-Soares G, Abe C, Alda M, Andreassen O, Boen E, Bonnin C, Canales-Rodriguez E, Cannon D, Caseras X, Chaim-Avancini T, Elvsashagen T, Favre P, Foley S, Fullerton J, Goikolea J, Haarman B, Hajek T, Henry C, Houenou J, Howells F, Ingvar M, Kuplicki R, Lafer B, Landen M, MachadoVieira R, Malt U, McDonald C, Mitchell P, Nabulsi L, Otaduy M, Overs B, Polosan M, Pomarol-Clotet E, Radua J, Rive M, Roberts G, Ruhe H, Salvador R, Sarro S, Satterthwaite T, Savitz J, Schene A, Schofield P, Serpa M, Sim K, Soeiro-de-Souza M, Sutherland A, Temmingh H, Timmons G, Uhlmann A, Vieta E, Wolf D, Zanetti M, Jahanshad N, Thompson P, Veltman D, Penninx B, Marquand A, Cole J, Schmaal L (2019): Brain Aging in Major Depressive Disorder: Results from the ENIGMA Major Depressive Disorder working group. bioRxiv. https://www.biorxiv.org/content/10.1101/560623v1.

Hansen M, Schoonover A, Skarica B, Harrod T, Bahr N, Guise J-M (2019): Implicit gender bias among US resident physicians. BMC Med Educ 19:396. http://dx.doi.org/10.1186/s12909-019-1818-1.

Harman SM, Metter EJ, Tobin JD, Pearson J, Blackman MR, Baltimore Longitudinal Study of Aging (2001): Longitudinal effects of aging on serum total and free testosterone levels in healthy men. Baltimore Longitudinal Study of Aging. J Clin Endocrinol Metab 86:724-731. http://dx.doi.org/10.1210/jcem.86.2.7219.

Hauer L, Perneczky J, Sellner J (2020): A global view of comorbidity in multiple sclerosis: a systematic review with a focus on regional differences, methodology, and clinical implications. J Neurol. http://dx.doi.org/10.1007/s00415-020-10107-y.

Hawco C, Voineskos AN, Radhu N, Rotenberg D, Ameis S, Backhouse FA, Semeralul M, Daskalakis ZJ (2017): Age and gender interactions in white matter of schizophrenia and obsessive compulsive disorder compared to non-psychiatric controls: commonalities across disorders. Brain Imaging Behav 11:1836-1848.

https://idp.springer.com/authorize/casa?redirect_uri=https://link.springer.com/article/10.1007/s11682016-9657-

8\&casa_token=wXzp30qMkMMAAAAA:w5Zl8ZIfCzPZHMSQA4BzvfidMPUxCk88WVYFDDMfQxNzP Bp4iJI9dYAXYCRDxvPRTAeztekLNJ0-MbMBnw.

Hazari N, Narayanaswamy JC, Venkatasubramanian G (2019): Neuroimaging findings in obsessivecompulsive disorder: A narrative review to elucidate neurobiological underpinnings. Indian $\mathrm{J}$ Psychiatry 61:S9-S29. http://dx.doi.org/10.4103/psychiatry.IndianJPsychiatry_525_18.

Hebert LE, Weuve J, Scherr PA, Evans DA (2013): Alzheimer disease in the United States (2010-2050) estimated using the 2010 census. Neurology 80:1778-1783. http://dx.doi.org/10.1212/WNL.0b013e31828726f5.

Heller C, Balls-Berry JE, Nery JD, Erwin PJ, Littleton D, Kim M, Kuo WP (2014): Strategies addressing barriers to clinical trial enrollment of underrepresented populations: a systematic review. Contemp Clin Trials 39:169-182. http://dx.doi.org/10.1016/j.cct.2014.08.004.

Henderson VW, St John JA, Hodis HN, McCleary CA, Stanczyk FZ, Shoupe D, Kono N, Dustin L, Allayee H, Mack WJ (2016): Cognitive effects of estradiol after menopause: A randomized trial of the timing hypothesis. Neurology 87:699-708. http://dx.doi.org/10.1212/WNL.0000000000002980.

Herting MM, Sowell ER (2017): Puberty and structural brain development in humans. Front 
Neuroendocrinol 44:122-137. http://dx.doi.org/10.1016/j.yfrne.2016.12.003.

Heyrana K, Byers HM, Stratton P (2018): Increasing the Participation of Pregnant Women in Clinical Trials. JAMA 320:2077-2078. http://dx.doi.org/10.1001/jama.2018.17716.

Hibar DP, Westlye LT, Doan NT, Jahanshad N, Cheung JW, Ching CRK, Versace A, Bilderbeck AC, Uhlmann A, Mwangi B, Krämer B, Overs B, Hartberg CB, Abé C, Dima D, Grotegerd D, Sprooten E, Bøen E, Jimenez E, Howells FM, Delvecchio G, Temmingh H, Starke J, Almeida JRC, Goikolea JM, Houenou J, Beard LM, Rauer L, Abramovic L, Bonnin M, Ponteduro MF, Keil M, Rive MM, Yao N, Yalin N, Najt P, Rosa PG, Redlich R, Trost S, Hagenaars S, Fears SC, Alonso-Lana S, van Erp TGM, Nickson T, Chaim-Avancini TM, Meier TB, Elvsåshagen T, Haukvik UK, Lee WH, Schene AH, Lloyd AJ, Young AH, Nugent A, Dale AM, Pfennig A, Mclntosh AM, Lafer B, Baune BT, Ekman CJ, Zarate CA, Bearden CE, Henry C, Simhandl C, McDonald C, Bourne C, Stein DJ, Wolf DH, Cannon DM, Glahn DC, Veltman DJ, Pomarol-Clotet E, Vieta E, Canales-Rodriguez EJ, Nery FG, Duran FLS, Busatto GF, Roberts G, Pearlson GD, Goodwin GM, Kugel H, Whalley HC, Ruhe HG, Soares JC, Fullerton JM, Rybakowski JK, Savitz J, Chaim KT, Fatjó-Vilas M, Soeiro-de-Souza MG, Boks MP, Zanetti MV, Otaduy MCG, Schaufelberger MS, Alda M, Ingvar M, Phillips ML, Kempton MJ, Bauer M, Landén M, Lawrence NS, van Haren NEM, Horn NR, Freimer NB, Gruber O, Schofield PR, Mitchell PB, Kahn RS, Lenroot R, Machado-Vieira R, Ophoff RA, Sarró S, Frangou S, Satterthwaite TD, Hajek T, Dannlowski U, Malt UF, Arolt V, Gattaz WF, Drevets WC, Caseras X, Agartz I, Thompson PM, Andreassen OA (2018): Cortical abnormalities in bipolar disorder: an MRI analysis of 6503 individuals from the ENIGMA Bipolar Disorder Working Group. Mol Psychiatry 23:932-942. http://dx.doi.org/10.1038/mp.2017.73.

Hibar DP, Westlye LT, van Erp TGM, Rasmussen J, Leonardo CD, Faskowitz J, Haukvik UK, Hartberg CB, Doan NT, Agartz I, Dale AM, Gruber O, Krämer B, Trost S, Liberg B, Abé C, Ekman CJ, Ingvar M, Landén M, Fears SC, Freimer NB, Bearden CE, Costa Rica/Colombia Consortium for Genetic Investigation of Bipolar Endophenotypes, Sprooten E, Glahn DC, Pearlson GD, Emsell L, Kenney J, Scanlon C, McDonald C, Cannon DM, Almeida J, Versace A, Caseras X, Lawrence NS, Phillips ML, Dima D, Delvecchio G, Frangou S, Satterthwaite TD, Wolf D, Houenou J, Henry C, Malt UF, Bøen E, Elvsåshagen T, Young AH, Lloyd AJ, Goodwin GM, Mackay CE, Bourne C, Bilderbeck A, Abramovic L, Boks MP, van Haren NEM, Ophoff RA, Kahn RS, Bauer M, Pfennig A, Alda M, Hajek T, Mwangi B, Soares JC, Nickson T, Dimitrova R, Sussmann JE, Hagenaars S, Whalley HC, McIntosh AM, Thompson PM, Andreassen OA (2016): Subcortical volumetric abnormalities in bipolar disorder. Mol Psychiatry 21:1710-1716. http://dx.doi.org/10.1038/mp.2015.227.

Higgins JJ, Mendez MF (2000): Roll over Pick and tell Alzheimer the news! Neurology. AAN Enterprises. http://dx.doi.org/10.1212/wnl.54.4.784.

Honnorat N, Dong A, Meisenzahl-Lechner E, Koutsouleris N, Davatzikos C (2019): Neuroanatomical heterogeneity of schizophrenia revealed by semi-supervised machine learning methods. Schizophr Res 214:43-50. http://dx.doi.org/10.1016/j.schres.2017.12.008.

Howard G, Cushman M, Moy CS, Oparil S, Muntner P, Lackland DT, Manly JJ, Flaherty ML, Judd SE, Wadley VG, Long DL, Howard VJ (2018): Association of Clinical and Social Factors With Excess Hypertension Risk in Black Compared With White US Adults. JAMA 320:1338-1348. http://dx.doi.org/10.1001/jama.2018.13467.

Howard G, Safford MM, Moy CS, Howard VJ, Kleindorfer DO, Unverzagt FW, Soliman EZ, Flaherty ML, McClure LA, Lackland DT, Wadley VG, Pulley L, Cushman M (2017): Racial Differences in the Incidence of Cardiovascular Risk Factors in Older Black and White Adults. J Am Geriatr Soc 65:8390. http://dx.doi.org/10.1111/jgs.14472.

Hua X, Hibar DP, Lee S, Toga AW, Jack CR Jr, Weiner MW, Thompson PM, Alzheimer's Disease Neuroimaging Initiative (2010): Sex and age differences in atrophic rates: an ADNI study with $n=1368$ MRI scans. Neurobiol Aging 31:1463-1480. http://dx.doi.org/10.1016/j.neurobiolaging.2010.04.033.

Huey ED, Putnam KT, Grafman J (2006): A systematic review of neurotransmitter deficits and treatments in frontotemporal dementia. Neurology 66:17-22. http://dx.doi.org/10.1212/01.wnl.0000191304.55196.4d.

Hu J, Zhang Z, Shen W-J, Azhar S (2010): Cellular cholesterol delivery, intracellular processing and utilization for biosynthesis of steroid hormones. Nutr Metab 7:47. http://dx.doi.org/10.1186/17437075-7-47.

Hussain-Gambles M, Atkin K, Leese B (2004): Why ethnic minority groups are under-represented in clinical trials: a review of the literature. Health Soc Care Community 12:382-388. 
http://dx.doi.org/10.1111/j.1365-2524.2004.00507.x.

Huxley RR, Woodward M (2011): Cigarette smoking as a risk factor for coronary heart disease in women compared with men: a systematic review and meta-analysis of prospective cohort studies. Lancet 378:1297-1305. http://dx.doi.org/10.1016/S0140-6736(11)60781-2.

Hyatt CS, Owens MM, Crowe ML, Carter NT, Lynam DR, Miller JD (2020): The quandary of covarying: A brief review and empirical examination of covariate use in structural neuroimaging studies on psychological variables. Neuroimage 205:116225. http://dx.doi.org/10.1016/j.neuroimage.2019.116225.

Hyman BT, Van Hoesen GW, Damasio AR, Barnes CL (1984): Alzheimer's disease: cell-specific pathology isolates the hippocampal formation. Science 225:1168-1170. http://dx.doi.org/10.1126/science.6474172.

Iglesias JE, Augustinack JC, Nguyen K, Player CM, Player A, Wright M, Roy N, Frosch MP, McKee AC, Wald LL, Fischl B, Van Leemput K, Alzheimer's Disease Neuroimaging Initiative (2015): A computational atlas of the hippocampal formation using ex vivo, ultra-high resolution MRI: Application to adaptive segmentation of in vivo MRI. Neuroimage 115:117-137. http://dx.doi.org/10.1016/j.neuroimage.2015.04.042.

Imtiaz B, Tuppurainen M, Rikkonen T, Kivipelto M, Soininen H, Kröger H, Tolppanen A-M (2017): Postmenopausal hormone therapy and Alzheimer disease: A prospective cohort study. Neurology 88:1062-1068. http://dx.doi.org/10.1212/WNL.0000000000003696.

Insel T, Cuthbert B, Garvey M, Heinssen R, Pine DS, Quinn K, Sanislow C, Wang P (2010): Research domain criteria (RDoC): toward a new classification framework for research on mental disorders. Am J Psychiatry 167:748-751. http://dx.doi.org/10.1176/appi.ajp.2010.09091379.

Inslicht SS, Metzler TJ, Garcia NM, Pineles SL, Milad MR, Orr SP, Marmar CR, Neylan TC (2013): Sex differences in fear conditioning in posttraumatic stress disorder. J Psychiatr Res 47:64-71. http://dx.doi.org/10.1016/j.jpsychires.2012.08.027.

Institute for Health Metrics and Evaluation (IHME) (2019): GBD Compare Data Visualization. Global Burden of Disease. http://vizhub.healthdata.org/gbd-compare.

loannidis JPA (2005a): Contradicted and initially stronger effects in highly cited clinical research. JAMA 294:218-228. http://dx.doi.org/10.1001/jama.294.2.218.

loannidis JPA (2005b): Why most published research findings are false. PLoS Med 2:e124. http://dx.doi.org/10.1371/journal.pmed.0020124.

loannidis JPA (2008): Why most discovered true associations are inflated. Epidemiology 19:640-648. http://dx.doi.org/10.1097/EDE.0b013e31818131e7.

loannidis JPA (2011): Excess significance bias in the literature on brain volume abnormalities. Arch Gen Psychiatry 68:773-780. http://dx.doi.org/10.1001/archgenpsychiatry.2011.28.

Irish LA, Fischer B, Fallon W, Spoonster E, Sledjeski EM, Delahanty DL (2011): Gender differences in PTSD symptoms: an exploration of peritraumatic mechanisms. J Anxiety Disord 25:209-216. http://dx.doi.org/10.1016/j.janxdis.2010.09.004.

Ishibashi M, Kimura N, Sumi K, Aso Y, Matsubara E (2019): Comparison of brain perfusion patterns in dementia with Lewy bodies patients with or without cingulate island sign. Geriatr Gerontol Int 19:197202. http://dx.doi.org/10.1111/ggi.13586.

Jack CR, Bennett DA, Blennow K, Carrillo MC, Dunn B, Haeberlein SB, Holtzman DM, Jagust W, Jessen F, Karlawish J, Liu E, Molinuevo JL, Montine T, Phelps C, Rankin KP, Rowe CC, Scheltens P, Siemers E, Snyder HM, Sperling R, Elliott C, Masliah E, Ryan L, Silverberg N (2018a): NIA-AA Research Framework: Toward a biological definition of Alzheimer's disease. Alzheimer's \& Dementia. http://dx.doi.org/10.1016/j.jalz.2018.02.018.

Jack CR Jr, Bennett DA, Blennow K, Carrillo MC, Dunn B, Haeberlein SB, Holtzman DM, Jagust W, Jessen F, Karlawish J, Others (2018b): NIA-AA research framework: toward a biological definition of Alzheimer's disease. Alzheimers Dement 14:535-562. https://alzjournals.onlinelibrary.wiley.com/doi/abs/10.1016/j.jalz.2018.02.018.

Jack CR Jr, Petersen RC, Xu YC, Waring SC, O'Brien PC, Tangalos EG, Smith GE, Ivnik RJ, Kokmen E (1997): Medial temporal atrophy on MRI in normal aging and very mild Alzheimer's disease. Neurology 49:786-794. http://dx.doi.org/10.1212/wnl.49.3.786.

Jahanshad N, Thompson PM (2017): Multimodal neuroimaging of male and female brain structure in health and disease across the life span. J Neurosci Res 95:371-379. http://dx.doi.org/10.1002/jnr.23919. 
Jakimovski D, Zivadinov R, Bergsland N, Ramasamy DP, Hagemeier J, Weinstock-Guttman B, Kolb C, Hojnacki D, Dwyer MG (2020): Sex-Specific Differences in Life Span Brain Volumes in Multiple Sclerosis. J Neuroimaging 30:342-350. https://onlinelibrary.wiley.com/doi/abs/10.1111/jon.12709.

Jalnapurkar I, Allen M, Pigott T (2018): Sex differences in anxiety disorders: A review. J Psychiatry Depress Anxiety 4:012.

https://www.researchgate.net/profile/lsha_Jalnapurkar/publication/323847924_Sex_Differences_in_A nxiety_Disorders_A_Review/links/5aaf8f09458515ecebe9bc1c/Sex-Differences-in-Anxiety-DisordersA-Review.pdf.

James SL, Abate D, Abate KH, Abay SM, Abbafati C, Abbasi N, Abbastabar H, Abd-Allah F, Abdela J, Abdelalim A, Abdollahpour I, Abdulkader RS, Abebe Z, Abera SF, Abil OZ, Abraha HN, Abu-Raddad LJ, Abu-Rmeileh NME, Accrombessi MMK, Acharya D, Acharya P, Ackerman IN, Adamu AA, Adebayo OM, Adekanmbi V, Adetokunboh OO, Adib MG, Adsuar JC, Afanvi KA, Afarideh M, Afshin A, Agarwal G, Agesa KM, Aggarwal R, Aghayan SA, Agrawal S, Ahmadi A, Ahmadi M, Ahmadieh H, Ahmed MB, Aichour AN, Aichour I, Aichour MTE, Akinyemiju T, Akseer N, Al-Aly Z, Al-Eyadhy A, AlMekhlafi HM, Al-Raddadi RM, Alahdab F, Alam K, Alam T, Alashi A, Alavian SM, Alene KA, Alijanzadeh M, Alizadeh-Navaei R, Aljunid SM, Alkerwi A 'a, Alla F, Allebeck P, Alouani MML, Altirkawi K, Alvis-Guzman N, Amare AT, Aminde LN, Ammar W, Amoako YA, Anber NH, Andrei CL, Androudi S, Animut MD, Anjomshoa M, Ansha MG, Antonio CAT, Anwari P, Arabloo J, Arauz A, Aremu O, Ariani F, Armoon B, Ärnlöv J, Arora A, Artaman A, Aryal KK, Asayesh H, Asghar RJ, Ataro Z, Atre SR, Ausloos M, Avila-Burgos L, Avokpaho EFGA, Awasthi A, Ayala Quintanilla BP, Ayer R, Azzopardi PS, Babazadeh A, Badali H, Badawi A, Bali AG, Ballesteros KE, Ballew SH, Banach M, Banoub JAM, Banstola A, Barac A, Barboza MA, Barker-Collo SL, Bärnighausen TW, Barrero LH, Baune BT, Bazargan-Hejazi S, Bedi N, Beghi E, Behzadifar M, Behzadifar M, Béjot Y, Belachew AB, Belay YA, Bell ML, Bello AK, Bensenor IM, Bernabe E, Bernstein RS, Beuran M, Beyranvand T, Bhala N, Bhattarai S, Bhaumik S, Bhutta ZA, Biadgo B, Bijani A, Bikbov B, Bilano V, Bililign N, Bin Sayeed MS, Bisanzio D, Blacker BF, Blyth FM, Bou-Orm IR, Boufous S, Bourne R, Brady OJ, Brainin M, Brant LC, Brazinova A, Breitborde NJK, Brenner H, Briant PS, Briggs AM, Briko AN, Britton G, Brugha T, Buchbinder R, Busse R, Butt ZA, Cahuana-Hurtado L, Cano J, Cárdenas R, Carrero JJ, Carter A, Carvalho F, Castañeda-Orjuela CA, Castillo Rivas J, Castro F, Catalá-López F, Cercy KM, Cerin E, Chaiah Y, Chang AR, Chang H-Y, Chang J-C, Charlson FJ, Chattopadhyay A, Chattu VK, Chaturvedi P, Chiang PP-C, Chin KL, Chitheer A, Choi J-YJ, Chowdhury R, Christensen H, Christopher DJ, Cicuttini FM, Ciobanu LG, Cirillo M, Claro RM, Collado-Mateo D, Cooper C, Coresh J, Cortesi PA, Cortinovis M, Costa M, Cousin E, Criqui MH, Cromwell EA, Cross M, Crump JA, Dadi AF, Dandona L, Dandona R, Dargan PI, Daryani A, Das Gupta R, Das Neves J, Dasa TT, Davey G, Davis AC, Davitoiu DV, De Courten B, De La Hoz FP, De Leo D, De Neve J-W, Degefa MG, Degenhardt L, Deiparine S, Dellavalle RP, Demoz GT, Deribe K, Dervenis N, Des Jarlais DC, Dessie GA, Dey S, Dharmaratne SD, Dinberu MT, Dirac MA, Djalalinia S, Doan L, Dokova K, Doku DT, Dorsey ER, Doyle KE, Driscoll TR, Dubey M, Dubljanin E, Duken EE, Duncan BB, Duraes AR, Ebrahimi H, Ebrahimpour S, Echko MM, Edvardsson D, Effiong A, Ehrlich JR, El Bcheraoui C, El Sayed Zaki M, El-Khatib Z, Elkout H, Elyazar IRF, Enayati A, Endries AY, Er B, Erskine HE, Eshrati B, Eskandarieh S, Esteghamati A, Esteghamati S, Fakhim H, Fallah Omrani V, Faramarzi M, Fareed M, Farhadi F, Farid TA, Farinha CSE sá, Farioli A, Faro A, Farvid MS, Farzadfar F, Feigin VL, Fentahun N, Fereshtehnejad S-M, Fernandes E, Fernandes JC, Ferrari AJ, Feyissa GT, Filip I, Fischer F, Fitzmaurice C, Foigt NA, Foreman KJ, Fox J, Frank TD, Fukumoto T, Fullman N, Fürst T, Furtado JM, Futran ND, Gall S, Ganji M, Gankpe FG, Garcia-Basteiro AL, Gardner WM, Gebre AK, Gebremedhin AT, Gebremichael TG, Gelano TF, Geleijnse JM, Genova-Maleras R, Geramo YCD, Gething PW, Gezae KE, Ghadiri K, Ghasemi Falavarjani K, Ghasemi-Kasman M, Ghimire M, Ghosh R, Ghoshal AG, Giampaoli S, Gill PS, Gill TK, Ginawi IA, Giussani G, Gnedovskaya EV, Goldberg EM, Goli S, Gómez-Dantés H, Gona PN, Gopalani SV, Gorman TM, Goulart AC, Goulart BNG, Grada A, Grams ME, Grosso G, Gugnani HC, Guo Y, Gupta PC, Gupta R, Gupta R, Gupta T, Gyawali B, Haagsma JA, Hachinski V, Hafezi-Nejad N, Haghparast Bidgoli H, Hagos TB, Hailu GB, Haj-Mirzaian A, Haj-Mirzaian A, Hamadeh RR, Hamidi S, Handal AJ, Hankey GJ, Hao Y, Harb HL, Harikrishnan S, Haro JM, Hasan M, Hassankhani H, Hassen HY, Havmoeller R, Hawley CN, Hay RJ, Hay SI, Hedayatizadeh-Omran A, Heibati B, Hendrie D, Henok A, Herteliu C, Heydarpour S, Hibstu DT, Hoang HT, Hoek HW, Hoffman HJ, Hole MK, Homaie Rad E, Hoogar P, Hosgood HD, Hosseini SM, Hosseinzadeh M, Hostiuc M, Hostiuc S, Hotez PJ, Hoy DG, Hsairi M, Htet AS, Hu G, Huang JJ, 
Huynh CK, Iburg KM, Ikeda CT, Ileanu B, Ilesanmi OS, Iqbal U, Irvani SSN, Irvine CMS, Islam SMS, Islami $F$, Jacobsen $K H$, Jahangiry $L$, Jahanmehr $N$, Jain $S K$, Jakovljevic $M$, Javanbakht $M$, Jayatilleke AU, Jeemon P, Jha RP, Jha V, Ji JS, Johnson CO, Jonas JB, Jozwiak JJ, Jungari SB, Jürisson M, Kabir Z, Kadel R, Kahsay A, Kalani R, Kanchan T, Karami M, Karami Matin B, Karch A, Karema C, Karimi N, Karimi SM, Kasaeian A, Kassa DH, Kassa GM, Kassa TD, Kassebaum NJ, Katikireddi SV, Kawakami N, Karyani AK, Keighobadi MM, Keiyoro PN, Kemmer L, Kemp GR, Kengne AP, Keren A, Khader YS, Khafaei B, Khafaie MA, Khajavi A, Khalil IA, Khan EA, Khan MS, Khan MA, Khang Y-H, Khazaei M, Khoja AT, Khosravi A, Khosravi MH, Kiadaliri AA, Kiirithio DN, Kim C-I, Kim D, Kim P, Kim Y-E, Kim YJ, Kimokoti RW, Kinfu Y, Kisa A, Kissimova-Skarbek K, Kivimäki M, Knudsen AKS, Kocarnik JM, Kochhar S, Kokubo Y, Kolola T, Kopec JA, Kosen S, Kotsakis GA, Koul PA, Koyanagi A, Kravchenko MA, Krishan K, Krohn KJ, Kuate Defo B, Kucuk Bicer B, Kumar GA, Kumar M, Kyu HH, Lad DP, Lad SD, Lafranconi A, Lalloo R, Lallukka T, Lami FH, Lansingh VC, Latifi A, Lau KM-M, Lazarus JV, Leasher JL, Ledesma JR, Lee PH, Leigh J, Leung J, Levi M, Lewycka S, Li S, Li Y, Liao Y, Liben ML, Lim L-L, Lim SS, Liu S, Lodha R, Looker KJ, Lopez AD, Lorkowski S, Lotufo PA, Low N, Lozano R, Lucas TCD, Lucchesi LR, Lunevicius R, Lyons RA, Ma S, Macarayan ERK, Mackay MT, Madotto F, Magdy Abd El Razek H, Magdy Abd El Razek M, Maghavani DP, Mahotra NB, Mai HT, Majdan M, Majdzadeh R, Majeed A, Malekzadeh R, Malta DC, Mamun AA, Manda A-L, Manguerra H, Manhertz T, Mansournia MA, Mantovani LG, Mapoma CC, Maravilla JC, Marcenes W, Marks A, Martins-Melo FR, Martopullo I, März W, Marzan MB, Mashamba-Thompson TP, Massenburg BB, Mathur MR, Matsushita K, Maulik PK, Mazidi M, McAlinden C, McGrath JJ, McKee M, Mehndiratta MM, Mehrotra R, Mehta KM, Mehta V, Mejia-Rodriguez F, Mekonen T, Melese A, Melku M, Meltzer M, Memiah PTN, Memish ZA, Mendoza W, Mengistu DT, Mengistu G, Mensah GA, Mereta ST, Meretoja A, Meretoja TJ, Mestrovic T, Mezerji NMG, Miazgowski B, Miazgowski T, Millear Al, Miller TR, Miltz B, Mini GK, Mirarefin M, Mirrakhimov EM, Misganaw AT, Mitchell PB, Mitiku H, Moazen B, Mohajer B, Mohammad KA, Mohammadifard N, Mohammadnia-Afrouzi M, Mohammed MA, Mohammed S, Mohebi F, Moitra M, Mokdad AH, Molokhia M, Monasta L, Moodley Y, Moosazadeh M, Moradi G, Moradi-Lakeh M, Moradinazar M, Moraga P, Morawska L, Moreno Velásquez I, Morgado-Da-Costa J, Morrison SD, Moschos MM, Mountjoy-Venning WC, Mousavi SM, Mruts KB, Muche AA, Muchie KF, Mueller UO, Muhammed OS, Mukhopadhyay S, Muller K, Mumford JE, Murhekar M, Musa J, Musa KI, Mustafa G, Nabhan AF, Nagata C, Naghavi M, Naheed A, Nahvijou A, Naik G, Naik N, Najafi F, Naldi L, Nam HS, Nangia V, Nansseu JR, Nascimento BR, Natarajan G, Neamati N, Negoi I, Negoi RI, Neupane S, Newton CRJ, Ngunjiri JW, Nguyen AQ, Nguyen HT, Nguyen HLT, Nguyen HT, Nguyen LH, Nguyen M, Nguyen NB, Nguyen SH, Nichols E, Ningrum DNA, Nixon MR, Nolutshungu N, Nomura S, Norheim OF, Noroozi M, Norrving B, Noubiap JJ, Nouri HR, Nourollahpour Shiadeh M, Nowroozi MR, Nsoesie EO, Nyasulu PS, Odell CM, Ofori-Asenso R, Ogbo FA, Oh I-H, Oladimeji O, Olagunju AT, Olagunju TO, Olivares PR, Olsen HE, Olusanya BO, Ong KL, Ong SK, Oren E, Ortiz A, Ota E, Otstavnov SS, Øverland S, Owolabi MO, P A M, Pacella R, Pakpour AH, Pana A, Panda-Jonas S, Parisi A, Park E-K, Parry CDH, Patel S, Pati S, Patil ST, Patle A, Patton GC, Paturi VR, Paulson KR, Pearce N, Pereira DM, Perico N, Pesudovs K, Pham HQ, Phillips MR, Pigott DM, Pillay JD, Piradov MA, Pirsaheb M, Pishgar F, Plana-Ripoll O, Plass D, Polinder S, Popova S, Postma MJ, Pourshams A, Poustchi H, Prabhakaran D, Prakash S, Prakash V, Purcell CA, Purwar MB, Qorbani M, Quistberg DA, Radfar A, Rafay A, Rafiei A, Rahim F, Rahimi K, Rahimi-Movaghar A, Rahimi-Movaghar V, Rahman M, Rahman MH ur, Rahman MA, Rahman SU, Rai RK, Rajati F, Ram U, Ranjan P, Ranta A, Rao PC, Rawaf DL, Rawaf S, Reddy KS, Reiner RC, Reinig N, Reitsma MB, Remuzzi G, Renzaho AMN, Resnikoff S, Rezaei S, Rezai MS, Ribeiro ALP, Roberts NLS, Robinson SR, Roever L, Ronfani L, Roshandel G, Rostami A, Roth GA, Roy A, Rubagotti E, Sachdev PS, Sadat N, Saddik B, Sadeghi E, Saeedi Moghaddam S, Safari H, Safari Y, Safari-Faramani R, Safdarian M, Safi S, Safiri S, Sagar R, Sahebkar A, Sahraian MA, Sajadi HS, Salam N, Salama JS, Salamati P, Saleem K, Saleem Z, Salimi Y, Salomon JA, Salvi SS, Salz I, Samy AM, Sanabria J, Sang Y, Santomauro DF, Santos IS, Santos JV, Santric Milicevic MM, Sao Jose BP, Sardana M, Sarker AR, Sarrafzadegan N, Sartorius B, Sarvi S, Sathian B, Satpathy M, Sawant AR, Sawhney M, Saxena S, Saylan M, Schaeffner E, Schmidt MI, Schneider IJC, Schöttker B, Schwebel DC, Schwendicke F, Scott JG, Sekerija M, Sepanlou SG, Serván-Mori E, Seyedmousavi S, Shabaninejad H, Shafieesabet A, Shahbazi M, Shaheen AA, Shaikh MA, ShamsBeyranvand M, Shamsi M, Shamsizadeh M, Sharafi H, Sharafi K, Sharif M, Sharif-Alhoseini M, Sharma M, Sharma R, She J, Sheikh A, Shi P, Shibuya K, Shigematsu M, Shiri R, Shirkoohi R, 
Shishani K, Shiue I, Shokraneh F, Shoman H, Shrime MG, Si S, Siabani S, Siddiqi TJ, Sigfusdottir ID, Sigurvinsdottir R, Silva JP, Silveira DGA, Singam NSV, Singh JA, Singh NP, Singh V, Sinha DN, Skiadaresi E, Slepak ELN, Sliwa K, Smith DL, Smith M, Soares Filho AM, Sobaih BH, Sobhani S, Sobngwi E, Soneji SS, Soofi M, Soosaraei M, Sorensen RJD, Soriano JB, Soyiri IN, Sposato LA, Sreeramareddy CT, Srinivasan V, Stanaway JD, Stein DJ, Steiner C, Steiner TJ, Stokes MA, Stovner LJ, Subart ML, Sudaryanto A, Sufiyan MB, Sunguya BF, Sur PJ, Sutradhar I, Sykes BL, Sylte DO, Tabarés-Seisdedos R, Tadakamadla SK, Tadesse BT, Tandon N, Tassew SG, Tavakkoli M, Taveira $\mathrm{N}$, Taylor HR, Tehrani-Banihashemi A, Tekalign TG, Tekelemedhin SW, Tekle MG, Temesgen H, Temsah M-H, Temsah O, Terkawi AS, Teweldemedhin M, Thankappan KR, Thomas N, Tilahun B, To QG, Tonelli M, Topor-Madry R, Topouzis F, Torre AE, Tortajada-Girbés M, Touvier M, TovaniPalone MR, Towbin JA, Tran BX, Tran KB, Troeger CE, Truelsen TC, Tsilimbaris MK, Tsoi D, Tudor Car L, Tuzcu EM, Ukwaja KN, Ullah I, Undurraga EA, Unutzer J, Updike RL, Usman MS, Uthman OA, Vaduganathan M, Vaezi A, Valdez PR, Varughese S, Vasankari TJ, Venketasubramanian N, Villafaina S, Violante FS, Vladimirov SK, Vlassov V, Vollset SE, Vosoughi K, Vujcic IS, Wagnew FS, Waheed Y, Waller SG, Wang Y, Wang Y-P, Weiderpass E, Weintraub RG, Weiss DJ, Weldegebreal F, Weldegwergs KG, Werdecker A, West TE, Whiteford HA, Widecka J, Wijeratne T, Wilner LB, Wilson S, Winkler AS, Wiyeh AB, Wiysonge CS, Wolfe CDA, Woolf AD, Wu S, Wu Y-C, Wyper GMA, Xavier D, Xu G, Yadgir S, Yadollahpour A, Yahyazadeh Jabbari SH, Yamada T, Yan LL, Yano Y, Yaseri M, Yasin YJ, Yeshaneh A, Yimer EM, Yip P, Yisma E, Yonemoto N, Yoon S-J, Yotebieng M, Younis MZ, Yousefifard M, Yu C, Zadnik V, Zaidi Z, Zaman SB, Zamani M, Zare Z, Zeleke AJ, Zenebe ZM, Zhang K, Zhao Z, Zhou M, Zodpey S, Zucker I, Vos T, Murray CJL (2018): Global, regional, and national incidence, prevalence, and years lived with disability for 354 diseases and injuries for 195 countries and territories, 1990-2017: a systematic analysis for the Global Burden of Disease Study 2017. Lancet 392:1789-1858.

http://www.sciencedirect.com/science/article/pii/S0140673618322797.

Jeurissen B, Leemans A, Tournier J-D, Jones DK, Sijbers J (2013): Investigating the prevalence of complex fiber configurations in white matter tissue with diffusion magnetic resonance imaging. Hum Brain Mapp 34:2747-2766. http://dx.doi.org/10.1002/hbm.22099.

Jogia J, Dima D, Frangou S (2012): Sex differences in bipolar disorder: a review of neuroimaging findings and new evidence. Bipolar Disord 14:461-471. http://dx.doi.org/10.1111/j.1399-5618.2012.01014.x.

Johannsen TH, Main KM, Ljubicic ML, Jensen TK, Andersen HR, Andersen MS, Petersen JH, Andersson A-M, Juul A (2018): Sex Differences in Reproductive Hormones During Mini-Puberty in Infants With Normal and Disordered Sex Development. J Clin Endocrinol Metab 103:3028-3037. http://dx.doi.org/10.1210/jc.2018-00482.

Johnson JK, Diehl J, Mendez MF, Neuhaus J, Shapira JS, Forman M, Chute DJ, Roberson ED, PaceSavitsky C, Neumann M, Chow TW, Rosen HJ, Forstl H, Kurz A, Miller BL (2005): Frontotemporal lobar degeneration: demographic characteristics of 353 patients. Arch Neurol 62:925-930. http://dx.doi.org/10.1001/archneur.62.6.925.

Jones EM, Okpala M, Zhang X, Parsha K, Keser Z, Kim CY, Wang A, Okpala N, Jagolino A, Savitz SI, Sharrief AZ (2020): Racial disparities in post-stroke functional outcomes in young patients with ischemic stroke. J Stroke Cerebrovasc Dis 29:104987. http://dx.doi.org/10.1016/j.jstrokecerebrovasdis.2020.104987.

Jones PB (2013): Adult mental health disorders and their age at onset. Br J Psychiatry Suppl 54:s5-10. http://dx.doi.org/10.1192/bjp.bp.112.119164.

Jones SAV, Vann Jones SA, O'Brien JT (2014): The prevalence and incidence of dementia with Lewy bodies: a systematic review of population and clinical studies. Psychological Medicine. http://dx.doi.org/10.1017/s0033291713000494.

de Jonge P, Roest AM, Lim CCW, Florescu SE, Bromet EJ, Stein DJ, Harris M, Nakov V, Caldas-deAlmeida JM, Levinson D, Al-Hamzawi AO, Haro JM, Viana MC, Borges G, O'Neill S, de Girolamo G, Demyttenaere K, Gureje O, Iwata N, Lee S, Hu C, Karam A, Moskalewicz J, Kovess-Masfety V, Navarro-Mateu F, Browne MO, Piazza M, Posada-Villa J, Torres Y, Ten Have ML, Kessler RC, Scott KM (2016): Cross-national epidemiology of panic disorder and panic attacks in the world mental health surveys. Depress Anxiety 33:1155-1177. http://dx.doi.org/10.1002/da.22572.

Kaczkurkin AN, Raznahan A, Satterthwaite TD (2019): Sex differences in the developing brain: insights from multimodal neuroimaging. Neuropsychopharmacology 44:71-85. http://dx.doi.org/10.1038/s41386-018-0111-z. 
Kalincik T, Vivek V, Jokubaitis V, Lechner-Scott J, Trojano M, Izquierdo G, Lugaresi A, Grand'maison F, Hupperts R, Oreja-Guevara C, Bergamaschi R, Iuliano G, Alroughani R, Van Pesch V, Amato MP, Slee M, Verheul F, Fernandez-Bolanos R, Fiol M, Spitaleri DL, Cristiano E, Gray O, Cabrera-Gomez JA, Shaygannejad V, Herbert J, Vucic S, Needham M, Petkovska-Boskova T, Sirbu C-A, Duquette P, Girard M, Grammond P, Boz C, Giuliani G, Rio ME, Barnett M, Flechter S, Moore F, Singhal B, Bacile EA, Saladino ML, Shaw C, Skromne E, Poehlau D, Vella N, Spelman T, Liew D, Kilpatrick TJ, Butzkueven H, MSBase Study Group (2013): Sex as a determinant of relapse incidence and progressive course of multiple sclerosis. Brain 136:3609-3617. http://dx.doi.org/10.1093/brain/awt281.

Kalkonde YV, Jawaid A, Qureshi SU, Shirani P, Wheaton M, Pinto-Patarroyo GP, Schulz PE (2012): Medical and environmental risk factors associated with frontotemporal dementia: a case-control study in a veteran population. Alzheimers Dement 8:204-210. http://dx.doi.org/10.1016/j.jalz.2011.03.011.

Kane JPM, Surendranathan A, Bentley A, Barker SAH, Taylor J-P, Thomas AJ, Allan LM, McNally RJ, James PW, McKeith IG, Burn DJ, O'Brien JT (2018): Clinical prevalence of Lewy body dementia. Alzheimers Res Ther 10:19. http://dx.doi.org/10.1186/s13195-018-0350-6.

Kang D-Z, Chen F-Y, Wang F-Y, Wu G-R, Liu Y, Wu G, Yu L-H, Lin Y-X, Lin Z-Y (2015): Brain gray matter volume changes associated with motor symptoms in patients with Parkinson's disease. Chinese Neurosurgical Journal 1:9. https://doi.org/10.1186/s41016-015-0003-6.

Kantarci K, Tosakulwong N, Lesnick TG, Zuk SM, Gunter JL, Gleason CE, Wharton W, Dowling NM, Vemuri P, Senjem ML, Shuster LT, Bailey KR, Rocca WA, Jack CR Jr, Asthana S, Miller VM (2016a): Effects of hormone therapy on brain structure: A randomized controlled trial. Neurology 87:887-896. http://dx.doi.org/10.1212/WNL.0000000000002970.

Kantarci OH (2019): Phases and Phenotypes of Multiple Sclerosis. Continuum 25:636-654. http://dx.doi.org/10.1212/CON.0000000000000737.

Kantarci OH, Lebrun C, Siva A, Keegan MB, Azevedo CJ, Inglese M, Tintoré M, Newton BD, DurandDubief F, Amato MP, De Stefano N, Sormani MP, Pelletier D, Okuda DT (2016b): Primary Progressive Multiple Sclerosis Evolving From Radiologically Isolated Syndrome. Annals of Neurology. http://dx.doi.org/10.1002/ana.24564.

Kao Y-H, Chou M-C, Chen C-H, Yang Y-H (2019): White Matter Changes in Patients with Alzheimer's Disease and Associated Factors. Journal of Clinical Medicine. http://dx.doi.org/10.3390/jcm8020167.

Kates R, Atkinson D, Brant-Zawadzki M (1996): Fluid-attenuated inversion recovery (FLAIR): clinical prospectus of current and future applications. Top Magn Reson Imaging 8:389-396. https://www.ncbi.nlm.nih.gov/pubmed/9402679.

Kelly S, Jahanshad N, Zalesky A, Kochunov P, Agartz I, Alloza C, Andreassen OA, Arango C, Banaj N, Bouix S, Bousman CA, Brouwer RM, Bruggemann J, Bustillo J, Cahn W, Calhoun V, Cannon D, Carr V, Catts S, Chen J, Chen J-X, Chen X, Chiapponi C, Cho KK, Ciullo V, Corvin AS, Crespo-Facorro B, Cropley V, De Rossi P, Diaz-Caneja CM, Dickie EW, Ehrlich S, Fan F-M, Faskowitz J, FatourosBergman H, Flyckt L, Ford JM, Fouche J-P, Fukunaga M, Gill M, Glahn DC, Gollub R, Goudzwaard ED, Guo H, Gur RE, Gur RC, Gurholt TP, Hashimoto R, Hatton SN, Henskens FA, Hibar DP, Hickie IB, Hong LE, Horacek J, Howells FM, Hulshoff Pol HE, Hyde CL, Isaev D, Jablensky A, Jansen PR, Janssen J, Jönsson EG, Jung LA, Kahn RS, Kikinis Z, Liu K, Klauser P, Knöchel C, Kubicki M, Lagopoulos J, Langen C, Lawrie S, Lenroot RK, Lim KO, Lopez-Jaramillo C, Lyall A, Magnotta V, Mandl RCW, Mathalon DH, McCarley RW, McCarthy-Jones S, McDonald C, McEwen S, McIntosh A, Melicher T, Mesholam-Gately RI, Michie PT, Mowry B, Mueller BA, Newell DT, O'Donnell P, OertelKnöchel V, Oestreich L, Paciga SA, Pantelis C, Pasternak O, Pearlson G, Pellicano GR, Pereira A, Pineda Zapata J, Piras F, Potkin SG, Preda A, Rasser PE, Roalf DR, Roiz R, Roos A, Rotenberg D, Satterthwaite TD, Savadjiev P, Schall U, Scott RJ, Seal ML, Seidman LJ, Shannon Weickert C, Whelan CD, Shenton ME, Kwon JS, Spalletta G, Spaniel F, Sprooten E, Stäblein M, Stein DJ, Sundram S, Tan Y, Tan S, Tang S, Temmingh HS, Westlye LT, Tønnesen S, Tordesillas-Gutierrez D, Doan NT, Vaidya J, van Haren NEM, Vargas CD, Vecchio D, Velakoulis D, Voineskos A, Voyvodic JQ, Wang Z, Wan P, Wei D, Weickert TW, Whalley H, White T, Whitford TJ, Wojcik JD, Xiang H, Xie Z, Yamamori H, Yang F, Yao N, Zhang G, Zhao J, van Erp TGM, Turner J, Thompson PM, Donohoe G (2018): Widespread white matter microstructural differences in schizophrenia across 4322 individuals: results from the ENIGMA Schizophrenia DTI Working Group. Mol Psychiatry 23:12611269. http://dx.doi.org/10.1038/mp.2017.170.

Kessler RC, Amminger GP, Aguilar-Gaxiola S, Alonso J, Lee S, Ustun TB (2007): Age of onset of mental 
disorders: a review of recent literature. Curr Opin Psychiatry 20:359.

https://www.ncbi.nlm.nih.gov/pmc/articles/pmc1925038/.

Kessler RC, Chiu WT, Demler O, Merikangas KR, Walters EE (2005): Prevalence, severity, and comorbidity of 12-month DSM-IV disorders in the National Comorbidity Survey Replication. Arch Gen Psychiatry 62:617-627. http://dx.doi.org/10.1001/archpsyc.62.6.617.

Kessler RC, Petukhova M, Sampson NA, Zaslavsky AM, Wittchen H-U (2012): Twelve-month and lifetime prevalence and lifetime morbid risk of anxiety and mood disorders in the United States. Int $\mathrm{J}$ Methods Psychiatr Res 21:169-184. https://onlinelibrary.wiley.com/doi/abs/10.1002/mpr.1359.

Keyes KM, Grant BF, Hasin DS (2008): Evidence for a closing gender gap in alcohol use, abuse, and dependence in the United States population. Drug Alcohol Depend 93:21-29. http://dx.doi.org/10.1016/j.drugalcdep.2007.08.017.

Kilpatrick DG, Resnick HS, Milanak ME, Miller MW, Keyes KM, Friedman MJ (2013): National estimates of exposure to traumatic events and PTSD prevalence using DSM-IV and DSM-5 criteria. J Trauma Stress 26:537-547. http://dx.doi.org/10.1002/jts.21848.

Kim JE, Song IH, Lee S-H (2017): Gender Differences of Stressful Life Events, Coping Style, Symptom Severity, and Health-Related Quality of Life in Patients With Panic Disorder. J Nerv Ment Dis 205:714-719. http://dx.doi.org/10.1097/NMD.0000000000000696.

Kim S, Kim MJ, Kim S, Kang HS, Lim SW, Myung W, Lee Y, Hong CH, Choi SH, Na DL, Seo SW, Ku BD, Kim SY, Kim SY, Jeong JH, Park SA, Carroll BJ, Kim DK (2015): Gender differences in risk factors for transition from mild cognitive impairment to Alzheimer's disease: A CREDOS study. Comprehensive Psychiatry. http://dx.doi.org/10.1016/j.comppsych.2015.07.002.

Klistorner A, Wang C, Yiannikas C, Parratt J, Dwyer M, Barton J, Graham SL, You Y, Liu S, Barnett MH (2018): Evidence of progressive tissue loss in the core of chronic MS lesions: A longitudinal DTI study. Neuroimage Clin 17:1028-1035. http://dx.doi.org/10.1016/j.nicl.2017.12.010.

Knopman DS, Roberts RO (2011): Estimating the number of persons with frontotemporal lobar degeneration in the US population. J Mol Neurosci 45:330-335. http://dx.doi.org/10.1007/s12031011-9538-y.

Kochunov P, Jahanshad N, Marcus D, Winkler A, Sprooten E, Nichols TE, Wright SN, Hong LE, Patel B, Behrens T, Jbabdi S, Andersson J, Lenglet C, Yacoub E, Moeller S, Auerbach E, Ugurbil K, Sotiropoulos SN, Brouwer RM, Landman B, Lemaitre H, den Braber A, Zwiers MP, Ritchie S, van Hulzen K, Almasy L, Curran J, deZubicaray GI, Duggirala R, Fox P, Martin NG, McMahon KL, Mitchell B, Olvera RL, Peterson C, Starr J, Sussmann J, Wardlaw J, Wright M, Boomsma DI, Kahn R, de Geus EJC, Williamson DE, Hariri A, van 't Ent D, Bastin ME, McIntosh A, Deary IJ, Hulshoff Pol HE, Blangero J, Thompson PM, Glahn DC, Van Essen DC (2015): Heritability of fractional anisotropy in human white matter: a comparison of Human Connectome Project and ENIGMA-DTI data. Neuroimage 111:300-311. http://dx.doi.org/10.1016/j.neuroimage.2015.02.050.

Koenen KC, Ratanatharathorn A, Ng L, McLaughlin KA, Bromet EJ, Stein DJ, Karam EG, Meron Ruscio A, Benjet C, Scott K, Atwoli L, Petukhova M, Lim CCW, Aguilar-Gaxiola S, Al-Hamzawi A, Alonso J, Bunting B, Ciutan M, de Girolamo G, Degenhardt L, Gureje O, Haro JM, Huang Y, Kawakami N, Lee S, Navarro-Mateu F, Pennell B-E, Piazza M, Sampson N, Ten Have M, Torres Y, Viana MC, Williams D, Xavier M, Kessler RC (2017): Posttraumatic stress disorder in the World Mental Health Surveys. Psychol Med 47:2260-2274. http://dx.doi.org/10.1017/S0033291717000708.

Kong L, Chen K, Womer F, Jiang W, Luo X, Driesen N, Liu J, Blumberg H, Tang Y, Xu K, Wang F (2013): Sex differences of gray matter morphology in cortico-limbic-striatal neural system in major depressive disorder. J Psychiatr Res 47:733-739. http://dx.doi.org/10.1016/j.jpsychires.2013.02.003.

Kong X-Z, Mathias SR, Guadalupe T, ENIGMA Laterality Working Group, Glahn DC, Franke B, Crivello F, Tzourio-Mazoyer N, Fisher SE, Thompson PM, Francks C (2018): Mapping cortical brain asymmetry in 17,141 healthy individuals worldwide via the ENIGMA Consortium. Proc Natl Acad Sci U S A 115:E5154-E5163. http://dx.doi.org/10.1073/pnas.1718418115.

Koran MEI, Wagener M, Hohman TJ, Alzheimer's Neuroimaging Initiative (2017): Sex differences in the association between AD biomarkers and cognitive decline. Brain Imaging Behav 11:205-213. http://dx.doi.org/10.1007/s11682-016-9523-8.

Kørner A, Lopez AG, Lauritzen L, Andersen PK, Kessing LV (2009): Late and very-late first-contact schizophrenia and the risk of dementia--a nationwide register based study. International Journal of Geriatric Psychiatry: A journal of the psychiatry of late life and allied sciences 24:61-67. https://onlinelibrary.wiley.com/doi/abs/10.1002/gps.2075?casa_token=SIZ65iaX5kUAAAAA:2- 
ysPmuZJ-4I3TIJ66vj_xrza98IAf0fLjCct6MYocCpzj_f3pWvs3kYaFXNureWSY6PNfhbvzK48VrW.

Koutsouleris N, Davatzikos C, Borgwardt S, Gaser C, Bottlender R, Frodl T, Falkai P, Riecher-Rössler A, Möller H-J, Reiser M, Pantelis C, Meisenzahl E (2014): Accelerated brain aging in schizophrenia and beyond: a neuroanatomical marker of psychiatric disorders. Schizophr Bull 40:1140-1153. http://dx.doi.org/10.1093/schbul/sbt142.

Kurian AK, Cardarelli KM (2007): Racial and ethnic differences in cardiovascular disease risk factors: a systematic review. Ethn Dis 17:143-152. https://www.ncbi.nlm.nih.gov/pubmed/17274224.

Kuring JK, Mathias JL, Ward L (2020): Risk of Dementia in persons who have previously experienced clinically-significant Depression, Anxiety, or PTSD: A Systematic Review and Meta-Analysis. J Affect Disord 274:247-261. http://dx.doi.org/10.1016/j.jad.2020.05.020.

Laansma MA, Bright JK, Al-Bachari S, Anderson TJ (2020): An International Multicenter Analysis of Brain Structure across Clinical Stages of Parkinson's Disease: The ENIGMA-Parkinson's Study. medRxiv. https://www.medrxiv.org/content/10.1101/2020.04.28.20072710v1.abstract.

Lai C-H (2019): Promising Neuroimaging Biomarkers in Depression. Psychiatry Investig 16:662-670. http://dx.doi.org/10.30773/pi.2019.07.25.2.

La Joie R, Perrotin A, Barré L, Hommet C, Mézenge F, Ibazizene M, Camus V, Abbas A, Landeau B, Guilloteau D, de La Sayette V, Eustache F, Desgranges B, Chételat G (2012): Region-specific hierarchy between atrophy, hypometabolism, and $\beta$-amyloid $(A \beta)$ load in Alzheimer's disease dementia. J Neurosci 32:16265-16273. http://dx.doi.org/10.1523/JNEUROSCI.2170-12.2012.

Lancaster SL, Melka SE, Rodriguez BF, Bryant AR (2014): PTSD Symptom Patterns Following Traumatic and Nontraumatic Events. J Aggress Maltreat Trauma 23:414-429. https://doi.org/10.1080/10926771.2014.893276.

de Lange A-MG, Barth C, Kaufmann T, Maximov II, van der Meer D, Agartz I, Westlye LT (2020): Women's brain aging: Effects of sex-hormone exposure, pregnancies, and genetic risk for Alzheimer's disease. Hum Brain Mapp 41:5141-5150. http://dx.doi.org/10.1002/hbm.25180.

Laskey P, Bates EA, Taylor JC (2019): A systematic literature review of intimate partner violence victimisation: An inclusive review across gender and sexuality. Aggress Violent Behav 47:1-11. http://www.sciencedirect.com/science/article/pii/S1359178918301095.

Lassmann H, Brück W, Lucchinetti CF (2007): The immunopathology of multiple sclerosis: an overview. Brain Pathol 17:210-218. http://dx.doi.org/10.1111/j.1750-3639.2007.00064.x.

Laws KR, Irvine K, Gale TM (2016): Sex differences in cognitive impairment in Alzheimer's disease. World J Psychiatry 6:54-65. http://dx.doi.org/10.5498/wjp.v6.i1.54.

Lee J, Cho H, Jeon S, Kim HJ, Kim YJ, Lee J, Kim ST, Lee J-M, Chin J, Lockhart SN, Lee AY, Na DL, Seo SW (2018): Sex-Related Reserve Hypothesis in Alzheimer's Disease: Changes in Cortical Thickness with a Five-Year Longitudinal Follow-Up. J Alzheimers Dis 65:641-649. http://dx.doi.org/10.3233/JAD-180049.

Lee S, Viqar F, Zimmerman ME, Narkhede A, Tosto G, Benzinger TLS, Marcus DS, Fagan AM, Goate A, Fox NC, Cairns NJ, Holtzman DM, Buckles V, Ghetti B, McDade E, Martins RN, Saykin AJ, Masters CL, Ringman JM, Ryan NS, Förster S, Laske C, Schofield PR, Sperling RA, Salloway S, Correia S, Jack C, Weiner M, Bateman RJ, Morris JC, Mayeux R, Brickman AM, for the Dominantly Inherited Alzheimer Network (2016): White matter hyperintensities are a core feature of Alzheimer's disease: Evidence from the dominantly inherited Alzheimer network. Annals of Neurology. http://dx.doi.org/10.1002/ana.24647.

Lee SK (2018): Sex as an important biological variable in biomedical research. BMB Rep 51:167-173. http://dx.doi.org/10.5483/bmbrep.2018.51.4.034.

Lehavot K, Katon JG, Chen JA, Fortney JC, Simpson TL (2018): Post-traumatic Stress Disorder by Gender and Veteran Status. Am J Prev Med 54:e1-e9. http://dx.doi.org/10.1016/j.amepre.2017.09.008.

Lemogne C, Delaveau P, Freton M, Guionnet S, Fossati P (2012): Medial prefrontal cortex and the self in major depression. J Affect Disord 136:e1-e11. http://dx.doi.org/10.1016/j.jad.2010.11.034.

Lenroot RK, Giedd JN (2010): Sex differences in the adolescent brain. Brain Cogn 72:46-55. http://dx.doi.org/10.1016/j.bandc.2009.10.008.

Lepore SJ, Revenson TA, Weinberger SL, Weston P, Frisina PG, Robertson R, Portillo MM, Jones H, Cross W (2006): Effects of social stressors on cardiovascular reactivity in Black and White women. Ann Behav Med 31:120-127. http://dx.doi.org/10.1207/s15324796abm3102_3.

Leuzy A, Chiotis K, Lemoine L, Gillberg P-G, Almkvist O, Rodriguez-Vieitez E, Nordberg A (2019): Tau 
PET imaging in neurodegenerative tauopathies-still a challenge. Mol Psychiatry 24:1112-1134. http://dx.doi.org/10.1038/s41380-018-0342-8.

LeWinn KZ, Sheridan MA, Keyes KM, Hamilton A, McLaughlin KA (2017): Sample composition alters associations between age and brain structure. Nat Commun 8:874. http://dx.doi.org/10.1038/s41467017-00908-7.

Lezak MD, Howieson DB, Bigler ED, Tranelet D (2004): Neuropsychological Assessment, 5th Edn Oxford: Oxford University Press.[Google Scholar].

Liao M, Yang F, Zhang Y, He Z, Su L, Li L (2014): Lack of gender effects on gray matter volumes in adolescent generalized anxiety disorder. J Affect Disord 155:278-282. http://dx.doi.org/10.1016/j.jad.2013.10.049.

Liesinger AM, Graff-Radford NR, Duara R, Carter RE, Hanna AI-Shaikh FS, Koga S, Hinkle KM, DiLello SK, Johnson MF, Aziz A, Ertekin-Taner N, Ross OA, Dickson DW, Murray ME (2018): Sex and age interact to determine clinicopathologic differences in Alzheimer's disease. Acta Neuropathol 136:873-885. http://dx.doi.org/10.1007/s00401-018-1908-x.

Lijster JM de, Dierckx B, Utens EMWJ, Verhulst FC, Zieldorff C, Dieleman GC, Legerstee JS (2017): The Age of Onset of Anxiety Disorders: A Meta-analysis. Can J Psychiatry 62:237-246. https://doi.org/10.1177/0706743716640757.

Lindamer LA, Lohr JB, Harris MJ, McAdams LA, Jeste DV (1999): Gender-related clinical differences in older patients with schizophrenia. J Clin Psychiatry 60:61-7; quiz 68-9. http://dx.doi.org/10.4088/jcp.v60n0114.

Li R, Ma X, Wang G, Yang J, Wang C (2016): Why sex differences in schizophrenia? J Transl Neurosci (Beijing) 1:37-42. https://www.ncbi.nlm.nih.gov/pubmed/29152382.

Lisofsky N, Riediger M, Gallinat J, Lindenberger U, Kühn S (2016): Hormonal contraceptive use is associated with neural and affective changes in healthy young women. Neuroimage 134:597-606. http://dx.doi.org/10.1016/j.neuroimage.2016.04.042.

Logue MW, van Rooij SJH, Dennis EL, Davis SL, Hayes JP, Stevens JS, Densmore M, Haswell CC, Ipser J, Koch SBJ, Korgaonkar M, Lebois LAM, Peverill M, Baker JT, Boedhoe PSW, Frijling JL, Gruber SA, Harpaz-Rotem I, Jahanshad N, Koopowitz S, Levy I, Nawijn L, O'Connor L, Olff M, Salat DH, Sheridan MA, Spielberg JM, van Zuiden M, Winternitz SR, Wolff JD, Wolf EJ, Wang X, Wrocklage K, Abdallah CG, Bryant RA, Geuze E, Jovanovic T, Kaufman ML, King AP, Krystal JH, Lagopoulos J, Bennett M, Lanius R, Liberzon I, McGlinchey RE, McLaughlin KA, Milberg WP, Miller MW, Ressler KJ, Veltman DJ, Stein DJ, Thomaes K, Thompson PM, Morey RA (2018): Smaller Hippocampal Volume in Posttraumatic Stress Disorder: A Multisite ENIGMA-PGC Study: Subcortical Volumetry Results From Posttraumatic Stress Disorder Consortia. Biol Psychiatry 83:244-253. http://www.sciencedirect.com/science/article/pii/S000632231731990X.

Longstreth WT Jr, Bernick C, Manolio TA, Bryan N, Jungreis CA, Price TR (1998): Lacunar infarcts defined by magnetic resonance imaging of 3660 elderly people: the Cardiovascular Health Study. Arch Neurol 55:1217-1225. http://dx.doi.org/10.1001/archneur.55.9.1217.

López-Zurbano S, González-Pinto A (2019): Gender Differences in Bipolar Disorder. In: Sáenz-Herrero, M, editor. Psychopathology in Women: Incorporating Gender Perspective into Descriptive Psychopathology. Cham: Springer International Publishing. pp 739-764. https://doi.org/10.1007/9783-030-15179-9_31.

Lord FM (1967): A paradox in the interpretation of group comparisons. Psychol Bull 68:304-305. http://dx.doi.org/10.1037/h0025105.

Luchetti S, Fransen NL, van Eden CG, Ramaglia V, Mason M, Huitinga I (2018): Progressive multiple sclerosis patients show substantial lesion activity that correlates with clinical disease severity and sex: a retrospective autopsy cohort analysis. Acta Neuropathologica. http://dx.doi.org/10.1007/s00401-018-1818-y.

Luders E, Toga AW, Thompson PM (2014): Why size matters: differences in brain volume account for apparent sex differences in callosal anatomy: the sexual dimorphism of the corpus callosum. Neuroimage 84:820-824. http://dx.doi.org/10.1016/j.neuroimage.2013.09.040.

Luders E, Narr KL, Thompson PM, Rex DE, Woods RP, Deluca H, Jancke L, Toga AW (2006): Gender effects on cortical thickness and the influence of scaling. Hum Brain Mapp 27:314-324. http://dx.doi.org/10.1002/hbm.20187.

Luders E, Narr KL, Thompson PM, Woods RP, Rex DE, Jancke L, Steinmetz H, Toga AW (2005): Mapping cortical gray matter in the young adult brain: effects of gender. Neuroimage 26:493-501. 
http://dx.doi.org/10.1016/j.neuroimage.2005.02.010.

Luo A, Jung J, Longley M, Rosoff DB, Charlet K, Muench C, Lee J, Hodgkinson CA, Goldman D, Horvath S, Kaminsky ZA, Lohoff FW (2020): Epigenetic aging is accelerated in alcohol use disorder and regulated by genetic variation in APOL2. Neuropsychopharmacology 45:327-336. http://dx.doi.org/10.1038/s41386-019-0500-y.

Lyon M, Welton T, Varda A, Maller JJ, Broadhouse K, Korgaonkar MS, Koslow SH, Williams LM, Gordon E, Rush AJ, Grieve SM (2019): Gender-specific structural abnormalities in major depressive disorder revealed by fixel-based analysis. Neuroimage Clin 21:101668. http://dx.doi.org/10.1016/j.nicl.2019.101668.

Maclin JMA, Wang T, Xiao S (2019): Biomarkers for the diagnosis of Alzheimer's disease, dementia Lewy body, frontotemporal dementia and vascular dementia. Gen Psychiatr 32:e100054. http://dx.doi.org/10.1136/gpsych-2019-100054.

Madonna D, Delvecchio G, Soares JC, Brambilla P (2019): Structural and functional neuroimaging studies in generalized anxiety disorder: a systematic review. Braz J Psychiatry 41:336-362. http://dx.doi.org/10.1590/1516-4446-2018-0108.

Maki PM, Dumas J (2009): Mechanisms of action of estrogen in the brain: insights from human neuroimaging and psychopharmacologic studies. Semin Reprod Med 27:250-259. http://dx.doi.org/10.1055/s-0029-1216278.

Malykhin NV, Huang Y, Hrybouski S, Olsen F (2017): Differential vulnerability of hippocampal subfields and anteroposterior hippocampal subregions in healthy cognitive aging. Neurobiol Aging 59:121134. http://dx.doi.org/10.1016/j.neurobiolaging.2017.08.001.

Mamlouk GM, Dorris DM, Barrett LR, Meitzen J (2020): Sex bias and omission in neuroscience research is influenced by research model and journal, but not reported NIH funding. Front Neuroendocrinol 57:100835. http://dx.doi.org/10.1016/j.yfrne.2020.100835.

Mamsen LS, Ernst EH, Borup R, Larsen A, Olesen RH, Ernst E, Anderson RA, Kristensen SG, Andersen CY (2017): Temporal expression pattern of genes during the period of sex differentiation in human embryonic gonads. Sci Rep 7:15961. http://dx.doi.org/10.1038/s41598-017-15931-3.

Marcus SM, Kerber KB, Rush AJ, Wisniewski SR, Nierenberg A, Balasubramani GK, Ritz L, Kornstein S, Young EA, Trivedi MH (2008): Sex differences in depression symptoms in treatment-seeking adults: confirmatory analyses from the Sequenced Treatment Alternatives to Relieve Depression study. Compr Psychiatry 49:238-246. http://dx.doi.org/10.1016/j.comppsych.2007.06.012.

Maron E, Nutt D (2017): Biological markers of generalized anxiety disorder. Dialogues Clin Neurosci 19:147-158. https://www.ncbi.nlm.nih.gov/pubmed/28867939.

Márquez F, Yassa MA (2019): Neuroimaging Biomarkers for Alzheimer's Disease. Mol Neurodegener 14:21. http://dx.doi.org/10.1186/s13024-019-0325-5.

Marras A, Fineberg N, Pallanti S (2016): Obsessive compulsive and related disorders: comparing DSM-5 and ICD-11. CNS Spectr 21:324-333. http://dx.doi.org/10.1017/S1092852916000110.

Marshall CR, Hardy CJD, Volkmer A, Russell LL, Bond RL, Fletcher PD, Clark CN, Mummery CJ, Schott JM, Rossor MN, Fox NC, Crutch SJ, Rohrer JD, Warren JD (2018): Primary progressive aphasia: a clinical approach. J Neurol 265:1474-1490. http://dx.doi.org/10.1007/s00415-018-8762-6.

Martinez-Martin P, Falup Pecurariu C, Odin P, van Hilten JJ, Antonini A, Rojo-Abuin JM, Borges V, Trenkwalder C, Aarsland D, Brooks DJ, Ray Chaudhuri K (2012): Gender-related differences in the burden of non-motor symptoms in Parkinson's disease. J Neurol 259:1639-1647. http://dx.doi.org/10.1007/s00415-011-6392-3.

Mason KA, Schoelwer MJ, Rogol AD (2020): Androgens During Infancy, Childhood, and Adolescence: Physiology and Use in Clinical Practice. Endocr Rev 41. http://dx.doi.org/10.1210/endrev/bnaa003.

Mathes BM, Morabito DM, Schmidt NB (2019): Epidemiological and Clinical Gender Differences in OCD. Curr Psychiatry Rep 21:36. http://dx.doi.org/10.1007/s11920-019-1015-2.

de Mathis MA, Diniz JB, Hounie AG, Shavitt RG, Fossaluza V, Ferrão Y, Leckman JF, de Bragança Pereira C, do Rosario MC, Miguel EC (2013): Trajectory in obsessive-compulsive disorder comorbidities. Eur Neuropsychopharmacol 23:594-601. http://dx.doi.org/10.1016/j.euroneuro.2012.08.006.

Maybin JA, Critchley HOD (2011): Progesterone: a pivotal hormone at menstruation. Ann N Y Acad Sci 1221:88-97. http://dx.doi.org/10.1111/j.1749-6632.2011.05953.x.

Mayeda ER (2019): Invited Commentary: Examining Sex/Gender Differences in Risk of Alzheimer Disease and Related Dementias-Challenges and Future Directions. Am J Epidemiol 188:1224- 
1227. https://academic.oup.com/aje/article-pdf/188/7/1224/28890427/kwz047.pdf.

Mayo AM, Peavy GM (2019): Associations among Braak stage, Parkinsonian gait, cognition, and functional status in autopsy-confirmed dementia with Lewy bodies. Int J Geriatr Psychiatry 34:738744. http://dx.doi.org/10.1002/gps.5080.

McCabe SE, West BT, Jutkiewicz EM, Boyd CJ (2017): Multiple DSM-5 substance use disorders: A national study of US adults. Human Psychopharmacology: Clinical and Experimental 32:e2625. https://onlinelibrary.wiley.com/doi/abs/10.1002/hup.2625?casa_token=fRDgrxHBolOAAAAA:WWQjtK gbhhBy5wT8MH5qM8KBgY7ELSWJJcgjqatfIC1c8C8KOWCXBdShXAUqEhfzKsLVVXxUlyHLLw.

McCarthy MM (2008): Estradiol and the developing brain. Physiol Rev 88:91-124. http://dx.doi.org/10.1152/physrev.00010.2007.

McCarthy MM, Arnold AP, Ball GF, Blaustein JD, De Vries GJ (2012): Sex differences in the brain: the not so inconvenient truth. J Neurosci 32:2241-2247. http://dx.doi.org/10.1523/JNEUROSCI.537211.2012.

McClellan ML, Reed BG, Becker JB (2017): Worlds Colliding: Trans-disciplinary approaches to gender and addictions. The Social History of Alcohol and Drugs. http://dx.doi.org/10.1086/shad31010107.

McGrath J, Saha S, Welham J, El Saadi O, MacCauley C, Chant D (2004): A systematic review of the incidence of schizophrenia: the distribution of rates and the influence of sex, urbanicity, migrant status and methodology. BMC Med 2:13. http://dx.doi.org/10.1186/1741-7015-2-13.

McHugh RK, Geyer RB, Chase AR, Griffin ML, Bogunovic O, Weiss RD (2021): Sex differences in benzodiazepine misuse among adults with substance use disorders. Addict Behav 112:106608. http://dx.doi.org/10.1016/j.addbeh.2020.106608.

McHugh RK, Votaw VR, Sugarman DE, Greenfield SF (2018): Sex and gender differences in substance use disorders. Clin Psychol Rev 66:12-23. http://dx.doi.org/10.1016/j.cpr.2017.10.012.

McKeith IG, Boeve BF, Dickson DW, Halliday G, Taylor J-P, Weintraub D, Aarsland D, Galvin J, Attems J, Ballard CG, Bayston A, Beach TG, Blanc F, Bohnen N, Bonanni L, Bras J, Brundin P, Burn D, ChenPlotkin A, Duda JE, El-Agnaf O, Feldman H, Ferman TJ, Ffytche D, Fujishiro H, Galasko D, Goldman JG, Gomperts SN, Graff-Radford NR, Honig LS, Iranzo A, Kantarci K, Kaufer D, Kukull W, Lee VMY, Leverenz JB, Lewis S, Lippa C, Lunde A, Masellis M, Masliah E, McLean P, Mollenhauer B, Montine TJ, Moreno E, Mori E, Murray M, O'Brien JT, Orimo S, Postuma RB, Ramaswamy S, Ross OA, Salmon DP, Singleton A, Taylor A, Thomas A, Tiraboschi P, Toledo JB, Trojanowski JQ, Tsuang D, Walker Z, Yamada M, Kosaka K (2017): Diagnosis and management of dementia with Lewy bodies: Fourth consensus report of the DLB Consortium. Neurology 89:88-100. http://dx.doi.org/10.1212/WNL.0000000000004058.

McLean CP, Asnaani A, Litz BT, Hofmann SG (2011): Gender differences in anxiety disorders: prevalence, course of illness, comorbidity and burden of illness. J Psychiatr Res 45:1027-1035. http://dx.doi.org/10.1016/j.jpsychires.2011.03.006.

Mesulam MM, Weintraub S (1992): Spectrum of primary progressive aphasia. Baillieres Clin Neurol 1:583-609. https://www.ncbi.nlm.nih.gov/pubmed/1344204.

Mielke MM, Vemuri P, Rocca WA (2014): Clinical epidemiology of Alzheimer's disease: assessing sex and gender differences. Clin Epidemiol 6:37. https://www.ncbi.nlm.nih.gov/pmc/articles/pmc3891487/.

Mikkola TS, Savolainen-Peltonen H, Tuomikoski P, Hoti F, Vattulainen P, Gissler M, Ylikorkala O (2017): Lower Death Risk for Vascular Dementia Than for Alzheimer's Disease With Postmenopausal Hormone Therapy Users. J Clin Endocrinol Metab 102:870-877. http://dx.doi.org/10.1210/jc.20163590.

Miller DH, Chard DT, Ciccarelli O (2012): Clinically isolated syndromes. Lancet Neurol 11:157-169. http://dx.doi.org/10.1016/S1474-4422(11)70274-5.

Miller GA, Chapman JP (2001): Misunderstanding analysis of covariance. J Abnorm Psychol 110:40-48. http://dx.doi.org/10.1037//0021-843x.110.1.40.

Monnig MA, Yeo RA, Tonigan JS, McCrady BS, Thoma RJ, Sabbineni A, Hutchison KE (2015): Associations of White Matter Microstructure with Clinical and Demographic Characteristics in Heavy Drinkers. PLoS One 10:e0142042. http://dx.doi.org/10.1371/journal.pone.0142042.

Montoya ER, Bos PA (2017): How Oral Contraceptives Impact Social-Emotional Behavior and Brain Function. Trends Cogn Sci 21:125-136. http://dx.doi.org/10.1016/j.tics.2016.11.005.

Moraga-Amaro R, Van Waarde A, Doorduin J, De Vries EFJ (2018): Sex steroid hormones and brain function: PET imaging as a tool for research. J Neuroendocrinol 30:e12565. 
https://onlinelibrary.wiley.com/doi/abs/10.1111/jne.12565.

Morris JC, Schindler SE, McCue LM, Moulder KL, Benzinger TLS, Cruchaga C, Fagan AM, Grant E, Gordon BA, Holtzman DM, Xiong C (2019): Assessment of Racial Disparities in Biomarkers for Alzheimer Disease. JAMA Neurol 76:264-273. http://dx.doi.org/10.1001/jamaneurol.2018.4249.

Mouton A, Blanc F, Gros A, Manera V, Fabre R, Sauleau E, Gomez-Luporsi I, Tifratene K, Friedman L, Thümmler S, Pradier C, Robert PH, David R (2018): Sex ratio in dementia with Lewy bodies balanced between Alzheimer's disease and Parkinson's disease dementia: a cross-sectional study. Alzheimers Res Ther 10:92. http://dx.doi.org/10.1186/s13195-018-0417-4.

Mrabet Khiari H, Achouri A, Ben Ali N, Cherif A, Batti H, Messaoud T, Mrabet A (2011): Obsessivecompulsive disorder: a new risk factor for Alzheimer disease? Neurol Sci 32:959-962. http://dx.doi.org/10.1007/s10072-011-0480-0.

Nabulsi L, Lawrence KE (2020): Exogenous sex hormone effects on brain microstructure in women: a diffusion MRI study in the UK Biobank. 16th International.

https://www.spiedigitallibrary.org/conference-proceedings-of-spie/11583/1158308/Exogenous-sexhormone-effects-on-brain-microstructure-inwomen/10.1117/12.2579631.short?casa_token=wCBIFZIrpfkAAAAA:UHckcFPoy25UE_4oa8IbLOC_ C7sp1D2qV_TBqmpCWJvXkxEbxl7VN2UYxQ_P1TkQUXZZdwdEdSU.

Narr KL, Thompson PM, Sharma T, Moussai J, Blanton R, Anvar B, Edris A, Krupp R, Rayman J, Khaledy M, Toga AW (2001): Three-dimensional mapping of temporo-limbic regions and the lateral ventricles in schizophrenia: gender effects. Biol Psychiatry 50:84-97. http://www.sciencedirect.com/science/article/pii/S0006322300011203.

Nasrabady SE, Rizvi B, Goldman JE, Brickman AM (2018): White matter changes in Alzheimer's disease: a focus on myelin and oligodendrocytes. Acta Neuropathologica Communications. http://dx.doi.org/10.1186/s40478-018-0515-3.

National Institute of Mental Health (2016): Schizophrenia. National Institute of Mental Health. https://www.nimh.nih.gov/health/topics/schizophrenia/index.shtml.

National Institute of Mental Health (2019): Post-Traumatic Stress Disorder. National Institute of Mental Health. https://www.nimh.nih.gov/health/topics/post-traumatic-stress-disorder-ptsd/index.shtml.

Negash A, Alem A, Kebede D, Deyessa N, Shibre T, Kullgren G (2005): Prevalence and clinical characteristics of bipolar I disorder in Butajira, Ethiopia: a community-based study. J Affect Disord 87:193-201. http://dx.doi.org/10.1016/j.jad.2005.03.011.

Neu SC, Pa J, Kukull W, Beekly D, Kuzma A, Gangadharan P, Wang L-S, Romero K, Arneric SP, Redolfi A, Orlandi D, Frisoni GB, Au R, Devine S, Auerbach S, Espinosa A, Boada M, Ruiz A, Johnson SC, Koscik R, Wang J-J, Hsu W-C, Chen Y-L, Toga AW (2017): Apolipoprotein E Genotype and Sex Risk Factors for Alzheimer Disease: A Meta-analysis. JAMA Neurol 74:1178-1189. http://dx.doi.org/10.1001/jamaneurol.2017.2188.

NIMH (2019): Obsessive-Compulsive Disorder. National Institute of Mental Health. https://www.nimh.nih.gov/health/topics/obsessive-compulsive-disorder-ocd/index.shtml.

Nisar S, Bhat AA, Hashem S, Syed N, Yadav SK, Uddin S, Fakhro K, Bagga P, Thompson P, Reddy R, Frenneaux MP, Haris M (2020): Genetic and Neuroimaging Approaches to Understanding PostTraumatic Stress Disorder. Int J Mol Sci 21. http://dx.doi.org/10.3390/ijms21124503.

Niu L, Matsui M, Zhou S-Y, Hagino H, Takahashi T, Yoneyama E, Kawasaki Y, Suzuki M, Seto H, Ono T, Kurachi M (2004): Volume reduction of the amygdala in patients with schizophrenia: a magnetic resonance imaging study. Psychiatry Res 132:41-51. http://dx.doi.org/10.1016/j.pscychresns.2004.06.002.

Nobis L, Manohar SG, Smith SM, Alfaro-Almagro F, Jenkinson M, Mackay CE, Husain M (2019): Hippocampal volume across age: Nomograms derived from over 19,700 people in UK Biobank. Neuroimage Clin 23:101904. http://dx.doi.org/10.1016/j.nicl.2019.101904.

Noh Y, Jeon S, Lee JM, Seo SW, Kim GH, Cho H, Ye BS, Yoon CW, Kim HJ, Chin J, Park KH, Heilman $\mathrm{KM}, \mathrm{Na}$ DL (2014): Anatomical heterogeneity of Alzheimer disease: based on cortical thickness on MRIs. Neurology 83:1936-1944. http://dx.doi.org/10.1212/WNL.0000000000001003.

Nugent BM, Tobet SA, Lara HE, Lucion AB, Wilson ME, Recabarren SE, Paredes AH (2012): Hormonal programming across the lifespan. Horm Metab Res 44:577-586. http://dx.doi.org/10.1055/s-00321312593

Nuru-Jeter AM, Michaels EK, Thomas MD, Reeves AN, Thorpe RJ Jr, LaVeist TA (2018): Relative Roles of Race Versus Socioeconomic Position in Studies of Health Inequalities: A Matter of Interpretation. 
Annu Rev Public Health 39:169-188. http://dx.doi.org/10.1146/annurev-publhealth-040617-014230.

O'Brien LM, Ziegler DA, Deutsch CK, Kennedy DN, Goldstein JM, Seidman LJ, Hodge S, Makris N,

Caviness V, Frazier JA, Herbert MR (2006): Adjustment for whole brain and cranial size in volumetric brain studies: a review of common adjustment factors and statistical methods. Harv Rev Psychiatry 14:141-151. http://dx.doi.org/10.1080/10673220600784119.

O'Dwyer L, Lamberton F, Bokde ALW, Ewers M, Faluyi YO, Tanner C, Mazoyer B, O'Neill D, Bartley M, Collins R, Coughlan T, Prvulovic D, Hampel H (2012): Sexual dimorphism in healthy aging and mild cognitive impairment: a DTI study. PLoS One 7:e37021.

http://dx.doi.org/10.1371/journal.pone.0037021.

Oettel M, Mukhopadhyay AK (2004): Progesterone: the forgotten hormone in men? Aging Male 7:236257. http://dx.doi.org/10.1080/13685530400004199.

Onyike CU, Diehl-Schmid J (2013): The epidemiology of frontotemporal dementia. Int Rev Psychiatry 25:130-137. http://dx.doi.org/10.3109/09540261.2013.776523.

Oppedal K, Ferreira D, Cavallin L, Lemstra AW, Ten Kate M, Padovani A, Rektorova I, Bonanni L, Wahlund L-O, Engedal K, Nobili F, Kramberger M, Taylor J-P, Hort J, Snædal J, Blanc F, Walker Z, Antonini A, Westman E, Aarsland D, Alzheimer's Disease Neuroimaging Initiative (2019): A signature pattern of cortical atrophy in dementia with Lewy bodies: A study on 333 patients from the European DLB consortium. Alzheimers Dement 15:400-409. http://dx.doi.org/10.1016/j.jalz.2018.09.011.

Ouellette R, Mangeat G, Polyak I, Warntjes M, Forslin Y, Bergendal Å, Plattén M, Uppman M, Treaba CA, Cohen-Adad J, Piehl F, Kristoffersen Wiberg M, Fredrikson S, Mainero C, Granberg T (2020): Validation of Rapid Magnetic Resonance Myelin Imaging in Multiple Sclerosis. Ann Neurol 87:710724. http://dx.doi.org/10.1002/ana.25705.

Outeiro TF, Koss DJ, Erskine D, Walker L, Kurzawa-Akanbi M, Burn D, Donaghy P, Morris C, Taylor J-P, Thomas A, Attems J, McKeith I (2019): Dementia with Lewy bodies: an update and outlook. Mol Neurodegener 14:5. http://dx.doi.org/10.1186/s13024-019-0306-8.

Pakkenberg H, Voigt J (1964): BRAIN WEIGHT OF THE DANES. Cells Tissues Organs 56:297-307. https://www.karger.com/DOI/10.1159/000142510.

Palk A, Illes J, Thompson PM, Stein DJ (2020): Ethical issues in global neuroimaging genetics collaborations. Neuroimage 221:117208. http://dx.doi.org/10.1016/j.neuroimage.2020.117208.

Panizzon MS, Hauger RL, Xian H, Jacobson K, Lyons MJ, Franz CE, Kremen WS (2018): Interactive effects of testosterone and cortisol on hippocampal volume and episodic memory in middle-aged men. Psychoneuroendocrinology 91:115-122. http://dx.doi.org/10.1016/j.psyneuen.2018.03.003.

Pasternak O, Sochen N, Gur Y, Intrator N, Assaf Y (2009): Free water elimination and mapping from diffusion MRI. Magn Reson Med 62:717-730. http://dx.doi.org/10.1002/mrm.22055.

Paul RH, Haque O, Gunstad J, Tate DF, Grieve SM, Hoth K, Brickman AM, Cohen R, Lange K, Jefferson AL, MacGregor KL, Gordon E (2005): Subcortical hyperintensities impact cognitive function among a select subset of healthy elderly. Arch Clin Neuropsychol 20:697-704. http://dx.doi.org/10.1016/j.acn.2005.02.004.

Paul R, Salminen L (2019): Vascular Cognitive Impairment. In: Ravdin, LD, Katzen, HL, editors. Handbook on the Neuropsychology of Aging and Dementia. Cham: Springer International Publishing. pp 479-488. https://doi.org/10.1007/978-3-319-93497-6_30.

Piguet O, Hornberger M, Mioshi E, Hodges JR (2011): Behavioural-variant frontotemporal dementia: diagnosis, clinical staging, and management. Lancet Neurol 10:162-172. http://dx.doi.org/10.1016/S1474-4422(10)70299-4.

Pike CJ (2017): Sex and the development of Alzheimer's disease. J Neurosci Res 95:671-680. http://dx.doi.org/10.1002/jnr.23827.

Pintzka CWS, Håberg AK (2015): Perimenopausal hormone therapy is associated with regional sparing of the CA1 subfield: a HUNT MRI study. Neurobiol Aging. https://www.sciencedirect.com/science/article/pii/S0197458015003073.

Piras F, Piras F, Abe Y, Agarwal SM, Anticevic A, Ameis S, Arnold P, Bargalló N, Batistuzzo MC, Benedetti F, Beucke J-C, Boedhoe PSW, Bollettini I, Brem S, Calvo A, Cho KIK, Dallaspezia S, Dickie E, Ely BA, Fan S, Fouche J-P, Gruner P, Gürsel DA, Hauser T, Hirano Y, Hoexter MQ, lorio M, James A, Reddy J, Kaufmann C, Koch K, Kochunov P, Kwon JS, Lazaro L, Lochner C, Marsh R, Nakagawa A, Nakamae T, Narayanaswamy JC, Sakai Y, Shimizu E, Simon D, Simpson HB, Soreni N, Stämpfli P, Stern ER, Szeszko P, Takahashi J, Venkatasubramanian G, Wang Z, Yun J-Y, ENIGMA OCD Working Group, Stein DJ, Jahanshad N, Thompson PM, van den Heuvel OA, 
Spalletta G (2019): White Matter Microstructure and its Relation to Clinical Features of ObsessiveCompulsive Disorder: Findings from the ENIGMA OCD Working Group. bioRxiv. https://www.biorxiv.org/content/10.1101/855916v1.full-text.

Piras F, Piras F, Chiapponi C, Girardi P, Caltagirone C, Spalletta G (2015): Widespread structural brain changes in OCD: a systematic review of voxel-based morphometry studies. Cortex 62:89-108. http://dx.doi.org/10.1016/j.cortex.2013.01.016.

du Plessis S, Scheffler F, Luckhoff H, Asmal L, Kilian S, Phahladira L, Emsley R (2020): Childhood trauma and hippocampal subfield volumes in first-episode schizophrenia and healthy controls. Schizophr Res. http://dx.doi.org/10.1016/j.schres.2019.10.009.

Podcasy JL, Epperson CN (2016): Considering sex and gender in Alzheimer disease and other dementias. Dialogues Clin Neurosci 18:437-446. https://www.ncbi.nlm.nih.gov/pubmed/28179815.

Prabhushankar R, Krueger C, Manrique C (2014): Membrane estrogen receptors: their role in blood pressure regulation and cardiovascular disease. Curr Hypertens Rep 16:408. http://dx.doi.org/10.1007/s11906-013-0408-6.

Prendergast BJ, Onishi KG, Zucker I (2014): Female mice liberated for inclusion in neuroscience and biomedical research. Neurosci Biobehav Rev 40:1-5. http://dx.doi.org/10.1016/j.neubiorev.2014.01.001.

Prince MJ, Acosta D, Guerra M, Huang Y, Jimenez-Velazquez IZ, Llibre Rodriguez JJ, Salas A, Sosa AL, Chua K-C, Dewey ME, Liu Z, Mayston R, Valhuerdi A (2018): Reproductive period, endogenous estrogen exposure and dementia incidence among women in Latin America and China; A 10/66 population-based cohort study. PLOS ONE. http://dx.doi.org/10.1371/journal.pone.0192889.

Prince MJ (2015): World Alzheimer Report 2015: the global impact of dementia: an analysis of prevalence, incidence, cost and trends.

Protopopescu X, Pan H, Tuescher O, Cloitre M, Goldstein M, Engelien A, Yang Y, Gorman J, LeDoux J, Stern E, Silbersweig D (2006): Increased brainstem volume in panic disorder: a voxel-based morphometric study. Neuroreport 17:361-363. http://dx.doi.org/10.1097/01.wnr.0000203354.80438.1.

Raber J, Huang Y, Ashford JW (2004): ApoE genotype accounts for the vast majority of AD risk and AD pathology. Neurobiol Aging 25:641-650. http://dx.doi.org/10.1016/j.neurobiolaging.2003.12.023.

Rabin RA, Mackey S, Parvaz MA, Cousijn J, Li C-S, Pearlson G, Schmaal L, Sinha R, Stein E, Veltman D, Others (2020): Common and gender-specific associations with cocaine use on gray matter volume: Data from the ENIGMA addiction working group. Hum Brain Mapp. https://onlinelibrary.wiley.com/doi/abs/10.1002/hbm.25141.

Raffelt DA, Smith RE, Ridgway GR, Tournier J-D, Vaughan DN, Rose S, Henderson R, Connelly A (2015): Connectivity-based fixel enhancement: Whole-brain statistical analysis of diffusion MRI measures in the presence of crossing fibres. Neuroimage 117:40-55. http://dx.doi.org/10.1016/j.neuroimage.2015.05.039.

Raffelt DA, Tournier J-D, Smith RE, Vaughan DN, Jackson G, Ridgway GR, Connelly A (2017): Investigating white matter fibre density and morphology using fixel-based analysis. Neuroimage 144:58-73. http://dx.doi.org/10.1016/j.neuroimage.2016.09.029.

Rakesh D, Allen NB, Whittle S (2020): Balancing act: Neural correlates of affect dysregulation in youth depression and substance use - A systematic review of functional neuroimaging studies. Dev Cogn Neurosci 42:100775. http://www.sciencedirect.com/science/article/pii/S1878929320300232.

Ramirez FD, Motazedian P, Jung RG, Di Santo P, MacDonald Z, Simard T, Clancy AA, Russo JJ, Welch V, Wells GA, Hibbert B (2017): Sex Bias Is Increasingly Prevalent in Preclinical Cardiovascular Research: Implications for Translational Medicine and Health Equity for Women: A Systematic Assessment of Leading Cardiovascular Journals Over a 10-Year Period. Circulation 135:625-626. http://dx.doi.org/10.1161/CIRCULATIONAHA.116.026668.

Ramirez-Gomez L, Zheng L, Reed B, Kramer J, Mungas D, Zarow C, Vinters H, Ringman JM, Chui H (2017): Neuropsychological Profiles Differentiate Alzheimer Disease from Subcortical Ischemic Vascular Dementia in an Autopsy-Defined Cohort. Dementia and Geriatric Cognitive Disorders. http://dx.doi.org/10.1159/000477344.

Ranasinghe KG, Rankin KP, Lobach IV, Kramer JH, Sturm VE, Bettcher BM, Possin K, Christine You S, Lamarre AK, Shany-Ur T, Stephens ML, Perry DC, Lee SE, Miller ZA, Gorno-Tempini ML, Rosen HJ, Boxer A, Seeley WW, Rabinovici GD, Vossel KA, Miller BL (2016): Cognition and neuropsychiatry in behavioral variant frontotemporal dementia by disease stage. Neurology 86:600-610. 
http://dx.doi.org/10.1212/WNL.0000000000002373.

Rando K, Tuit K, Hannestad J, Guarnaccia J, Sinha R (2013): Sex differences in decreased limbic and cortical grey matter volume in cocaine dependence: a voxel-based morphometric study. Addict Biol 18:147-160. http://dx.doi.org/10.1111/adb.12008.

Rasquin SMC, Verhey FRJ, Lousberg R, Winkens I, Lodder J (2002): Vascular cognitive disorders: memory, mental speed and cognitive flexibility after stroke. J Neurol Sci 203-204:115-119. http://dx.doi.org/10.1016/s0022-510x(02)00264-2.

Reynolds R, Roncaroli F, Nicholas R, Radotra B, Gveric D, Howell O (2011): The neuropathological basis of clinical progression in multiple sclerosis. Acta Neuropathol 122:155-170. http://dx.doi.org/10.1007/s00401-011-0840-0.

Ribe AR, Laursen TM, Charles M, Katon W, Fenger-Grøn M, Davydow D, Chwastiak L, Cerimele JM, Vestergaard M (2015): Long-term Risk of Dementia in Persons With Schizophrenia: A Danish Population-Based Cohort Study. JAMA Psychiatry 72:1095-1101. http://dx.doi.org/10.1001/jamapsychiatry.2015.1546.

Rintala H, Chudal R, Leppämäki S, Leivonen S, Hinkka-Yli-Salomäki S, Sourander A (2017): Registerbased study of the incidence, comorbidities and demographics of obsessive-compulsive disorder in specialist healthcare. BMC Psychiatry 17:64. http://dx.doi.org/10.1186/s12888-017-1224-3.

Ritchie H, Roser M (04/2018): Mental Health. Our World In Data. https://ourworldindata.org/mental-health.

Ritchie SJ, Cox SR, Shen X, Lombardo MV, Reus LM, Alloza C, Harris MA, Alderson HL, Hunter S, Neilson E, Liewald DCM, Auyeung B, Whalley HC, Lawrie SM, Gale CR, Bastin ME, Mclntosh AM, Deary IJ (2018): Sex Differences in the Adult Human Brain: Evidence from 5216 UK Biobank Participants. Cereb Cortex 28:2959-2975. http://dx.doi.org/10.1093/cercor/bhy109.

Rogalski E, Rademaker A, Weintraub S (2007): Primary progressive aphasia: relationship between gender and severity of language impairment. Cogn Behav Neurol 20:38-43. http://dx.doi.org/10.1097/WNN.0b013e31802e3bae.

Rohrer JD (2012): Structural brain imaging in frontotemporal dementia. Biochim Biophys Acta 1822:325332. http://dx.doi.org/10.1016/j.bbadis.2011.07.014.

Rojas JI, Patrucco L, Besada C, Funes J, Cristiano E (2013): Sex-related differences in atrophy and lesion load in multiple sclerosis patients. Neurología (English Edition) 28:389-393. http://www.sciencedirect.com/science/article/pii/S2173580813001211.

Rossetti MG, Mackey S, Patalay P, Batalla A, Bellani M, Chye YY, Conrod P, Cousijn J, Garavan H, Solowij N, Suo C, Thompson PM, Yucel M, Brambilla P, Lorenzetti V (2019): The neuroanatomy of cannabis use: does gender matter? Findings from the ENIGMA addiction working group. Eur Neuropsychopharmacol 29:S182-S183. http://www.sciencedirect.com/science/article/pii/S0924977X18311556.

Rosso SM, Landweer E-J, Houterman M, Donker Kaat L, van Duijn CM, van Swieten JC (2003): Medical and environmental risk factors for sporadic frontotemporal dementia: a retrospective case-control study. J Neurol Neurosurg Psychiatry 74:1574-1576. http://dx.doi.org/10.1136/jnnp.74.11.1574.

Rotgers E, Jørgensen A, Yao HH-C (2018): At the Crossroads of Fate-Somatic Cell Lineage Specification in the Fetal Gonad. Endocr Rev 39:739-759. http://dx.doi.org/10.1210/er.2018-00010.

Rothman MS, Carlson NE, Xu M, Wang C, Swerdloff R, Lee P, Goh VHH, Ridgway EC, Wierman ME (2011): Reexamination of testosterone, dihydrotestosterone, estradiol and estrone levels across the menstrual cycle and in postmenopausal women measured by liquid chromatography-tandem mass spectrometry. Steroids 76:177-182. http://dx.doi.org/10.1016/j.steroids.2010.10.010.

Rubinow DR, Schmidt PJ (2019): Sex differences and the neurobiology of affective disorders. Neuropsychopharmacology 44:111-128. http://dx.doi.org/10.1038/s41386-018-0148-z.

Ruigrok ANV, Salimi-Khorshidi G, Lai M-C, Baron-Cohen S, Lombardo MV, Tait RJ, Suckling J (2014): A meta-analysis of sex differences in human brain structure. Neurosci Biobehav Rev 39:34-50. http://dx.doi.org/10.1016/j.neubiorev.2013.12.004.

Ruscio AM, Stein DJ, Chiu WT, Kessler RC (2010): The epidemiology of obsessive-compulsive disorder in the National Comorbidity Survey Replication. Mol Psychiatry 15:53-63. http://dx.doi.org/10.1038/mp.2008.94.

Ruscio AM, Hallion LS, Lim CCW, Aguilar-Gaxiola S, Al-Hamzawi A, Alonso J, Andrade LH, Borges G, Bromet EJ, Bunting B, Caldas de Almeida JM, Demyttenaere K, Florescu S, de Girolamo G, Gureje O, Haro JM, He Y, Hinkov H, Hu C, de Jonge P, Karam EG, Lee S, Lepine J-P, Levinson D, Mneimneh Z, Navarro-Mateu F, Posada-Villa J, Slade T, Stein DJ, Torres Y, Uda H, Wojtyniak B, 
Kessler RC, Chatterji S, Scott KM (2017): Cross-sectional Comparison of the Epidemiology of DSM-5 Generalized Anxiety Disorder Across the Globe. JAMA Psychiatry 74:465-475.

http://dx.doi.org/10.1001/jamapsychiatry.2017.0056.

Salat DH, Greve DN, Pacheco JL, Quinn BT (2009): Regional white matter volume differences in nondemented aging and Alzheimer's disease. Neuroimage. https://www.sciencedirect.com/science/article/pii/S1053811908011245.

Salles A, Awad M, Goldin L, Krus K, Lee JV, Schwabe MT, Lai CK (2019): Estimating Implicit and Explicit Gender Bias Among Health Care Professionals and Surgeons. JAMA Netw Open 2:e196545. http://dx.doi.org/10.1001/jamanetworkopen.2019.6545.

Salminen LE, Conturo TE, Bolzenius JD, Cabeen RP, Akbudak E, Paul RH (2016): Reducing CSF partial volume effects to enhance diffusion tensor imaging metrics of brain microstructure. Technol Innov 18:5-20. http://dx.doi.org/10.21300/18.1.2016.5.

Sanchis-Segura C, Cruz-Gómez AJ, Belenguer A, Fittipaldi Márquez MS, Ávila C, Forn C (2016): Increased regional gray matter atrophy and enhanced functional connectivity in male multiple sclerosis patients. Neurosci Lett 630:154-157. http://dx.doi.org/10.1016/j.neulet.2016.07.028.

Sanislow CA, Ferrante M, Pacheco J, Rudorfer MV, Morris SE (2019): Advancing Translational Research Using NIMH Research Domain Criteria and Computational Methods. Neuron 101:779-782. http://dx.doi.org/10.1016/j.neuron.2019.02.024.

Saveanu R, Etkin A, Duchemin A-M, Goldstein-Piekarski A, Gyurak A, Debattista C, Schatzberg AF, Sood S, Day CVA, Palmer DM, Rekshan WR, Gordon E, Rush AJ, Williams LM (2015): The international Study to Predict Optimized Treatment in Depression (iSPOT-D): outcomes from the acute phase of antidepressant treatment. J Psychiatr Res 61:1-12. http://dx.doi.org/10.1016/j.jpsychires.2014.12.018.

Savica R, Grossardt BR, Bower JH, Boeve BF, Eric Ahlskog J, Rocca WA (2013): Incidence of Dementia With Lewy Bodies and Parkinson Disease Dementia. JAMA Neurol 70:1396-1402. https://jamanetwork.com/journals/jamaneurology/fullarticle/1738443.

Savolainen-Peltonen H, Rahkola-Soisalo P, Hoti F, Vattulainen P, Gissler M, Ylikorkala O, Mikkola TS (2019): Use of postmenopausal hormone therapy and risk of Alzheimer's disease in Finland: nationwide case-control study. BMJ. http://dx.doi.org/10.1136/bmj.1665.

Sawyer KS, Adra N, Salz DM, Kemppainen MI, Ruiz SM, Harris GJ, Oscar-Berman M (2020): Hippocampal subfield volumes in abstinent men and women with a history of alcohol use disorder. PLoS One 15:e0236641. http://dx.doi.org/10.1371/journal.pone.0236641.

Sawyer KS, Maleki N, Papadimitriou G, Makris N, Oscar-Berman M, Harris GJ (2018): Cerebral white matter sex dimorphism in alcoholism: a diffusion tensor imaging study. Neuropsychopharmacology 43:1876-1883. http://dx.doi.org/10.1038/s41386-018-0089-6.

Scanlon C, Mueller SG, Tosun D, Cheong I, Garcia P, Barakos J, Weiner MW, Laxer KD (2011): Impact of methodologic choice for automatic detection of different aspects of brain atrophy by using temporal lobe epilepsy as a model. AJNR Am J Neuroradiol 32:1669-1676. http://dx.doi.org/10.3174/ajnr.A2578.

Schmaal L, Hibar DP, Sämann PG, Hall GB, Baune BT, Jahanshad N, Cheung JW, van Erp TGM, Bos D, Ikram MA, Vernooij MW, Niessen WJ, Tiemeier H, Hofman A, Wittfeld K, Grabe HJ, Janowitz D, Bülow R, Selonke M, Völzke H, Grotegerd D, Dannlowski U, Arolt V, Opel N, Heindel W, Kugel H, Hoehn D, Czisch M, Couvy-Duchesne B, Rentería ME, Strike LT, Wright MJ, Mills NT, de Zubicaray GI, McMahon KL, Medland SE, Martin NG, Gillespie NA, Goya-Maldonado R, Gruber O, Krämer B, Hatton SN, Lagopoulos J, Hickie IB, Frodl T, Carballedo A, Frey EM, van Velzen LS, Penninx BWJH, van Tol M-J, van der Wee NJ, Davey CG, Harrison BJ, Mwangi B, Cao B, Soares JC, Veer IM, Walter H, Schoepf D, Zurowski B, Konrad C, Schramm E, Normann C, Schnell K, Sacchet MD, Gotlib IH, MacQueen GM, Godlewska BR, Nickson T, McIntosh AM, Papmeyer M, Whalley HC, Hall J, Sussmann JE, Li M, Walter M, Aftanas L, Brack I, Bokhan NA, Thompson PM, Veltman DJ (2017): Cortical abnormalities in adults and adolescents with major depression based on brain scans from 20 cohorts worldwide in the ENIGMA Major Depressive Disorder Working Group. Mol Psychiatry 22:900-909. http://dx.doi.org/10.1038/mp.2016.60.

Schmaal L, Veltman DJ, van Erp TGM, Sämann PG, Frodl T, Jahanshad N, Loehrer E, Tiemeier H, Hofman A, Niessen WJ, Vernooij MW, Ikram MA, Wittfeld K, Grabe HJ, Block A, Hegenscheid K, Völzke H, Hoehn D, Czisch M, Lagopoulos J, Hatton SN, Hickie IB, Goya-Maldonado R, Krämer B, Gruber O, Couvy-Duchesne B, Rentería ME, Strike LT, Mills NT, de Zubicaray GI, McMahon KL, 
Medland SE, Martin NG, Gillespie NA, Wright MJ, Hall GB, MacQueen GM, Frey EM, Carballedo A, van Velzen LS, van Tol MJ, van der Wee NJ, Veer IM, Walter H, Schnell K, Schramm E, Normann C, Schoepf D, Konrad C, Zurowski B, Nickson T, McIntosh AM, Papmeyer M, Whalley HC, Sussmann JE, Godlewska BR, Cowen PJ, Fischer FH, Rose M, Penninx BWJH, Thompson PM, Hibar DP (2016): Subcortical brain alterations in major depressive disorder: findings from the ENIGMA Major Depressive Disorder working group. Mol Psychiatry 21:806-812. http://dx.doi.org/10.1038/mp.2015.69.

Schoonheim MM, Popescu V, Rueda Lopes FC, Wiebenga OT, Vrenken H, Douw L, Polman CH, Geurts JJG, Barkhof F (2012): Subcortical atrophy and cognition: sex effects in multiple sclerosis. Neurology 79:1754-1761. http://dx.doi.org/10.1212/WNL.0b013e3182703f46.

Schoonheim MM, Vigeveno RM, Rueda Lopes FC, Pouwels PJW, Polman CH, Barkhof F, Geurts JJG (2014): Sex-specific extent and severity of white matter damage in multiple sclerosis: implications for cognitive decline. Hum Brain Mapp 35:2348-2358. http://dx.doi.org/10.1002/hbm.22332.

Schulz KM, Molenda-Figueira HA, Sisk CL (2009): Back to the future: The organizational-activational hypothesis adapted to puberty and adolescence. Horm Behav 55:597-604. http://dx.doi.org/10.1016/j.yhbeh.2009.03.010.

Schulz KM, Sisk CL (2016): The organizing actions of adolescent gonadal steroid hormones on brain and behavioral development. Neurosci Biobehav Rev 70:148-158. http://dx.doi.org/10.1016/j.neubiorev.2016.07.036.

Schumacher M, Hussain R, Gago N, Oudinet J-P, Mattern C, Ghoumari AM (2012): Progesterone synthesis in the nervous system: implications for myelination and myelin repair. Front Neurosci 6:10. http://dx.doi.org/10.3389/fnins.2012.00010.

Schwarz JM, Bilbo SD (2012): Sex, glia, and development: interactions in health and disease. Horm Behav 62:243-253. http://dx.doi.org/10.1016/j.yhbeh.2012.02.018.

Seedat S, Scott KM, Angermeyer MC, Berglund P, Bromet EJ, Brugha TS, Demyttenaere K, de Girolamo G, Haro JM, Jin R, Karam EG, Kovess-Masfety V, Levinson D, Medina Mora ME, Ono Y, Ormel J, Pennell B-E, Posada-Villa J, Sampson NA, Williams D, Kessler RC (2009): Cross-national associations between gender and mental disorders in the World Health Organization World Mental Health Surveys. Arch Gen Psychiatry 66:785-795. http://dx.doi.org/10.1001/archgenpsychiatry.2009.36.

Seeman MV (2013): Women and schizophrenia: new findings. Neuropsychiatry 3:423. https://pdfs.semanticscholar.org/5f3b/959bb67fe735b656192b3b5763efc4d6ebed.pdf.

Senefeld JW, Lambelet Coleman D, Johnson PW, Carter RE, Clayburn AJ, Joyner MJ (2020): Divergence in Timing and Magnitude of Testosterone Levels Between Male and Female Youths. JAMA 324:99101. http://dx.doi.org/10.1001/jama.2020.5655.

Seo SW, Im K, Lee J-M, Kim ST, Ahn HJ, Go SM, Kim S-H, Na DL (2011): Effects of demographic factors on cortical thickness in Alzheimer's disease. Neurobiol Aging 32:200-209. http://dx.doi.org/10.1016/j.neurobiolaging.2009.02.004.

Serrano-Pozo A, Frosch MP, Masliah E, Hyman BT (2011): Neuropathological alterations in Alzheimer disease. Cold Spring Harb Perspect Med 1:a006189. http://dx.doi.org/10.1101/cshperspect.a006189.

Shao H, Breitner JCS, Whitmer RA, Wang J, Hayden K, Wengreen H, Corcoran C, Tschanz J, Norton M, Munger R, Welsh-Bohmer K, Zandi PP, Cache County Investigators (2012): Hormone therapy and Alzheimer disease dementia: new findings from the Cache County Study. Neurology 79:1846-1852. http://dx.doi.org/10.1212/WNL.0b013e318271f823.

Sheikh JI, Leskin GA, Klein DF (2002): Gender differences in panic disorder: findings from the National Comorbidity Survey. Am J Psychiatry 159:55-58. http://dx.doi.org/10.1176/appi.ajp.159.1.55.

Shirani A, Sun P, Trinkaus K, Perantie DC, George A, Naismith RT, Schmidt RE, Song S-K, Cross AH (2019): Diffusion basis spectrum imaging for identifying pathologies in MS subtypes. Ann Clin Transl Neurol 6:2323-2327. http://dx.doi.org/10.1002/acn3.50903.

Sinforiani E, Citterio A, Zucchella C, Bono G, Corbetta S, Merlo P, Mauri M (2010): Impact of gender differences on the outcome of Alzheimer's disease. Dement Geriatr Cogn Disord 30:147-154. http://dx.doi.org/10.1159/000318842.

Singh-Manoux A, Dugravot A, Fournier A, Abell J, Ebmeier K, Kivimäki M, Sabia S (2017): Trajectories of Depressive Symptoms Before Diagnosis of Dementia: A 28-Year Follow-up Study. JAMA Psychiatry 74:712-718. http://dx.doi.org/10.1001/jamapsychiatry.2017.0660.

Sit D (2004): Women and bipolar disorder across the life span. J Am Med Womens Assoc 59:91-100. 
https://www.ncbi.nlm.nih.gov/pubmed/15134424.

Skup M, Zhu H, Wang Y, Giovanello KS, Lin J-A, Shen D, Shi F, Gao W, Lin W, Fan Y, Zhang H, Alzheimer's Disease Neuroimaging Initiative (2011): Sex differences in grey matter atrophy patterns among $A D$ and aMCI patients: results from ADNI. Neuroimage 56:890-906. http://dx.doi.org/10.1016/j.neuroimage.2011.02.060.

Smith SM, Zhang Y, Jenkinson M, Chen J, Matthews PM, Federico A, De Stefano N (2002): Accurate, robust, and automated longitudinal and cross-sectional brain change analysis. Neuroimage 17:479489. http://dx.doi.org/10.1006/nimg.2002.1040.

Sobanski T, Wagner G (2017): Functional neuroanatomy in panic disorder: Status quo of the research. World J Psychiatry 7:12-33. http://dx.doi.org/10.5498/wjp.v7.i1.12.

Søeborg T, Frederiksen H, Mouritsen A, Johannsen TH, Main KM, Jørgensen N, Petersen JH, Andersson A-M, Juul A (2014): Sex, age, pubertal development and use of oral contraceptives in relation to serum concentrations of DHEA, DHEAS, 17a-hydroxyprogesterone, $\triangle 4$-androstenedione, testosterone and their ratios in children, adolescents and young adults. Clinica Chimica Acta. http://dx.doi.org/10.1016/j.cca.2014.06.018.

Solla P, Cannas A, Ibba FC, Loi F, Corona M, Orofino G, Marrosu MG, Marrosu F (2012): Gender differences in motor and non-motor symptoms among Sardinian patients with Parkinson's disease. J Neurol Sci 323:33-39. http://dx.doi.org/10.1016/j.jns.2012.07.026.

Sporns O (2018): Graph theory methods: applications in brain networks. Dialogues Clin Neurosci 20:111121. https://www.ncbi.nlm.nih.gov/pubmed/30250388.

Substance Abuse and Mental Health Services Administration (2016): TEDS 2004-2014 National Admissions to Substance Abuse Treatment Services. https://wwwdasis.samhsa.gov/dasis2/teds_pubs/2014_teds_rpt_natl.pdf.

Sugimoto CR, Ahn Y-Y, Smith E, Macaluso B, Larivière V (2019): Factors affecting sex-related reporting in medical research: a cross-disciplinary bibliometric analysis. Lancet 393:550-559. http://dx.doi.org/10.1016/S0140-6736(18)32995-7.

Szádóczky E, Papp Z s., Vitrai J, Ríhmer Z, Füredi J (1998): The prevalence of major depressive and bipolar disorders in Hungary. Results from a national epidemiologic survey. J Affect Disord 50:153162. http://dx.doi.org/10.1016/s0165-0327(98)00056-1.

Szucs D, loannidis JPA (2017): Empirical assessment of published effect sizes and power in the recent cognitive neuroscience and psychology literature. PLoS Biol 15:e2000797. http://dx.doi.org/10.1371/journal.pbio.2000797.

Takahashi T, Kawasaki Y, Kurokawa K, Hagino H, Nohara S, Yamashita I, Nakamura K, Murata M, Matsui M, Suzuki M, Seto H, Kurachi M (2002): Lack of normal structural asymmetry of the anterior cingulate gyrus in female patients with schizophrenia: a volumetric magnetic resonance imaging study. Schizophr Res 55:69-81. http://dx.doi.org/10.1016/s0920-9964(01)00200-6.

Takayanagi Y, Takahashi T, Orikabe L, Mozue Y, Kawasaki Y, Nakamura K, Sato Y, Itokawa M, Yamasue H, Kasai K, Others (2011): Classification of first-episode schizophrenia patients and healthy subjects by automated MRI measures of regional brain volume and cortical thickness. PLoS One 6. https://www.ncbi.nlm.nih.gov/pmc/articles/pmc3119676/.

Tan A, Ma W, Vira A, Marwha D, Eliot L (2016): The human hippocampus is not sexually-dimorphic: Meta-analysis of structural MRI volumes. Neuroimage 124:350-366. http://dx.doi.org/10.1016/j.neuroimage.2015.08.050.

Taylor KSM, Cook JA, Counsell CE (2007): Heterogeneity in male to female risk for Parkinson's disease. Journal of neurology, neurosurgery, and psychiatry. http://dx.doi.org/10.1136/jnnp.2006.104695.

Terlizzi EP, Villarroel MA (2020): Symptoms of Generalized Anxiety Disorder Among Adults: United States, 2019. NCHS Data Brief:1-8. https://www.ncbi.nlm.nih.gov/pubmed/33054928.

The National Institute of Mental Health (2020): Bipolar Disorder. Bipolar Disorder. https://www.nimh.nih.gov/health/topics/bipolar-disorder/index.shtml.

Thompson PM, Giedd JN, Woods RP, MacDonald D, Evans AC, Toga AW (2000): Growth patterns in the developing brain detected by using continuum mechanical tensor maps. Nature 404:190-193. http://dx.doi.org/10.1038/35004593.

Thompson PM, Jahanshad N, Ching CRK, Salminen LE, Thomopoulos SI, Bright JK, Baune BT, Bertolín S, Bralten J, Bruin WB, Bülow R, Chen J, Chye Y, Dannlowski U, de Kovel CGF, Donohoe G, Eyler LT, Faraone SV, Favre P, Filippi CA, Frodl T, Garijo D, Gil Y, Grabe HJ, Grasby KL, Hajek T, Han LKM, Hatton SN, Hilbert K, Ho TC, Holleran L, Homuth G, J HN, Ivanov I, Jia T, Kelly S, Klein M, 
Kwon JS, Laansma MA, Leerssen J, Lueken U, Nunes A, O'Neill J, Opel N, Piras F PF, Postema MC, Pozzi E, Shatokhina N, Soriano-Mas C, Spalletta G, Sun D, Teumer A, Tilot AK, Tozzi L, van der Merwe C, Van Someren EJW, van Wingen GA, Völzke H, Walton E, Wang L, Winkler AM, Wittfeld K, Wright MJ, J-Y Y, Zhang G, Zhang-James Y, Adhikari BM, Agartz I, Aghajani M, Aleman A, Althoff RR, Altmann A, Andreassen OA, Baron DA, Bartnik-Olson BL, Baskin-Sommers AR, Bearden CE, Berner LA, Boedhoe PSW, Brouwer RM, Buitelaar JK, Caeyenberghs K, Cecil CAM, Cohen RA, Cole JH, Conrod PJ, De Brito SA, de Zwarte SMC, Dennis EL, Desrivieres S, Dima D, Ehrlich S, Esopenko C, Fairchild G, Fisher SE, J-P F, Francks C, Frangou S, Franke B, Garavan HP, Glahn DC, Groenewold NA, Gurholt TP, Gutman BA, Hahn T, Harding IH, Hernaus D, Hibar DP, Hillary FG, Hoogman M, He HP, Jalbrzikowski M, Karkashadze G, Klapwijk ET, Knickmeyer RC, Kochunov P, Koerte IK, X-K K, S-L L, Lin AP, Logue MW, Lueders E, Macciardi F, Mackey S, Mayer AR, McDonald CR, McMahon AB, Medland SE, Modinos G, Morey RA, Mueller SC, Mukherjee P, Namazova-Baranova L, Nir TM, Olsen A, Paschou P, Pine DS, Pizzagalli F, Rentería ME, Rohrer JD, Sämann PG, Schmaal L, Schumann G, Shiroishi MS, Sisodiya SM, Smit DJA, Sønderby IE, Stein DJ, Stein JL, Mahmasian MT, Tate DF, Turner JA, van den Heuvel OA, van der Wee NJA, van der Werf YD, van Erp TGM, van Haren NEM, van Rooij D, van Velzen LS, Veer IM, Veltman DJ, VillalonReina JE, Walter H, Whelan CD, Wilde EA, Zarei M, Zelman V (2020): ENIGMA and Global Neuroscience: A Decade of Large-Scale Studies of the Brain in Health and Disease across 43 Countries. Transl Psychiatry.

Tolaymat B, Zheng W, Chen H, Choi S, Li X, Harrison DM (2020): Sex-specific differences in rim appearance of multiple sclerosis lesions on quantitative susceptibility mapping. Mult Scler Relat Disord 45:102317. http://dx.doi.org/10.1016/j.msard.2020.102317.

Tomlinson C, Macintyre H, Dorrian CA, Ahmed SF, Wallace AM (2004): Testosterone measurements in early infancy. Arch Dis Child Fetal Neonatal Ed 89:F558-9. http://dx.doi.org/10.1136/adc.2003.034017.

Torresan RC, Ramos-Cerqueira ATA, Shavitt RG, do Rosário MC, de Mathis MA, Miguel EC, Torres AR (2013): Symptom dimensions, clinical course and comorbidity in men and women with obsessivecompulsive disorder. Psychiatry Res 209:186-195. http://dx.doi.org/10.1016/j.psychres.2012.12.006.

Tozzi L, Garczarek L, Janowitz D, Stein DJ, Wittfeld K, Dobrowolny H, Lagopoulos J, Hatton SN, Hickie IB, Carballedo A, Brooks SJ, Vuletic D, Uhlmann A, Veer IM, Walter H, Bülow R, Völzke H, KlingerKönig J, Schnell K, Schoepf D, Grotegerd D, Opel N, Dannlowski U, Kugel H, Schramm E, Konrad C, Kircher T, Jüksel D, Nenadić I, Krug A, Hahn T, Steinsträter O, Redlich R, Zaremba D, Zurowski B, Fu CHY, Dima D, Cole J, Grabe HJ, Connolly CG, Yang TT, Ho TC, LeWinn KZ, Li M, Groenewold NA, Salminen LE, Walter M, Simmons AN, van Erp TGM, Jahanshad N, Baune BT, van der Wee NJA, van Tol M-J, Penninx BWJH, Hibar DP, Thompson PM, Veltman DJ, Schmaal L, Frodl T, "for the ENIGMA-MDD Consortium" (2019): Interactive impact of childhood maltreatment, depression, and age on cortical brain structure: mega-analytic findings from a large multi-site cohort. Psychol Med:1-12. http://dx.doi.org/10.1017/S003329171900093X.

Tremblay C, Abbasi N, Zeighami Y, Yau Y, Dadar M, Rahayel S, Dagher A (2020): Sex effects on brain structure in de novo Parkinson's disease: a multimodal neuroimaging study. Brain 143:3052-3066. http://dx.doi.org/10.1093/brain/awaa234.

Truelsen T, Thudium D, Grønbaek M, Copenhagen City Heart Study (2002): Amount and type of alcohol and risk of dementia: the Copenhagen City Heart Study. Neurology 59:1313-1319. http://dx.doi.org/10.1212/01.wnl.0000031421.50369.e7.

Tutuncu M, Tang J, Zeid NA, Kale N, Crusan DJ, Atkinson EJ, Siva A, Pittock SJ, Pirko I, Keegan BM, Lucchinetti CF, Noseworthy JH, Rodriguez M, Weinshenker BG, Kantarci OH (2013): Onset of progressive phase is an age-dependent clinical milestone in multiple sclerosis. Mult Scler 19:188198. http://dx.doi.org/10.1177/1352458512451510.

Tyagi V, Scordo M, Yoon RS, Liporace FA, Greene LW (2017): Revisiting the role of testosterone: Are we missing something? Rev Urol 19:16-24. http://dx.doi.org/10.3909/riu0716.

Uchida RR, Del-Ben CM, Busatto GF, Duran FLS, Guimarães FS, Crippa JAS, Araújo D, Santos AC, Graeff FG (2008): Regional gray matter abnormalities in panic disorder: a voxel-based morphometry study. Psychiatry Res 163:21-29. http://dx.doi.org/10.1016/j.pscychresns.2007.04.015.

van Dijk Ewoud, Prins Niels D., Vrooman Henri A., Hofman Albert, Koudstaal Peter J., Breteler Monique M.B. (2008): Progression of Cerebral Small Vessel Disease in Relation to Risk Factors and Cognitive Consequences. Stroke 39:2712-2719. https://doi.org/10.1161/STROKEAHA.107.513176. 
Van Gestel H, Franke K, Petite J, Slaney C, Garnham J, Helmick C, Johnson K, Uher R, Alda M, Hajek T (2019): Brain age in bipolar disorders: Effects of lithium treatment. Aust N Z J Psychiatry 53:1179_ 1188. http://dx.doi.org/10.1177/0004867419857814.

Varma AR, Snowden JS, Lloyd JJ, Talbot PR, Mann DM, Neary D (1999): Evaluation of the NINCDSADRDA criteria in the differentiation of Alzheimer's disease and frontotemporal dementia. J Neurol Neurosurg Psychiatry 66:184-188. http://dx.doi.org/10.1136/jnnp.66.2.184.

van Velzen LS, Kelly S, Isaev D, Aleman A, Aftanas LI, Bauer J, Baune BT, Brak IV, Carballedo A, Connolly CG, Couvy-Duchesne B, Cullen KR, Danilenko KV, Dannlowski U, Enneking V, Filimonova E, Förster K, Frodl T, Gotlib IH, Groenewold NA, Grotegerd D, Harris MA, Hatton SN, Hawkins EL, Hickie IB, Ho TC, Jansen A, Kircher T, Klimes-Dougan B, Kochunov P, Krug A, Lagopoulos J, Lee R, Lett TA, Li M, MacMaster FP, Martin NG, McIntosh AM, McLellan Q, Meinert S, Nenadic I, Osipov E, Brenda W J, Portella MJ, Repple J, Roos A, Sacchet MD, Sämann PG, Schnell K, Shen X, Sim K, Stein DJ, van Tol M-J, Tomyshev AS, Tozzi L, Veer IM, Vermeiren R, Vives-Gilabert Y, Walter H, Walter M, van der Wee NJA, van der Werff SJA, Schreiner MW, Whalley HC, Wright MJ, Yang TT, Zhu A, Veltman DJ, Thompson PM, Jahanshad\# N, Schmaal L (2019): White matter disturbances in major depressive disorder: a coordinated analysis across 20 international cohorts in the ENIGMA MDD working group. Mol Psychiatry.

Ventura-Clapier R, Piquereau J, Veksler V, Garnier A (2019): Estrogens, Estrogen Receptors Effects on Cardiac and Skeletal Muscle Mitochondria. Front Endocrinol 10:557. http://dx.doi.org/10.3389/fendo.2019.00557.

Virani SS, Alonso A, Benjamin EJ, Bittencourt MS, Callaway CW, Carson AP, Chamberlain AM, Chang AR, Cheng S, Delling FN, Djousse L, Elkind MSV, Ferguson JF, Fornage M, Khan SS, Kissela BM, Knutson KL, Kwan TW, Lackland DT, Lewis TT, Lichtman JH, Longenecker CT, Loop MS, Lutsey PL, Martin SS, Matsushita K, Moran AE, Mussolino ME, Perak AM, Rosamond WD, Roth GA, Sampson UKA, Satou GM, Schroeder EB, Shah SH, Shay CM, Spartano NL, Stokes A, Tirschwell DL, VanWagner LB, Tsao CW, On behalf of the American Heart Association Council on Epidemiology and Prevention Statistics Committee and Stroke Statistics Subcommittee (2020): Heart Disease and Stroke Statistics-2020 Update. Circulation. http://dx.doi.org/10.1161/cir.0000000000000757.

Voigt J, Pakkenberg H (1983): Brain Weight of Danish Children. Cells Tissues Organs 116:290-301. https://www.karger.com/DOI/10.1159/000145754.

Voskuhl RR, Patel K, Paul F, Gold SM, Scheel M, Kuchling J, Cooper G, Asseyer S, Chien C, Brandt AU, Meyer CE, MacKenzie-Graham A (2020): Sex differences in brain atrophy in multiple sclerosis. Biol Sex Differ 11:49. http://dx.doi.org/10.1186/s13293-020-00326-3.

Vyas DA, Eisenstein LG, Jones DS (2020): Hidden in Plain Sight - Reconsidering the Use of Race Correction in Clinical Algorithms. N Engl J Med 383:874-882. https://doi.org/10.1056/NEJMms2004740.

Waller M, Mishra GD, Dobson AJ (2017): Estimating the prevalence of dementia using multiple linked administrative health records and capture-recapture methodology. Emerg Themes Epidemiol 14:3. http://dx.doi.org/10.1186/s12982-017-0057-3.

Wallin MT, Culpepper WJ, Nichols E, Bhutta ZA, Gebrehiwot TT, Hay SI, Khalil IA, Krohn KJ, Liang X, Naghavi M, Mokdad AH, Nixon MR, Reiner RC, Sartorius B, Smith M, Topor-Madry R, Werdecker A, Vos T, Feigin VL, Murray CJL (2019): Global, regional, and national burden of multiple sclerosis 1990-2016: a systematic analysis for the Global Burden of Disease Study 2016. Lancet Neurol 18:269-285. http://www.sciencedirect.com/science/article/pii/S1474442218304435.

Walther A, Wasielewska JM, Leiter O (2019): The antidepressant effect of testosterone: An effect of neuroplasticity? Neurology, Psychiatry and Brain Research 32:104-110. http://www.sciencedirect.com/science/article/pii/S0941950019300119.

Walton C, King R, Rechtman L, Kaye W, Leray E, Marrie RA, Robertson N, La Rocca N, Uitdehaag B, van der Mei I, Wallin M, Helme A, Angood Napier C, Rijke N, Baneke P (2020): Rising prevalence of multiple sclerosis worldwide: Insights from the Atlas of MS, third edition. Mult Scler 26:1816-1821. http://dx.doi.org/10.1177/1352458520970841.

Wang GH (1923): The Relation Between" Spontaneous" Activity and Oestrous Cycle in the White Rat. Comparative Psychology Monographs. https://psycnet.apa.org/record/1926-07707-001.

Wang X, Xie H, Chen T, Cotton AS, Salminen LE, Logue MW, Clarke-Rubright EK, Wall J, Dennis EL, O'Leary BM, Abdallah CG, Andrew E, Baugh LA, Bomyea J, Bruce SE, Bryant R, Choi K, Daniels JK, Davenport ND, Davidson RJ, DeBellis M, deRoon-Cassini T, Disner SG, Fani N, Fercho KA, 
Fitzgerald J, Forster GL, Frijling JL, Geuze E, Gomaa H, Gordon EM, Grupe D, Harpaz-Rotem I, Haswell CC, Herzog JI, Hofmann D, Hollifield M, Hosseini B, Hudson AR, Ipser J, Jahanshad N, Jovanovic T, Kaufman ML, King AP, Koch SBJ, Koerte IK, Korgaonkar MS, Krystal JH, Larson C, Lebois LAM, Levy I, Li G, Magnotta VA, Manthey A, May G, McLaughlin KA, Mueller SC, Nawijn L, Nelson SM, Neria Y, Nitschke JB, Olff M, Olson EA, Peverill M, Luan Phan K, Rashid FM, Ressler K, Rosso IM, Sambrook K, Schmahl C, Shenton ME, Sierk A, Simons JS, Simons RM, Sponheim SR, Stein MB, Stein DJ, Stevens JS, Straube T, Suarez-Jimenez B, Tamburrino M, Thomopoulos SI, van der Wee NJA, van der Werff SJA, van Erp TGM, van Rooij SJH, van Zuiden M, Varkevisser T, Veltman DJ, Vermeiren RRJM, Walter H, Wang L, Zhu Y, Zhu X, Thompson PM, Morey RA, Liberzon I (2020a): Cortical volume abnormalities in posttraumatic stress disorder: an ENIGMA-psychiatric genomics consortium PTSD workgroup mega-analysis. Mol Psychiatry. http://dx.doi.org/10.1038/s41380-020-00967-1.

Wang X, Cheng B, Wang S, Lu F, Luo Y, Long X, Kong D (2020b): Distinct grey matter volume alterations in adult patients with panic disorder and social anxiety disorder: A systematic review and voxel-based morphometry meta-analysis. J Affect Disord. http://www.sciencedirect.com/science/article/pii/S0165032720329876.

Wang X, Luo Q, Tian F, Cheng B, Qiu L, Wang S, He M, Wang H, Duan M, Jia Z (2019): Brain greymatter volume alteration in adult patients with bipolar disorder under different conditions: a voxelbased meta-analysis. J Psychiatry Neurosci 44:89-101. http://dx.doi.org/10.1503/jpn.180002.

Ward JB, Gartner DR, Keyes KM, Fliss MD, McClure ES, Robinson WR (2019): How do we assess a racial disparity in health? Distribution, interaction, and interpretation in epidemiological studies. Ann Epidemiol 29:1-7. http://dx.doi.org/10.1016/j.annepidem.2018.09.007.

Wardlaw JM, Valdés Hernández MC, Muñoz-Maniega S (2015): What are white matter hyperintensities made of? Relevance to vascular cognitive impairment. J Am Heart Assoc 4:001140. http://dx.doi.org/10.1161/JAHA.114.001140.

Weiser MJ, Foradori CD, Handa RJ (2008): Estrogen receptor beta in the brain: from form to function. Brain Res Rev 57:309-320. http://dx.doi.org/10.1016/j.brainresrev.2007.05.013.

Westbury C, Bub D (1997): Primary progressive aphasia: a review of 112 cases. Brain Lang 60:381-406. http://dx.doi.org/10.1006/brln.1997.1840.

White H, Boden-Albala B, Wang C, Elkind MSV, Rundek T, Wright CB, Sacco RL (2005): Ischemic stroke subtype incidence among whites, blacks, and Hispanics: the Northern Manhattan Study. Circulation 111:1327-1331. http://dx.doi.org/10.1161/01.CIR.0000157736.19739.D0.

Wierenga LM, Doucet GE, Dima D, Agartz I, Aghajani M, Akudjedu TN, Albajes-Eizagirre A, Alnaes D, Alpert KI, Andreassen OA, Anticevic A, Asherson P, Banaschewski T, Bargallo N, Baumeister S, Baur-Streubel R, Bertolino A, Bonvino A, Boomsma DI, Borgwardt S, Bourque J, den Braber A, Brandeis D, Breier A, Brodaty H, Brouwer RM, Buitelaar JK, Busatto GF, Calhoun VD, CanalesRodríguez EJ, Cannon DM, Caseras X, Castellanos FX, Chaim-Avancini TM, Ching CR, Clark VP, Conrod PJ, Conzelmann A, Crivello F, Davey CG, Dickie EW, Ehrlich S, Van't Ent D, Fisher SE, Fouche J-P, Franke B, Fuentes-Claramonte P, de Geus EJ, Di Giorgio A, Glahn DC, Gotlib IH, Grabe HJ, Gruber O, Gruner P, Gur RE, Gur RC, Gurholt TP, de Haan L, Haatveit B, Harrison BJ, Hartman CA, Hatton SN, Heslenfeld DJ, van den Heuvel OA, Hickie IB, Hoekstra PJ, Hohmann S, Holmes AJ, Hoogman M, Hosten N, Howells FM, Hulshoff Pol HE, Huyser C, Jahanshad N, James AC, Jiang J, Jönsson EG, Joska JA, Kalnin AJ, Karolinska Schizophrenia Project (KaSP) Consortium, Klein M, Koenders L, Kolskår KK, Krämer B, Kuntsi J, Lagopoulos J, Lazaro L, Lebedeva IS, Lee PH, Lochner C, Machielsen MW, Maingault S, Martin NG, Martínez-Zalacaín I, Mataix-Cols D, Mazoyer B, McDonald BC, McDonald C, McIntosh AM, McMahon KL, McPhilemy G, van der Meer D, Menchón JM, Naaijen J, Nyberg L, Oosterlaan J, Paloyelis Y, Pauli P, Pergola G, Pomarol-Clotet E, Portella MJ, Radua J, Reif A, Richard G, Roffman JL, Rosa PG, Sacchet MD, Sachdev PS, Salvador R, Sarró S, Satterthwaite TD, Saykin AJ, Serpa MH, Sim K, Simmons A, Smoller JW, Sommer IE, SorianoMas C, Stein DJ, Strike LT, Szeszko PR, Temmingh HS, Thomopoulos SI, Tomyshev AS, Trollor JN, Uhlmann A, Veer IM, Veltman DJ, Voineskos A, Völzke H, Walter H, Wang L, Wang Y, Weber B, Wen W, West JD, Westlye LT, Whalley HC, Williams SC, Wittfeld K, Wolf DH, Wright MJ, Yoncheva YN, Zanetti MV, Ziegler GC, de Zubicaray GI, Thompson PM, Crone EA, Frangou S, Tamnes CK (2020a): Greater male than female variability in regional brain structure across the lifespan. Hum Brain Mapp. http://dx.doi.org/10.1002/hbm.25204.

Wierenga LM, Doucet GE, Dima D, Agartz I, Aghajani M, Akudjedu TN, Albajes-Eizagirre A, Alnæs D, 
Alpert KI, Andreassen OA, Anticevic A, Asherson P, Banaschewski T, Bargallo N, Baumeister S, Baur-Streubel R, Bertolino A, Bonvino A, Boomsma DI, Borgwardt S, Bourque J, den Braber A, Brandeis D, Breier A, Brodaty H, Brouwer RM, Busatto GF, Calhoun VD, Canales-Rodríguez EJ, Cannon DM, Caseras X, Chaim-Avancini TM, Ching CRK, Conrod PJ, Conzelmann A, Crivello F, Davey CG, Dickie EW, Ehrlich S, Ent DV, Fouche J-P, Fuentes-Claramonte P, de Geus EJC, Di Giorgio A, Glahn DC, Gotlib IH, Grabe HJ, Gruber O, Gruner P, Gur RE, Gur RC, Gurholt TP, de Haan L, Haatveit B, Harrison BJ, Hatton SN, van den Heuvel OA, Hickie IB, Hohmann S, Holmes AJ, Hoogman M, Hosten N, Howells FM, Hulshoff Pol HE, Huyser C, Jahanshad N, James AC, Jönsson EG, Joska JA, Karolinska Schizophrenia Project (KaSP) Consortium, Klein M, Koenders L, Kolskår KK, Krämer B, Kuntsi J, Lagopoulos J, Lazaro L, Lebedeva IS, Lee PH, Lochner C, Machielsen MWJ, Maingault S, Martin NG, Martínez-Zalacaín I, Mataix-Cols D, Mazoyer B, McDonald BC, McDonald C, McIntosh AM, McMahon KL, McPhilemy G, van der Meer D, Menchón JM, Naaijen J, Nyberg L, Paloyelis Y, Pauli P, Pergola G, Pomarol-Clotet E, Portella MJ, Radua J, Reif A, Richard G, Roffman JL, Rosa PGP, Sacchet MD, Sachdev PS, Salvador R, Sarró S, Satterthwaite TD, Saykin AJ, Serpa MH, Sim K, Simmons A, Smoller JW, Sommer IE, Soriano-Mas C, Stein DJ, Strike LT, Szeszko PR, Temmingh HS, Thomopoulos SI, Tomyshev AS, Trollor JN, Uhlmann A, Veer IM, Veltman DJ, Voineskos A, Völzke H, Walter H, Wang L, Wang Y, Weber B, Wen W, West JD, Westlye LT, Whalley HC, Williams SCR, Wittfeld K, Wolf DH, Wright MJ, Yoncheva YN, Zanetti MV, Ziegler GC, de Zubicaray GI, Thompson PM, Crone EA, Frangou S, Tamnes CK (2020b): Greater male than female variability in regional brain structure across the lifespan. biorxiv. https://www.biorxiv.org/content/10.1101/2020.02.17.952010v1.full.

Wilkins CH, Schindler SE, Morris JC (2020): Addressing Health Disparities Among Minority Populations: Why Clinical Trial Recruitment Is Not Enough. JAMA Neurol 77:1063-1064. http://dx.doi.org/10.1001/jamaneurol.2020.1614.

Witte AV, Savli M, Holik A, Kasper S, Lanzenberger R (2010): Regional sex differences in grey matter volume are associated with sex hormones in the young adult human brain. Neuroimage 49:12051212. http://dx.doi.org/10.1016/j.neuroimage.2009.09.046.

Wolf EJ, Logue MW, Stoop TB, Schichman SA, Stone A, Sadeh N, Hayes JP, Miller MW (2017): Accelerated DNA Methylation Age: Associations with PTSD and Mortality. Psychosom Med. http://dx.doi.org/10.1097/PSY.0000000000000506.

Womer FY, Tang Y, Harms MP, Bai C, Chang M, Jiang X, Wei S, Wang F, Barch DM (2016): Sexual dimorphism of the cerebellar vermis in schizophrenia. Schizophr Res 176:164-170. http://dx.doi.org/10.1016/j.schres.2016.06.028.

World Health Organization. (2017): Depression and other common mental disorders: global health estimates. https://apps.who.int/iris/bitstream/handle/10665/254610/WHO-MSD-MER-2017.2eng.pdf?sequence=1\&isAllowed=y.

Wu M, Li M, Yuan J, Liang S, Chen Z, Ye M, Ryan PM, Clark C, Tan SC, Rahmani J, Varkaneh HK, Bhagavathula AS (2020): Postmenopausal hormone therapy and Alzheimer's disease, dementia, and Parkinson's disease: A systematic review and time-response meta-analysis. Pharmacol Res 155:104693. http://dx.doi.org/10.1016/j.phrs.2020.104693.

Yaffe K, Vittinghoff E, Lindquist K, Barnes D, Covinsky KE, Neylan T, Kluse M, Marmar C (2010): Posttraumatic stress disorder and risk of dementia among US veterans. Arch Gen Psychiatry 67:608-613. http://dx.doi.org/10.1001/archgenpsychiatry.2010.61.

Yagi S, Galea LAM (2019): Sex differences in hippocampal cognition and neurogenesis. Neuropsychopharmacology 44:200-213. http://dx.doi.org/10.1038/s41386-018-0208-4.

Yang G, Bozek J, Han M, Gao J-H (2020): Constructing and evaluating a cortical surface atlas and analyzing cortical sex differences in young Chinese adults. Hum Brain Mapp. http://dx.doi.org/10.1002/hbm.24960.

Yare K, Woodward M (2020): Hormone Therapy and Effects on Sporadic Alzheimer's Disease in Postmenopausal Women: Importance of Nomenclature. Journal of Alzheimer's Disease. http://dx.doi.org/10.3233/jad-190896.

Yonkers KA, Bruce SE, Dyck IR, Keller MB (2003): Chronicity, relapse, and illness--course of panic disorder, social phobia, and generalized anxiety disorder: findings in men and women from 8 years of follow-up. Depress Anxiety 17:173-179. http://dx.doi.org/10.1002/da.10106.

Young LJ, Pfaff DW (2014): Sex differences in neurological and psychiatric disorders. Front Neuroendocrinol 35:253-254. http://dx.doi.org/10.1016/j.yfrne.2014.05.005. 
Zarow C, Lyness SA, Mortimer JA, Chui HC (2003): Neuronal loss is greater in the locus coeruleus than nucleus basalis and substantia nigra in Alzheimer and Parkinson diseases. Arch Neurol 60:337-341. http://dx.doi.org/10.1001/archneur.60.3.337.

Zeydan B, Kantarci OH (2020): Impact of Age on Multiple Sclerosis Disease Activity and Progression. Curr Neurol Neurosci Rep 20:24. http://dx.doi.org/10.1007/s11910-020-01046-2.

Zhang J, Zhang Y-T, Hu W-D, Li L, Liu G-Y, Bai Y-P (2015): Gray matter atrophy in patients with Parkinson's disease and those with mild cognitive impairment: a voxel-based morphometry study. Int J Clin Exp Med 8:15383-15392. https://www.ncbi.nlm.nih.gov/pubmed/26629027.

Zilkens RR, Bruce DG, Duke J, Spilsbury K, Semmens JB (2014): Severe psychiatric disorders in mid-life and risk of dementia in late- life (age 65-84 years): a population based case-control study. Curr Alzheimer Res 11:681-693. http://dx.doi.org/10.2174/1567205011666140812115004.

Zucker I, Beery AK (2010): Males still dominate animal studies. Nature 465:690. http://dx.doi.org/10.1038/465690a.

Zucker I, Beery AK (2019): Studying Sex as a Biological Variable: Is a New Day Dawning? Journal of women's health . http://dx.doi.org/10.1089/jwh.2018.7598.

Boyle CP, Raji CA, Erickson KI, Lopez OL, Becker JT, Gach HM, Longstreth WT, Popov M, Carmichael OT, Riedel BC, Thompson PM (In Press). Estrogen, Brain Structure, and Cognition in PostMenopausal Women, Human Brain Mapping.

Grace S, Rossetti MG, Allen N, Batalla A, Bellani M, Brambilla P, Chye Y, Conrod P, Cousijn J, Garavan H, Goudriaan AE, Hester R, Hutchison K, Labuschagne I, Momenan R, Mackey S, Martin-Santos R, Patalay P, Rendell P, Solowij N, Sinha R, Schmaal L, Sjoerds Z, Suo C, Terrett G, Thompson PM, van Holst R, Veltman D, Yücel M, the ENIGMA Addiction Working Group, Lorenzetti, V (In Press). Sex differences in the neuroanatomy of alcohol dependence: hippocampus and amygdala subregions in a sample of 966 people from the ENIGMA Addiction Working Group, Biological Psychiatry. 


\begin{tabular}{|c|c|c|c|c|c|c|c|c|}
\hline \multicolumn{3}{|c|}{ Psychiatric Diseases } & & & \multicolumn{4}{|c|}{ Subtypes } \\
\hline Disease & \multicolumn{2}{|c|}{ Male/Female Proportion of Disease } & $\begin{array}{l}\text { Sex Ratio } \\
\text { (F:M) }\end{array}$ & $\begin{array}{c}\text { Weighted } \\
\text { Sex Ratio } \\
\text { (F:M) }\end{array}$ & Subtype & \multicolumn{2}{|c|}{$\begin{array}{l}\text { Male/Female } \\
\text { Proportion of } \\
\text { Disease }\end{array}$} & $\begin{array}{l}\text { Sex Ratio } \\
\text { (F:M) }\end{array}$ \\
\hline PTSD1 & \multirow{2}{*}{$71 \%$} & \multirow{2}{*}{$29 \%$} & \multirow{2}{*}{$2.5: 1$} & \multirow{2}{*}{$2.3: 1$} & \multirow{2}{*}{$\begin{array}{c}\text { Melancholic }^{5} \\
\text { Anxious }^{5} \\
\end{array}$} & \multirow{2}{*}{$61 \%$} & \multirow{2}{*}{\begin{tabular}{|c|}
$39 \%$ \\
$34 \%$ \\
\end{tabular}} & $1.6: 1$ \\
\hline & & & & & & & & $2.0: 1$ \\
\hline $\mathrm{OCD}^{2}$ & $73 \%$ & $27 \%$ & $2.7: 1$ & $2.0: 1$ & Atypical $^{5}$ & $70 \%$ & $30 \%$ & $2.3: 1$ \\
\hline $\mathrm{GAD}^{2}$ & $69 \%$ & $31 \%$ & $2.2: 1$ & $1.7: 1$ & $\mathrm{BD} \mathrm{I}^{2}$ & $66 \%$ & $34 \%$ & $1.9: 1$ \\
\hline GAD & 00 & 8110 & 2.2 .1 & 1.1 .1 & $\mathrm{BD} \|^{2}$ & $64 \%$ & $36 \%$ & $1.8: 1$ \\
\hline PANIC $^{2}$ & $74 \%$ & $26 \%$ & $2.8: 1$ & $2.1: 1$ & Cocaine $^{3}$ & $36 \%$ & $64 \%$ & $0.6: 1$ \\
\hline MDD $^{3}$ & $65 \%$ & $35 \%$ & $1.8: 1$ & $1.8: 1$ & Alcohol $^{3}$ & $36 \%$ & $64 \%$ & $0.6: 1$ \\
\hline & & & & & Cannabis $^{3}$ & $37 \%$ & $63 \%$ & $0.6: 1$ \\
\hline $\mathrm{BD}^{3}$ & $52 \%$ & $48 \%$ & $1.1: 1$ & $1.0: 1$ & Opiates $^{3}$ & $49 \%$ & $51 \%$ & $0.9: 1$ \\
\hline $\operatorname{sCZ}^{3}$ & $51 \%$ & $9 \%$ & $1.0: 1$ & $1.0: 1$ & Amphetamine $^{3}$ & $64 \%$ & $36 \%$ & $0.8: 1$ \\
\hline suD 3 & & & & & RRMS $^{6}$ & $73 \%$ & $27 \%$ & $2.7: 1$ \\
\hline SUD' & $41 \%$ & & $0.7: 1$ & $0.7: 1$ & SPMS $^{6}$ & $69 \%$ & $31 \%$ & $2.3: 1$ \\
\hline & & & & & RPMS $^{6}$ & $56 \%$ & $44 \%$ & $1.3: 1$ \\
\hline $\mathbf{P D}^{3}$ & $42 \%$ & & $0.7: 1$ & $0.7: 1$ & $\mathrm{PPMS}^{6}$ & $55 \%$ & $45 \%$ & $1.2: 1$ \\
\hline MS $^{3}$ & $72 \%$ & $28 \%$ & $2.6: 1$ & $2.3: 1$ & $\begin{array}{l}\text { 1. National } \\
2010 ; 12\end{array}$ & $\begin{array}{l}\text { tressful Ev } \\
\text { month prev }\end{array}$ & $\begin{array}{l}\text { nts Surve } \\
\text { lence }\end{array}$ & \\
\hline $\mathbf{A D}^{4}$ & $68 \%$ & $32 \%$ & $2.1: 1$ & $1.6: 1$ & & $\begin{array}{l}\text { al Comorbi } \\
\text { month preve }\end{array}$ & & Replication \\
\hline VAD $^{4}$ & $64 \%$ & $36 \%$ & $1.8: 1$ & $1.3: 1$ & 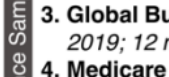 & $\begin{array}{l}\text { den of Dis } \\
\text { onth preval }\end{array}$ & $\begin{array}{l}\text { ase } \\
\text { nce }\end{array}$ & iaries \\
\hline FTD $^{4}$ & $56 \%$ & $44 \%$ & 1.3: 1 & $0.9: 1$ & $\begin{array}{l}\text { 4. Mealcare } \\
2011-201 \\
\text { 5. Enrolled } \mathrm{i}\end{array}$ & $\begin{array}{l}3 \text { year pre } \\
\text { STAR }^{\star} D \text { N }\end{array}$ & $\begin{array}{l}\text { alence } \\
\text { DD trial }\end{array}$ & \\
\hline DLB $^{4}$ & $50 \%$ & $5 \%$ & $1.0: 1$ & $0.7: 1$ & $\begin{array}{r}2001-20 \\
\text { 6. Enrolled i }\end{array}$ & MSBase $\mathrm{F}$ & egistry & \\
\hline Neurod & jenerative & & & & $1951-201$ & Male & & \\
\hline
\end{tabular}

Figure 1. Sex composition of common neurological and psychiatric disorders in males and females in the United States. Male/female proportion of disease, where percentages reflect the male (dark blue) and female (green) proportion of the reference sample with each disease. The dashed line indicates equal proportion of males and females with disease (i.e., 50/50). Sex ratio reflects the absolute number of females with disease for every one male with disease within each sample, which was calculated by dividing the number of females with each disease by the number of males with each disease for each reference sample. The weighted sex ratio reflects the adjusted sex ratio after accounting for male and female differences in sample size. It was calculated by dividing the female disease prevalence (i.e., $\mathrm{N}$ females with disease/ $\mathrm{N}$ total females) by the male disease prevalence (i.e., $\mathrm{N}$ males with disease/ $\mathrm{N}$ total males) from each reference sample. Proportions and ratios do not reflect base rates of disease. Reference samples: Post-traumatic stress disorder (PTSD) calculations are based on 12-month estimates from the 2010 National Stressful Events Survey using DSM-V criteria ( $\mathrm{N}=2953$, ages > 18 years)( $\mathrm{N}=36,309$ adults) [Kilpatrick et al., 2013]. Obsessive compulsive disorder (OCD), generalized anxiety disorder (GAD) and panic disorder (PANIC) calculations were obtained from the National Comorbidity Survey Replication (NCS-R) $(N=9,282$, ages $>13$ years) [Kessler et al., 2012] Major depressive disorder (MDD), bipolar disorder (BD), schizophrenia (SCZ), substance use disorders (SUDs), Parkinson's disease (PD), and multiple sclerosis (MS) calculations were taken from the 2019 Global Burden of Diseases (GBD) study based on 12-month point estimates for the United States. Dementia subtype (Alzheimer's disease (AD), vascular dementia (VAD), frontotemporal lobe dementia (FTD), and dementia with lewy bodies (DLB)) calculations were based on medicare fee-for-service beneficiaries ( $N=21,624,228$; age>68 years), of whom 3,110,654 had dementia [Goodman et al., 2017]. MDD subtypes were derived from patients enrolled in the NIMH Sequenced Treatment Alternatives to Relieve Depression (STAR*D) trial (ages 18-71 years), with sex-specific subtype analysis derived from 952 males and 1589 females with MDD [Marcus et al., 2008]. BD I and II were not available by sex in the GBD, so we 
present the annual first incidence rate of BD I and II in males and females from a US sample $(\mathrm{N}=34,653)$ [Grant et al., 2009]. MS subtypes were derived from the MSBase registry sample (RRMS N =6452; SPMS $\mathrm{N}=594$; RPMS $\mathrm{N}=303$, PPMS $\mathrm{N}=881$ ) collected between the years 1951-2012 across 25 countries and 55 MS centers [Kalincik et al., 2013]. Collectively, these data represent the most current large-scale information on sex-specific disease composition. *Weighted sex ratios were not calculated for disease subtype, as the majority of the samples were not derived from the general population. 
Table 1. Key problems and recommendations for addressing sex-based knowledge gaps in neuroimaging

\begin{tabular}{|c|c|}
\hline $\begin{array}{l}\text { 1. Few large studies comparing sex } \\
\text { differences in MRI outcomes in } \\
\text { major brain diseases }\end{array}$ & $\begin{array}{l}\text { - To overcome sample size limitations, leverage big data } \\
\text { through accessible sources (ENIGMA, UK Biobank, ADNI, } \\
\text { PPMI, NIMH data archive, etc.) }\end{array}$ \\
\hline $\begin{array}{l}\text { 2. "Controlling" for sex as a nuisance } \\
\text { covariate. }\end{array}$ & $\begin{array}{l}\text { - Test sex-by-diagnosis interactions and sex-specific effects } \\
\text { when possible. } \\
\text { - Small samples should conduct sex-specific analyses when } \\
\text { underpowered to examine interactions }\end{array}$ \\
\hline $\begin{array}{l}\text { 3. Inconsistent and/or inappropriate } \\
\text { methods to correct for head size }\end{array}$ & $\begin{array}{l}\text { - Move beyond trying to "correct" for head size and consider } \\
\text { it an explanatory marker } \\
\text { - Avoid methods that use ratios of regional volume to ICV or } \\
\text { TBV to minimize issues with allometric scaling. } \\
\text { - For standard statistical methods, model ICV or TBV as a } \\
\text { covariate as long as these variables do not differ across } \\
\text { levels of the predictor variable }\end{array}$ \\
\hline $\begin{array}{l}\text { 4. Variability in inclusion criteria, } \\
\text { covariate selection, and risk factor } \\
\text { modeling }\end{array}$ & $\begin{array}{l}\text { - Pre-register analytic plan and justify covariate use } \\
\text { - Collect lifetime health histories for all participants } \\
\text { - Report all lifetime comorbidities, particularly those that are } \\
\text { current, to appropriately model and interpret sex effects on } \\
\text { neuroimaging } \\
\text { - Attempt to replicate previous findings by modeling the } \\
\text { same set of covariates when possible } \\
\text { - Model sex-dependent risk factors (e.g., depression, } \\
\text { education, WMH, etc.) using data-driven methods and/or } \\
\text { structural equation models to better interpret sex } \\
\text { differences in brain outcomes }\end{array}$ \\
\hline $\begin{array}{l}\text { 5. Lack of dimensional measures } \\
\text { assessing the clinical relevance of } \\
\text { sex differences in neuroimaging }\end{array}$ & $\begin{array}{l}\text { - Map neuroimaging features to clinical phenotypes and } \\
\text { examining sex differences in these associations. Clinical } \\
\text { phenotyping may be achieved by } \\
0 \text { 1) analyzing NIH RDoC domains across highly } \\
\text { comorbid disorders, or } \\
\text { 2) using data-driven clustering methods that include } \\
\text { item scores on self-report measures and } \\
\text { neuropsychological tests as input features. }\end{array}$ \\
\hline 6. Lack of attention to race/ethnicity & $\begin{array}{l}\text { - Sex-by-race or sex-by-race-by-diagnosis interactions } \\
\text { should be tested when possible. } \\
\text { - Rich assessment of psychosocial and environmental } \\
\text { factors that could bias results } \\
\text { - Examine sex-by-race interactions within distinct } \\
\text { socioeconomic strata }\end{array}$ \\
\hline
\end{tabular}

